\title{
NEIGHBORHOOD DISORGANIZATION, SOCIAL SUPPORT, SUBSTANCE USE AND FUNCTIONING AMONGST ADOLESCENTS; AN ANALYSIS OF THE OHIO BEHAVIORAL HEALTH JUVENILE JUSTICE INITIATIVE
}

\author{
by
}

\section{HEATHER MARIE RICE RN, MSN, CPNP-PC, PMHS}

\author{
Submitted in partial fulfillment of the requirements for Doctor of Philosophy \\ Dissertation Advisor: Dr. Carol M. Musil PhD, RN, FAAN, FGSA
}

Frances Payne Bolton School of Nursing

CASE WESTERN RESERVE UNIVERSITY

August 2017 


\title{
CASE WESTERN RESERVE UNIVERSITY SCHOOL OF GRADUATE STUDIES
}

\author{
We hereby approve the dissertation of \\ Heather M. Rice RN, MSN, CPNP-PC, PMHS \\ Candidate for the Doctor of Philosophy degree*
}

Committee Chair

Dr. Carol M. Musil PhD, RN, FAAN, FGSA

Committee Member

Marguerite (Peg) DiMarco PhD, RN, CPNP

Committee Member

Camille Warner PhD

Committee Member

Jeff Kretschmar PhD

Date of Defense

June 5, 2017

*We also certify that written approval has been obtained for any proprietary materials contained therein 


\section{DEDICATION}

This dissertation is dedicated in loving memory of my two guardian angels, Grandmother-Clara M. Barnes \& Godmother-Margaret D. Boling. Two virtuous women whose presence left profound and lasting impressions on my heart and in my life and you are both greatly missed! 


\section{TABLE OF CONTENTS}

$\underline{\text { Page }}$

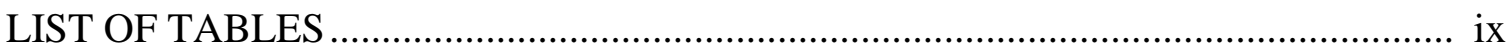

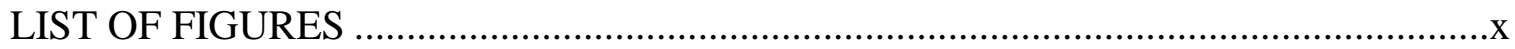

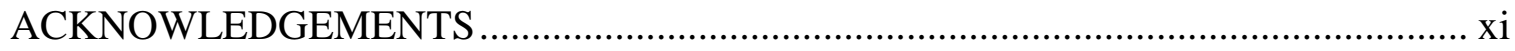

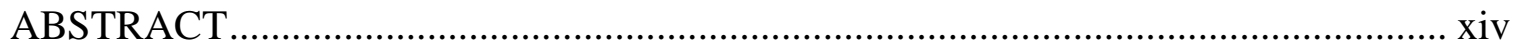

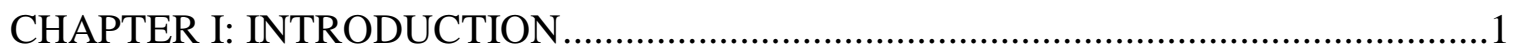

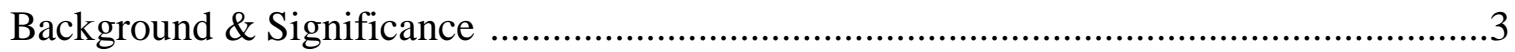

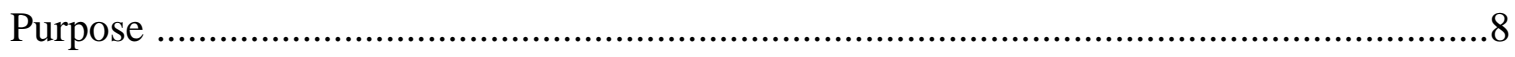

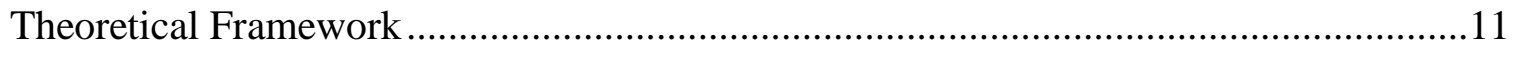

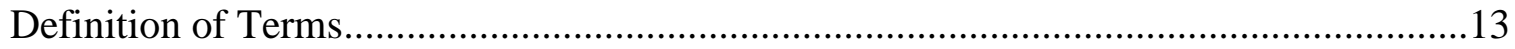

Research Questions ............................................................................................16

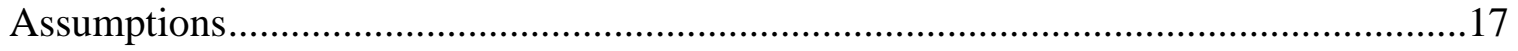

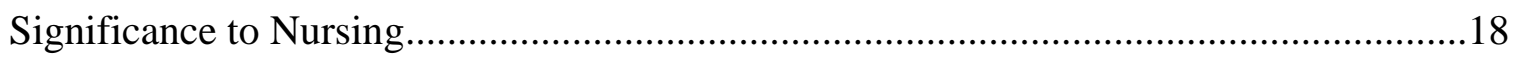

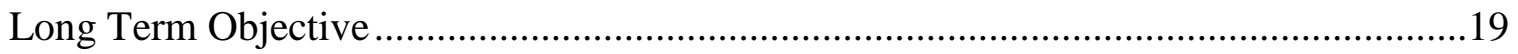

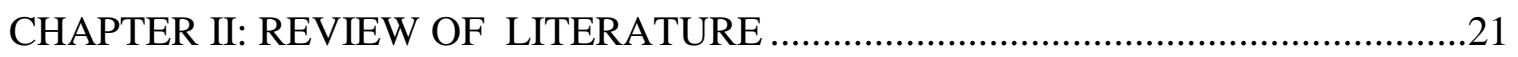

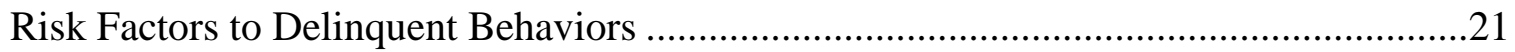

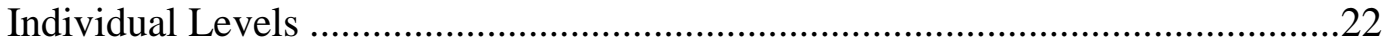

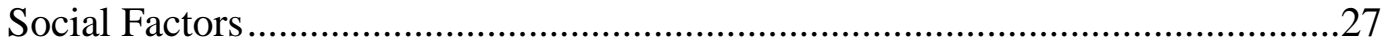

Community Factors ……………………......................................................

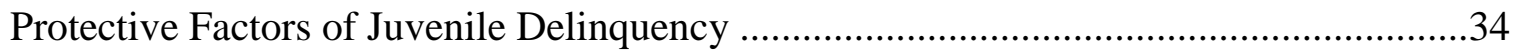

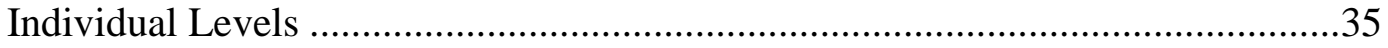

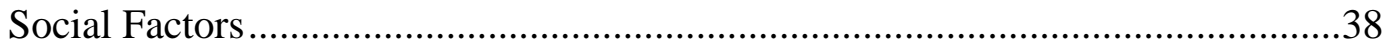

Community Factors........................................................................................... 41

History of Juvenile Delinquency ................................................................................42

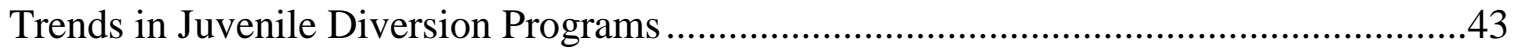


Successful Juvenile Justice Initiatives

Outcomes of the Ohio Behavioral Health Juvenile Justice................................................49

Mental Health and Juvenile Delinquency ……………...............................................50

Depression among Juvenile Offenders …………………………………….....51

Suicidal Ideation among Juvenile Offenders .................................................52

Mental Illness affect on Functioning ...................................................................53

Benefits of Social Support and Mental Illness....................................................54

Adolescent Substance Use (Trends and Relationship to Juvenile Delinquency) ...............55

Substance Use Relationship to Juvenile Delinquency ............................................56

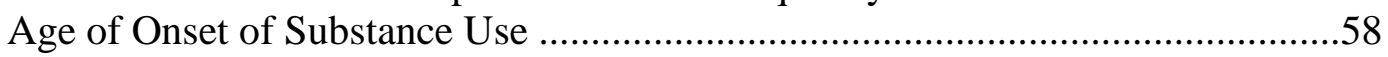

Influence of Neighborhood Conditions on Substance Use ..............................................59

Substance Use Trends (Alcohol, Tobacco and Marijuana) ..............................................61

Alcohol Use among Adolescents (Trends and Effect)....................................................62

Tobacco Use Among Adolescents (Trends and Incidence) ...............................................63

Marijuana Use Among Adolescents (Trends and Incidence) ...........................................64

Substance Use and Depression ...............................................................................66

The Impact of Substance Use on Functioning ................................................................67

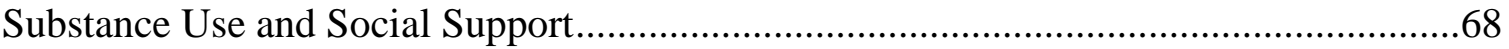

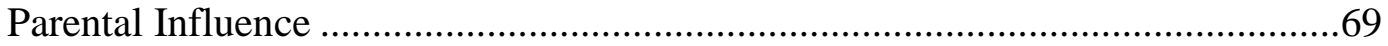

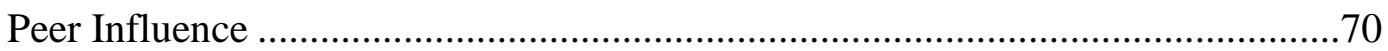

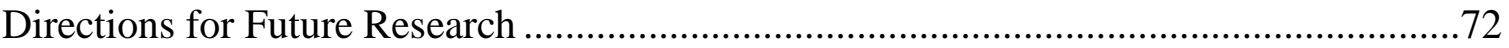

CHAPTER III: RESEARCH DESIGN AND METHODS...............................................74

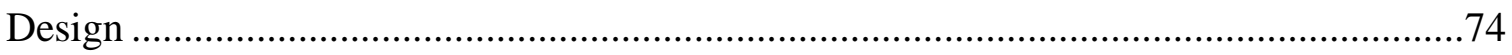

Research Questions ..................................................................................................

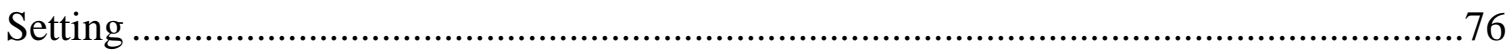

Sample

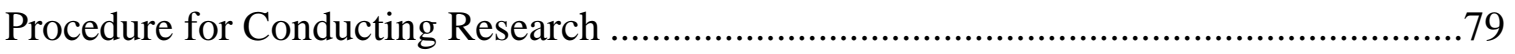




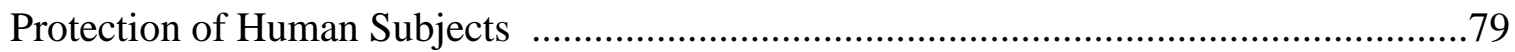

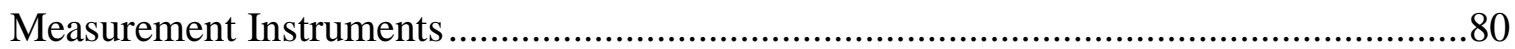

Demographic Information...................................................................... 80

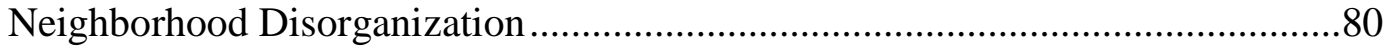

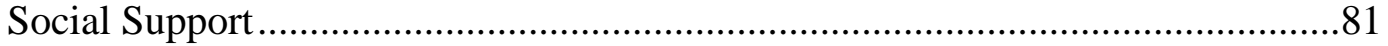

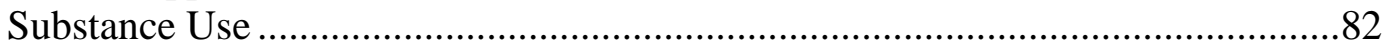

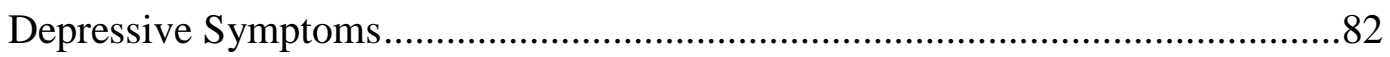

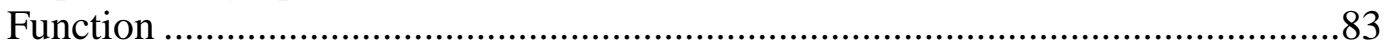

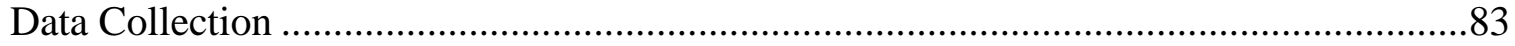

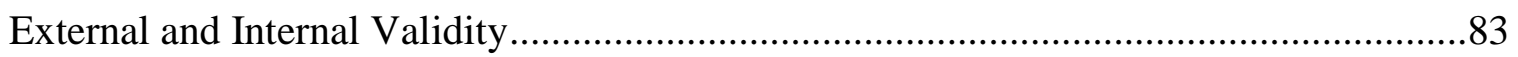

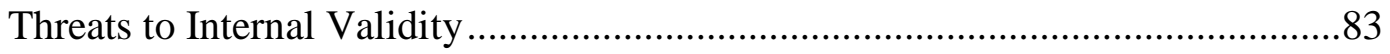

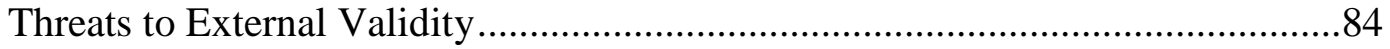

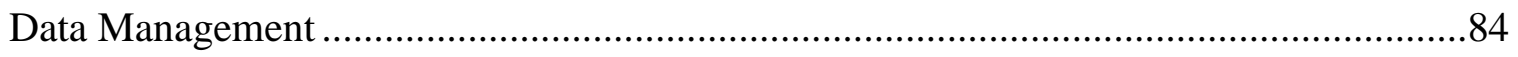

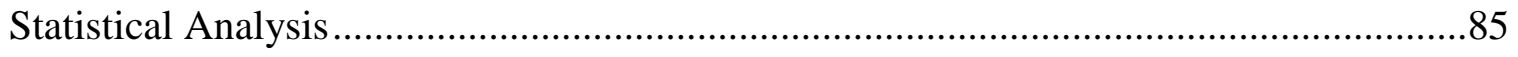

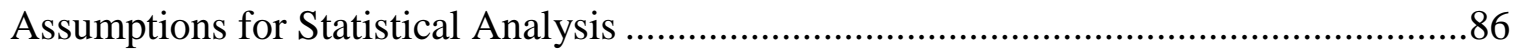

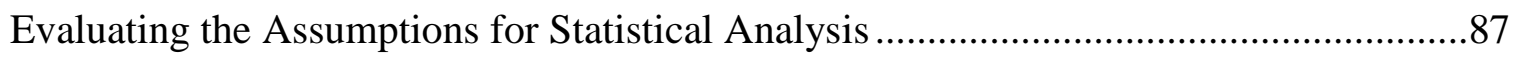

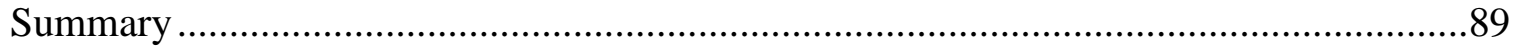

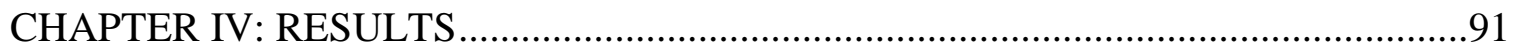

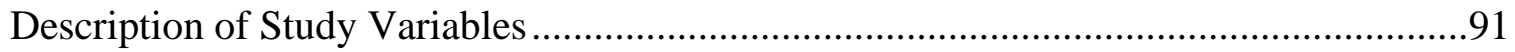

Demographic Characteristics of Study Participants ...............................................92

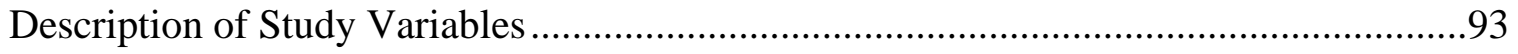

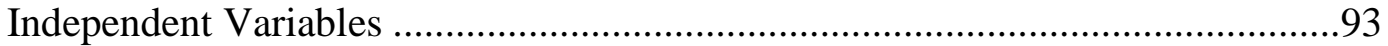

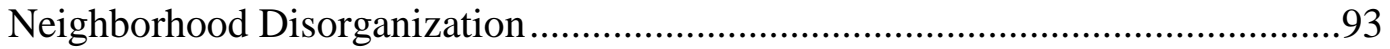

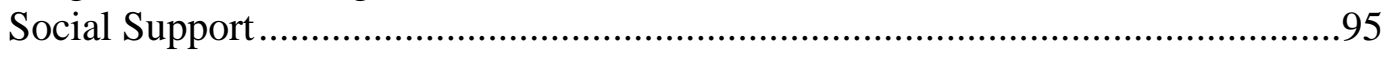

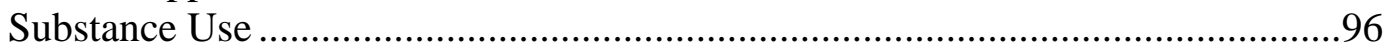

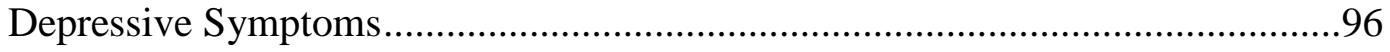

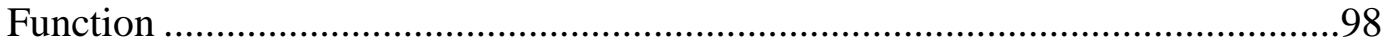

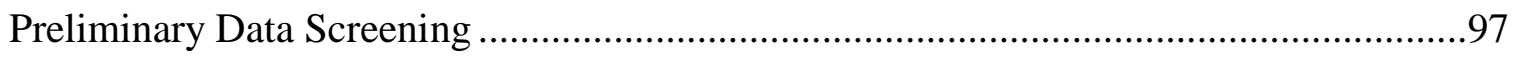

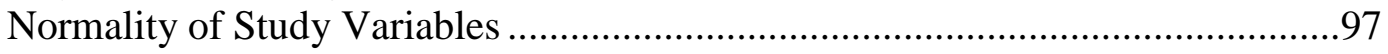

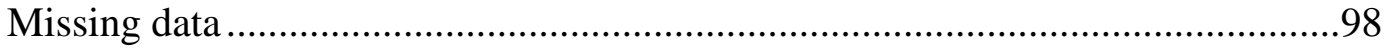

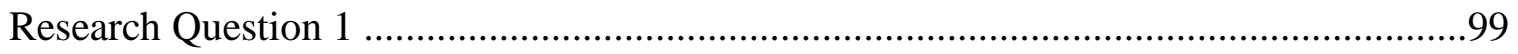




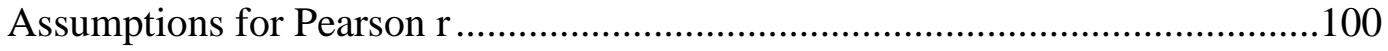

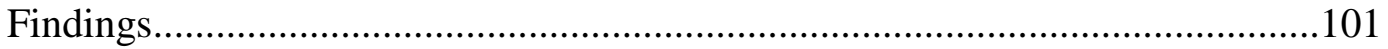

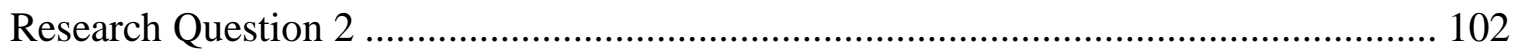

Assumptions for Independent Samples t-test............................................103

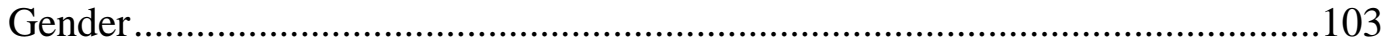

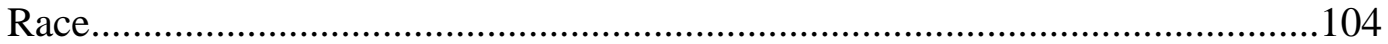

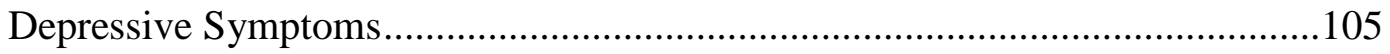

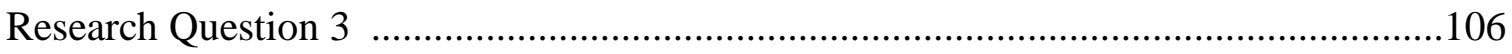

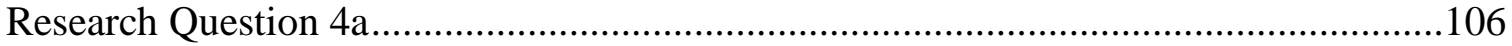

Primary and Secondary Assumptions for Hierarchical Multiple Regression ......108

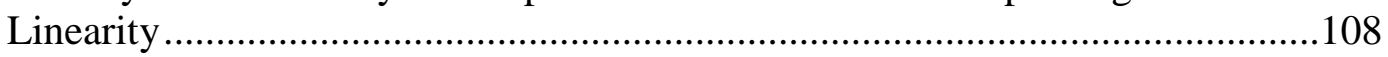

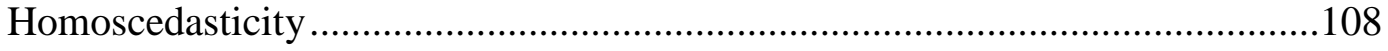

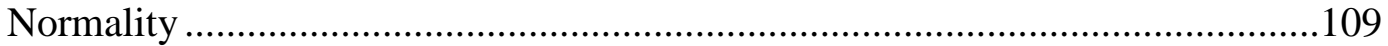

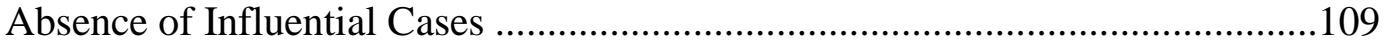

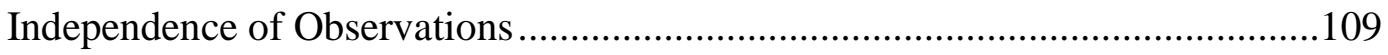

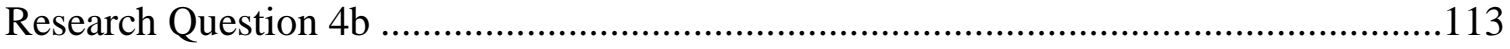

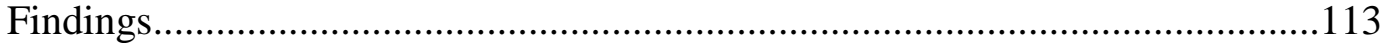

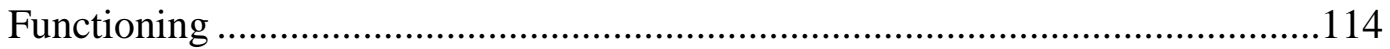

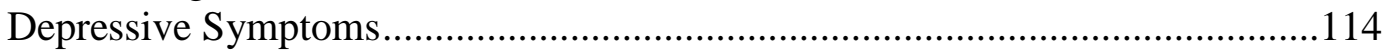

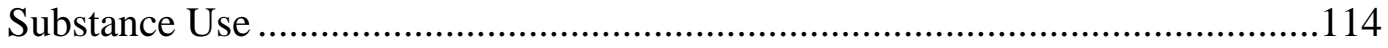

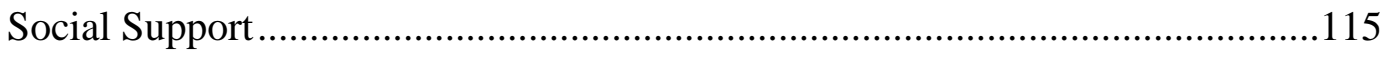

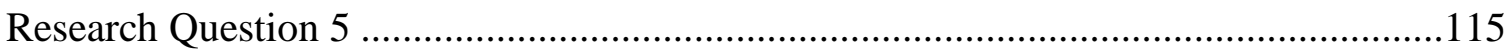

Neighborhood Disorganization and Functioning ...........................................116

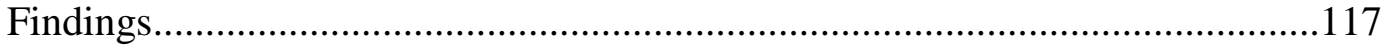

Neighborhood Disorganization and Substance Use......................................117

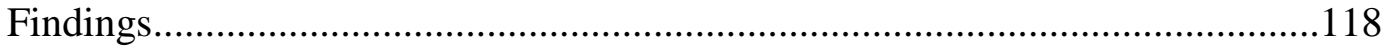

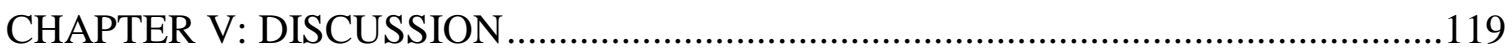

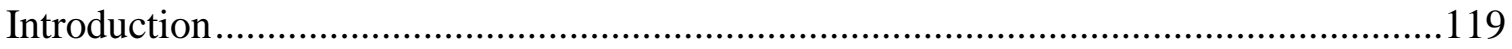

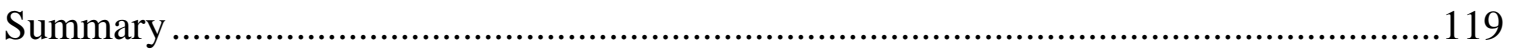

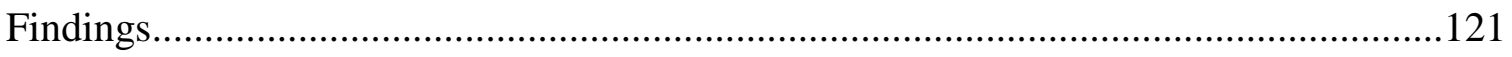

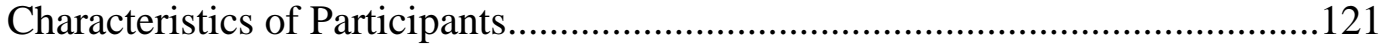

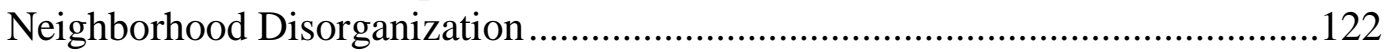

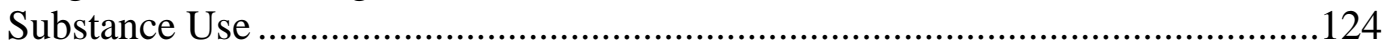

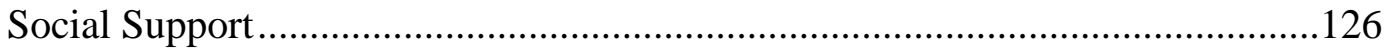

Depressive Symptoms .............................................................................129

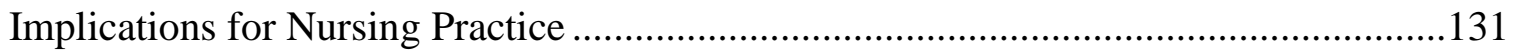




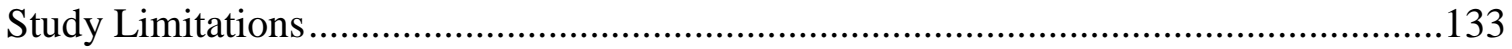

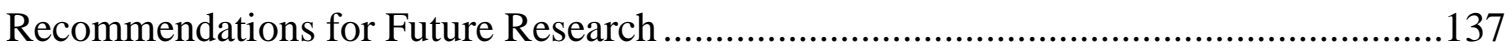

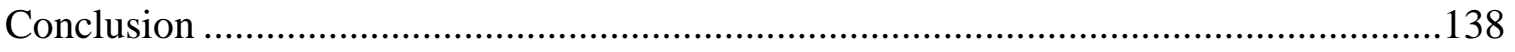

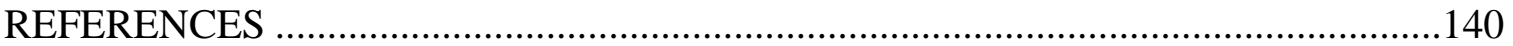




\section{LIST OF TABLES}

Table 1. Protocol Implemented by County ….......................................................77

Table 2. Youth Location by County ................................................................... 92

Table 3. Descriptive Statistics: Individual Characteristics of the Youth $(\mathrm{N}=408) \ldots \ldots \ldots . .93$

Table 4. Normality of Independent and Dependent Variables ...................................98

Table 5. Descriptive Statistic- Age of Alcohol, Tobacco and Marijuana Drug Onset ......99

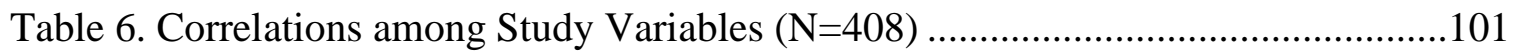

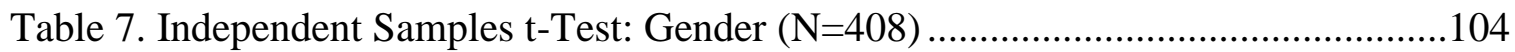

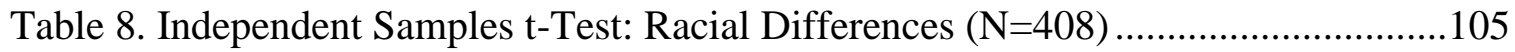

Table 9. Independent Samples t-Test: Depressive Symptoms $(\mathrm{N}=384)$........................106

Table 10. Independent Samples t-Test: Age of First Substance Use ............................107

Table 11. Multiple Regression Zero Order Correlations ..........................................111

Table 12. Summary of Hierarchical Regression Analysis for Functioning ( $N=408)$......112

Table 13. Test of Support as Mediator between ND and Functioning ..........................116

Table 14. Test of Support as Mediator between ND and Substance Use ......................118

Note.

$\mathrm{ND}=$ Neighborhood Disorganization 


\section{LIST OF FIGURES}

Figure 1. Mediation Model (Neighborhood Disorganization and Functioning) .................9

Figure 2. Mediation Model (Neighborhood Disorganization and Substance Use).............9

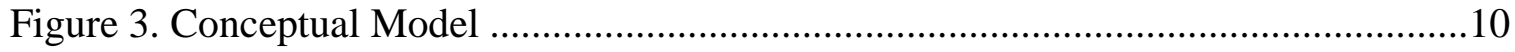

Figure 4. Theoretical Substruction.....................................................................

Figure 5. Derived Bronfenbrenner Ecological Model for Study Variables ......................12

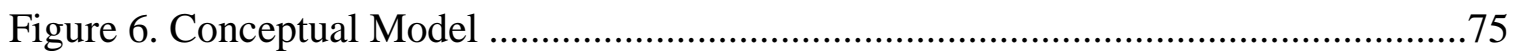

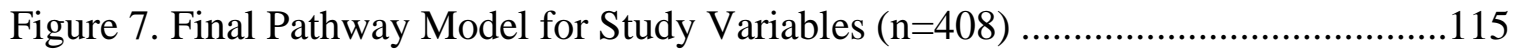




\section{ACKNOWLEDGEMENTS}

"Press on, endure and remain fixed on the victory which lies ahead, your hardwork is not in vain and the reward will make it all worthwhile..."

First, giving honor to my Lord and Savior Jesus Christ, who is the head of my life for without him none of this would have been possible. Thank you for ordering my steps and giving me the strength and courage to pursue this degree and see it to completion. All that I am and ever hope to be, I owe it all to thee!

Immeasurable appreciation and gratitude is extended to the Frances Payne Bolton School of Nursing Legacy Fellowship program and the American Association of College of Nursing (AACN) Jonas Nurse Leaders Scholar program for providing me with the financial means to support my doctoral degree. Your kindness and generosity was an invaluable contribution to the completion of this doctoral program.

I would like to extend my sincere gratitude and appreciation to my committee for assisting in my academic endeavors and professional development. To my dissertation chair Dr. Carol Musil, thank you for agreeing to be my advisor at a crucial time in my doctoral journey and guiding me skillfully and respectfully all the way to the end. From the first semester of my $\mathrm{PhD}$ program you have always expected me to strive for excellence and I can say confidently this dissertation is a reflection of my best work. To Dr. Dimarco and Dr. Warner thank you for your willingness to serve as members on my committee. Your warm encouragement and thoughtful guidance was a critical contribution to the completion of this dissertation. To Dr. Kretschmar, I am forever grateful to you and the Begun Center for Violence Prevention Research and Education for allowing me an opportunity to access your Behavioral Health Juvenile Justice (BHJJ) data and research critical concepts that coincide with my clinical interests. Your support 
and guidance through this process has been instrumental in helping me to articulate new perspectives related to risks behaviors, mental health and functioning in this population.

To my mentor Dr. Melissa Pinto thank you for providing me with the research opportunity that further ignited my passion for adolescent mental health and encouraged me to pursue a doctoral degree. You have taught me so much about the field of research and you always motivate me to press forward in spite of the challenges that may come my way. I appreciate that in spite of the distance you have always been one call away. Thank you for always believing in me, I cherish the person that you have become in my life.

I also want to thank all of the professors and staff who have made this experience at Frances Payne Bolton School of Nursing enjoyable. This University is a special place where people are able to learn in unique and exciting ways that encourages them to think beyond the impossible and I'm honored to have been a student at this University. A special thank you is extended to my fellow $\mathrm{PhD}$ classmates who embarked upon this journey with me. I am so proud to know each of you and I wish you nothing but the best in your future endeavors. To my dear friend Sarah Miano, thank you for helping me to "keep it together" and consistently reminding me that there is a light at the end of this tunnel. I look forward to seeing the impact that the "Dr. Pea Pods" will have on adolescent research.

I would also like to acknowledge my loved ones, "faithful friends", colleagues at the Centers for Families and Children, Houston family (Cynthia \& Chris Evans), church members at East View United Church of Christ and loyal sisters of Delta Sigma Theta Sorority Incorporated (Greater Cleveland Alumnae Chapter). Thank you for praying for 
me, offering words of encouragement and inquiring about my progress. It is a true blessing to be cared about by so many and I thank God for placing people in my life who have always been supportive of my goals. May God continue to bless each of you abundantly!

Last by not least, I want to thank my "parentals", Reverend Mack and Rita Rice, for their unconditional love, support and understanding over the years. I cannot express how grateful I am for the sacrifices that you have made to help me achieve my dreams. Thank you for always believing in me and inspiring me to pursue my passions with unwavering tenacity. I would not be the woman I am today had it not been for both of you. Your love, support and encouragement means more to me than any degree I have obtained to date. I hope that I have made you proud, because this one is for you!

My educational journey has been long and arduous met with moments of extreme elation and periods of fatigue and frustration. I never would have anticipated in the midst of such a demanding process that I would endure an unforeseen circumstance that compromised my health and would potentially threaten my ability to complete this doctoral degree. But God has provided me with the strength to endure in spite of the circumstances and I am thankful for his grace, favor and faithfulness. I appreciate everything that I have learned along this journey and I hope that my dissertation findings will be a useful contribution to such a critical research area. I commit to using my $\mathrm{PhD}$ to fulfill my purpose and destiny in this life. For me the doctorate degree is not the ultimate goal but the vehicle that will drive my movement in clinical research and practice and the best is yet to come!

To God Be The Glory! 
Neighborhood Disorganization, Social Support, Substance Use and Functioning amongst Adolescents; An Analysis of the Ohio Behavioral Health Juvenile Justice Initiative Abstract

by

\section{HEATHER M. RICE RN, MSN, CPNP-PC, PMHS}

Juvenile delinquency is a serious public health concern that places adolescents at risk for poor academic performance, violence, aggression and teen pregnancy. Approximately $70 \%$ of juveniles in the United States legal system also struggle with some form of mental health or substance abuse disorder and most juvenile courts are illequipped to manage these adolescent's complex needs. Diversion programs provide an alternative to the formal court processing and redirect offenders through programming, supervision and intensive family therapy. The purpose of this study was to develop a path model examining the relationships and identifying whether neighborhood disorganization, social support, substance use and depressive symptoms were predictors of functioning in a sample of adolescents involved in the juvenile justice system. The study also determined whether social support mediated the relationship between neighborhood disorganization and functioning and neighborhood disorganization and substance use. This project is guided by Bronfenbrenner's Ecological Transactional Theory of Human Behavior and focuses on key factors nested within the microsystem (functioning, substance use), mesosystem (social support) and exosystem (neighborhood disorganization). This descriptive correlational study is a secondary analysis of the Ohio Behavioral Health Juvenile Justice Initiative (BHJJ), a diversion program for 10-18 year old delinquents with mental health issues and behavioral problems. The subsample 
( $\mathrm{n}=408)$ was comprised of Ohio youth who completed the Youth Information Questionnaire (YIQ) at intake between 2006-2007. The sample was primarily white, male with a mean age of 15 years old. Measures included: Substance-Use Survey-Revised (ever trying tobacco, alcohol or marijuana), Youth Information Questionnaire (social support), Ohio Youth Problem, Functioning and Satisfaction Scale (functional capacity), Trauma Symptom Checklist for Children (depressive symptoms) and neighborhood disorganization (levels of neighborhood disorder at the zip code level). Parental social support was the strongest predictor of functioning followed by race, age and ever-trying marijuana. Parental and peer social support were not found to be mediators between neighborhood disorganization and functioning and neighborhood disorganization and substance use. Nurses have a unique opportunity to provide comprehensive assessments that identify these youth early, provide health education and evidence-based interventions that encourage parental engagement and social support. 


\section{Chapter I}

\section{Introduction}

Juvenile delinquency in the nation's cities, suburbs and rural communities has been considered a long-standing problem with severe implications for not only youth offenders but their families and communities as well. Juvenile crime is indisputably a serious social issue that puts youth at risk for drug use and dependency, school drop-out, incarceration, violence and teen pregnancy (Hoeve, McReynold \& Wasserman, 2014; Greenwood, 2008). In 2013, juvenile courts in the United States handled more than one million delinquency cases that involved juveniles charged with criminal law violations (Hockenberry \& Puzzanhera, 2014; www.bjs.gov). Delinquency offenses are acts committed by juveniles that if committed by an adult could result in criminal prosecution (Hockenberry \& Puzzanhera, 2014). According to the Bureau of Justice Statistics (2012) juvenile offenses involved burglary, larceny-theft, arson, motor vehicle theft, assault, and drug abuse violations (www.bjs.gov). On average over the course of the last 25 years, juveniles were involved in $25 \%$ of serious violent victimizations that includes rape, robbery, aggravated and simple assault (www.bjs.gov).

The problem of juvenile delinquency has been addressed by a variety of stakeholders including law enforcement agencies, community organizations and school systems with the purpose of diminishing the problem. Although, there are societal and individual factors that increase the likelihood of delinquency, partaking in opportunities that effectively address familial, community, developmental and individual factors with effective juvenile justice programming is one operative approach to reduce unwanted behaviors (Hockenberry \& Puzzanhera, 2014; Greenwood, 2008). The current climate of juvenile justice reform is shifting towards prevention and intervention, rather than 
complete suppression by way of detainment (Hockenberry \& Puzzanhera, 2014).

Strategies and program models have been identified that reduce delinquency and promote pro-social development and improve functioning (Hockenberry \& Puzzanhera, 2014;

Greenwood, 2008). Addressing juvenile delinquency prevents the onset of adult criminal activity and thus reduces the burden of crime on its victims and society (Greenwood, 2008; Skowyra \& Powell, 2006). Juvenile delinquency is also a financial burden; it costs billions of dollars to arrest, prosecute, incarcerate and treat juvenile offenders (Greenwood, 2008). Delinquency prevention programs save taxpayers 7 to 10 dollars for every dollar invested, while simultaneously improving the health outcomes for the delinquent youth (Greenwood, 2008). In addition to this, it restores a sense of self-worth and dignity in the youth offenders encouraging them to be productive members of society and an asset to their community (Hockenberry \& Puzzanhera, 2014; Skowyra \& Powell, 2006).

A juvenile's initial contact with the juvenile justice system in Ohio is usually due to arrest by law enforcement or referral by parents, schools, victims or probation officers. Ohio's juvenile court filings as well as the juvenile population have steadily decreased since 1980 from 29\% to 24\% (Children's Defense Fund, 2010). In 2009, 29\% of crimes committed by juveniles in Ohio were property crimes, $22 \%$ were for crimes against people, $5 \%$ were unruly offenses and $44 \%$ were classified under "other offenses" (http://www.publicsafety.ohio.gov). Ohio trends are similar to national rates with between $50-70 \%$ of youth who appear in court having a serious mental health and/or substance use problems (Kretschmar et al., 2014; Skowyra \& Powell, 2006). Recognizing the need to have resources and expertise in the area of early identification, assessment 
and treatment, the state allocated funding towards the development of the Behavioral Health Juvenile Justice (BHJJ) initiative (Kretschmar et al., 2014; Cocozza \& Skowyra, 2000). The BHJJ is a diversion program for youth between the ages of 10-18 involved in the juvenile justice system in Ohio who also suffer from mental or behavioral health issues (Kretschmar, Butcher \& Flannery, 2013). This program was initiated in 2005, represents 17 counties, has serviced well over 3,500 youth and is supported by the Department of Mental Health and Addiction Services and the Ohio Department of Youth Services. The intent of the initiative was to improve behavioral health screening and assessment of youth in the juvenile justice system (Kretschmar et al., 2014). The program is geared towards offenders $10-18$ years old with mental health issues and substantial behavioral, cognitive and emotional problems. The program diverts these youth with mental health or substance use issues from detention into intensive community and evidence-based treatment (Kretschmar et al., 2014).

\section{Background \& Significance}

Adolescence is defined as the transition phase between late childhood and adulthood (Konrad, Firk \& Uhlhaas, 2013). The onset of adolescence is characterized by the initiation of pubertal maturation in response to a rhythmic release of gonadotrophinreleasing hormone $(\mathrm{GnRH})$ from the hypothalamus (Crone \& Dahl, 2012; Blakemore, Burnett \& Dahl, 2010). This process usually begins between ages 8 and 14 years in females (mean age 11), and between ages 9 and 15 in males (mean age 12) (Crone \& Dahl, 2012). Adolescents experience numerous changes in social, academic and other environmental influences thus entering a stage of psychological transition. Adolescence is recognized as a sensitive life phase where key public health and social issues present 
themselves or escalate (Sawyer et al., 2012; Viner et al., 2012). Part of this sensitivity is largely a result of the social embedding of health risk that is more commonly found amongst this age group (Sawyer et al., 2012). Developmental affects related to puberty and rapid brain maturation leads to the development of persistent patterns of lifestyle and health behaviors (Sawyer et al., 2012; Viner et al., 2012). Puberty also represents a period of profound transition in terms of drives, emotions, motivations and social life.

Encouraging the formation of positive health habits early may improve the long-term health and well being of adolescents as they mature into adulthood.

During this developmental stage, adolescents learn how to establish and nurture relationships, develop independence and identity and foster self-confidence, self-control and social skills (Crone \& Dahl, 2012). Environmental factors such as family, peers, school and neighborhood can either support or challenge a young person's overall health and functioning (Viner et al., 2012). Adolescents who identify having someone to care for them and provide assistance are buffered against adverse life events and thus enhance their quality of living (Cutrona, 2000). Social support plays a key role in enhancing mental health and promotes positive coping strategies, particularly in times of distress and crisis.

Social context is a salient influence on adolescent decision-making. Peer influence operates through two processes: social comparison and social conformity (Reppucci, 1999). Adolescents use their peer's actions as a measure of their own behavior. A study by Gardner \& Steinberg (2005) found that peer observation resulted in greater activation in the reward related regions such as the ventral striatum and orbitofrontal cortex. An adolescent's desire for peer acceptance and approval may also 
lead to poor decision making behaviors. In a study conducted by Blakemore \& Robbins (2012), adolescents (ages 13-16), youth (ages 18-22) and adults (ages 24 and over) took the same number of driving risks when driving alone, whereas adolescents (ages 13-16) took almost three times the number of risks when their peers were present. Their lack of experience and exposure causes them to misinterpret and miscalculate the magnitude of the risks and potential harm (Blakemore \& Robbins, 2012; Galvan et al., 2007; Reppucci, 1999).

In addition to the adolescent's social support network, the community that they reside in can also influence their propensity to engage in risky behaviors. Neighborhood disorganization is a concept derived from the social disorganization theory that focuses on the relationship between neighborhood structure, social control and crime (Vakalahi, 2001, Petraitis, Flay \& Miller, 1995). This theory suggests that an individual's residential location is a substantial factor shaping the likelihood that someone will become involved in delinquent behaviors (Vakalahi, 2001, Petraitis, Flay \& Miller, 1995). Adolescents that reside in disorganized neighborhoods that are characterized by poverty, violence and substance use are at risk for a variety of negative outcomes that includes poor mental health, substance use and delinquency.

Neighborhoods define the quality of schools, available transportation, employment opportunities and access to healthcare services. Social and economic features of neighborhoods have been linked with mortality, morbidity, disability, birth outcomes, chronic disorders, health behaviors, mental health, violence exposure and other vital health indicators (Sampson, Morenoff \& Gannon-Rowley, 2002; Pickett \& Pearl, 2001; Yen \& Syme, 1999). Neighborhood disorganization is the combination of low 
economic status, ethnic heterogeneity, residential mobility, family disruption, and urbanization that can contribute to the prevalence of violent or delinquent behaviors (Sampson \& Groves, 1989). Measure of neighborhood disadvantage, family disruption, residential mobility and urbanization weakens the ability of local residents to achieve mutual goals and resolve conflict (Osgood \& Chamber, 2000).

The prevalence of anti-social and delinquent behavior in juveniles has increased dramatically over the past decade, along with the prevalence of mental illness and health endangering behaviors such as alcohol, tobacco and other drug use (Hoeve, McReynold \& Wasserman, 2014). The most pervasive and prominent factor found with current research in relation to delinquency is that of existing mental illness and substance abuse issues (Hoeve et al., 2014; Greenwood, 2008; Skowyra \& Cocozza, 2006). It is estimated that approximately $70 \%$ of juveniles in the United States legal system struggle with some form of mental health or substance abuse disorder (Skowyra \& Cocozza, 2006; Kessler, 2002; Goldstrom et al., 2000). The juvenile justice system is often the first time a youth is screened for behavioral health problems, and a majority of systems are ill-prepared to adequately address the mental health needs of these youth (Cocozza \& Skowyra, 2000; Skowyra \& Powell, 2006). Goldstrom et al. (2000) found that $71 \%$ of juvenile detention centers offer behavioral health screenings while only $56 \%$ conducted a full mental health evaluation. The premise of juvenile justice reform is to prevent juvenile delinquency by identifying youth with mental health needs in the legal system and linking these youth to appropriate mental health services. Many communities have developed diversion programs that conduct in-depth assessment and evaluation and provide more comprehensive evidence-based treatment and supervision services. 
Substance use is a public health concern that has gained worldwide recognition. Use of alcohol, tobacco and illicit drugs by adolescents is a major concern due to associations between substance use and adverse developmental outcomes. The National Survey on Drug Use and Health (NSDUH) assessed the prevalence of substance use disorders and found that in 2013, an estimated 21.6 million adolescents aged 12 or older were classified with substance dependence or abuse in the past year, which is approximately $8.2 \%$ of the population, aged 12 or older. Adolescent substance use can lead to an increased risk of involvement in delinquent behaviors and is associated with more depressive symptoms and psychological distress (Monahan et al., 2013; Cleveland et al., 2008; Greydanus \& Patel, 2005). Adolescents who use alcohol, tobacco and other drugs are at risk for having academic difficulties, health related problems, poor peer relationships and involvement with the juvenile justice system (Monahan et al., 2013, Cleveland et al., 2008, Greydanus \& Patel, 2005, Petraitis, Flay \& Miller, 1995).

There has been growing interest to better understand the neural changes that occur during adolescence to shed light on what influences high impact risk behaviors. Risky decisions made during adolescence can have serious implications; the leading cause of death among this age group is accidents, which is commonly the result of risky behaviors and experimentation (Blakemore \& Robbins, 2012; Crone \& Dahl, 2012). The anatomical reorganization of the adolescent neural circuitry leads to profound emotional and cognitive changes (Konrad, Firk, \& Uhlhaas, 2013). This imbalance caused by the early maturation of subcortical brain areas and the delayed maturation of prefrontal control areas results in a system heavily influenced by rewards and emotions rather than rational decision-making capacity (Konrad et al., 2013, Chein et al., 2011: Galvan et al., 2007). 
Developmental neuroscientists suggest that adolescents may also be more vulnerable to risk-taking because of the limitations in the prefrontal regulatory system to regulate heightened reward and loss signals (Luna, Paulsen, Padmanabhan \& Geier, 2013; Geier \& Luna, 2009). Developmental fMRI studies suggest that adolescents exhibit different responses to rewards due to the hypersensitivity of reward processing regions such as the ventral striatum and orbitofrontal cortex as well as the immature cognitive control ability (Luna et al., 2013; Gladwin et al., 2011; Geier \& Luna, 2009; Galvan et al., 2007).

\section{Purpose}

The purpose of this study was to examine the relationships amongst neighborhood disorganization, social support, substance use (age of first use, type), depressive symptoms and functioning in a sample of adolescents involved in the Ohio BHJJ. This project assessed whether there were differences between neighborhood disorganization, social support and functioning and whether these differences varied by personal characteristics (gender and race) and depressive symptoms. The study also examined whether social support mediated the relationship between neighborhood disorganization and functioning (Figure 1) and neighborhood disorganization and substance use including type of substances ever used including alcohol, tobacco and marijuana (Figure 2). In addition to whether social support mediated the relationship between neighborhood disorganization and functioning, the study also examined whether there were differences in social support and functioning based on age of first substance use being before or after the age of 12. Ultimately, the study developed a model identifying whether neighborhood disorganization, social support, substance use (alcohol, tobacco and marijuana) or depressive symptoms are predictors of functioning and which risk factors conferred the 
greatest risk. Figure 3. illustrates the conceptual model that will guide the research study.

Figure 4 illustrates the theoretical substruction model of the constructs, concepts and level of measurement for each variable.

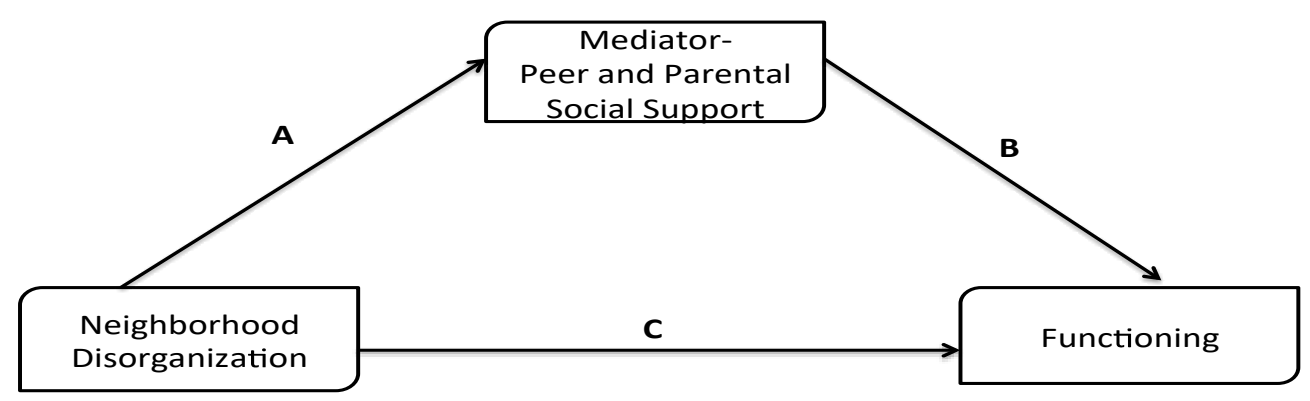

Figure 1. Mediation Model (Neighborhood Disorganization and Functioning)

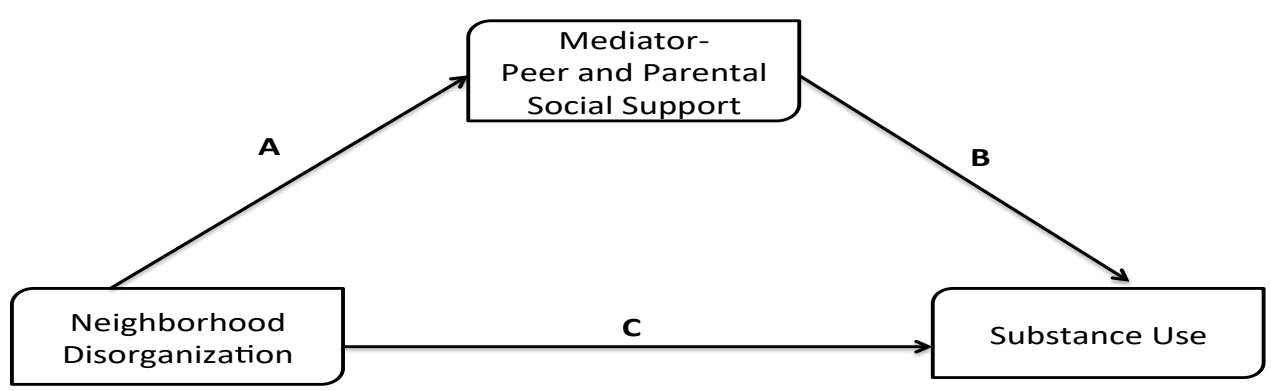

Figure 2. Mediation Model (Neighborhood Disorganization and Substance Use) 


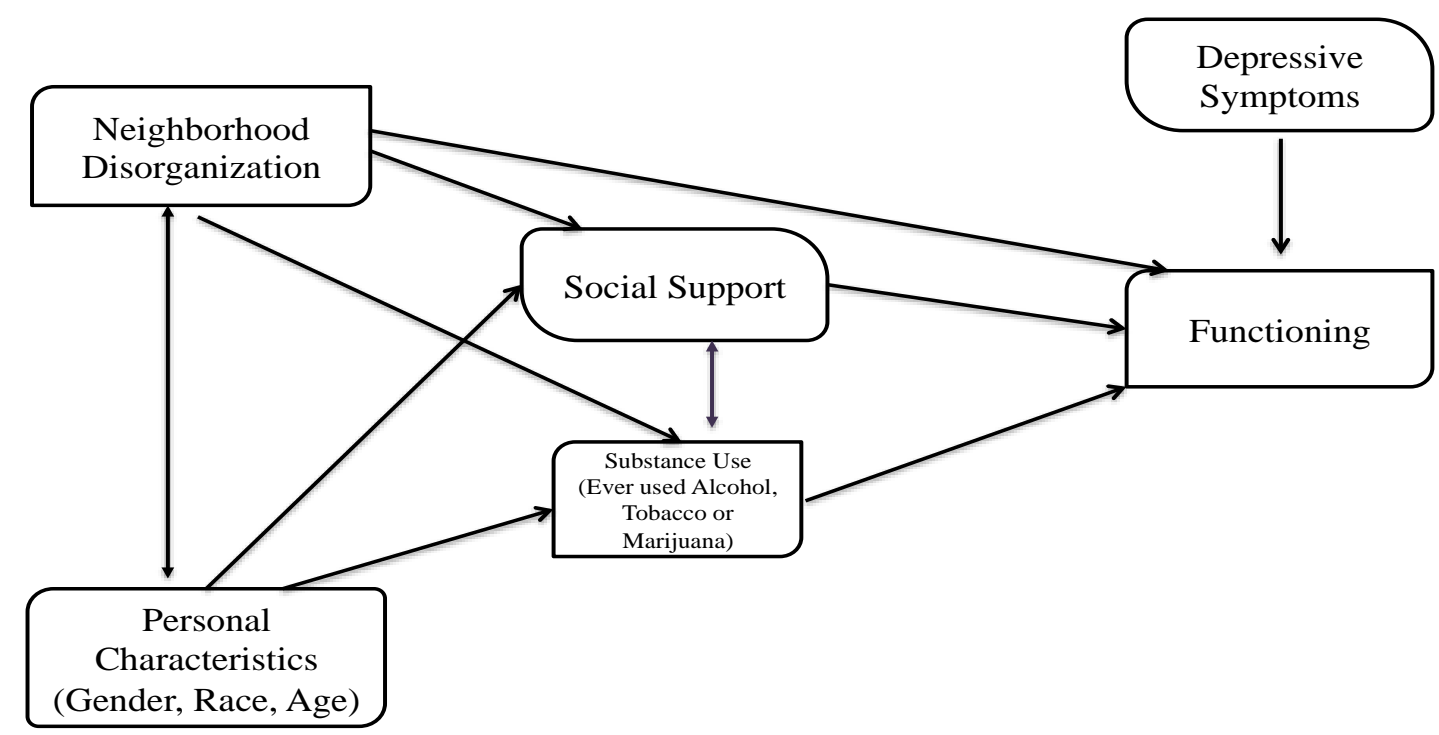

Figure 3. Conceptual Model

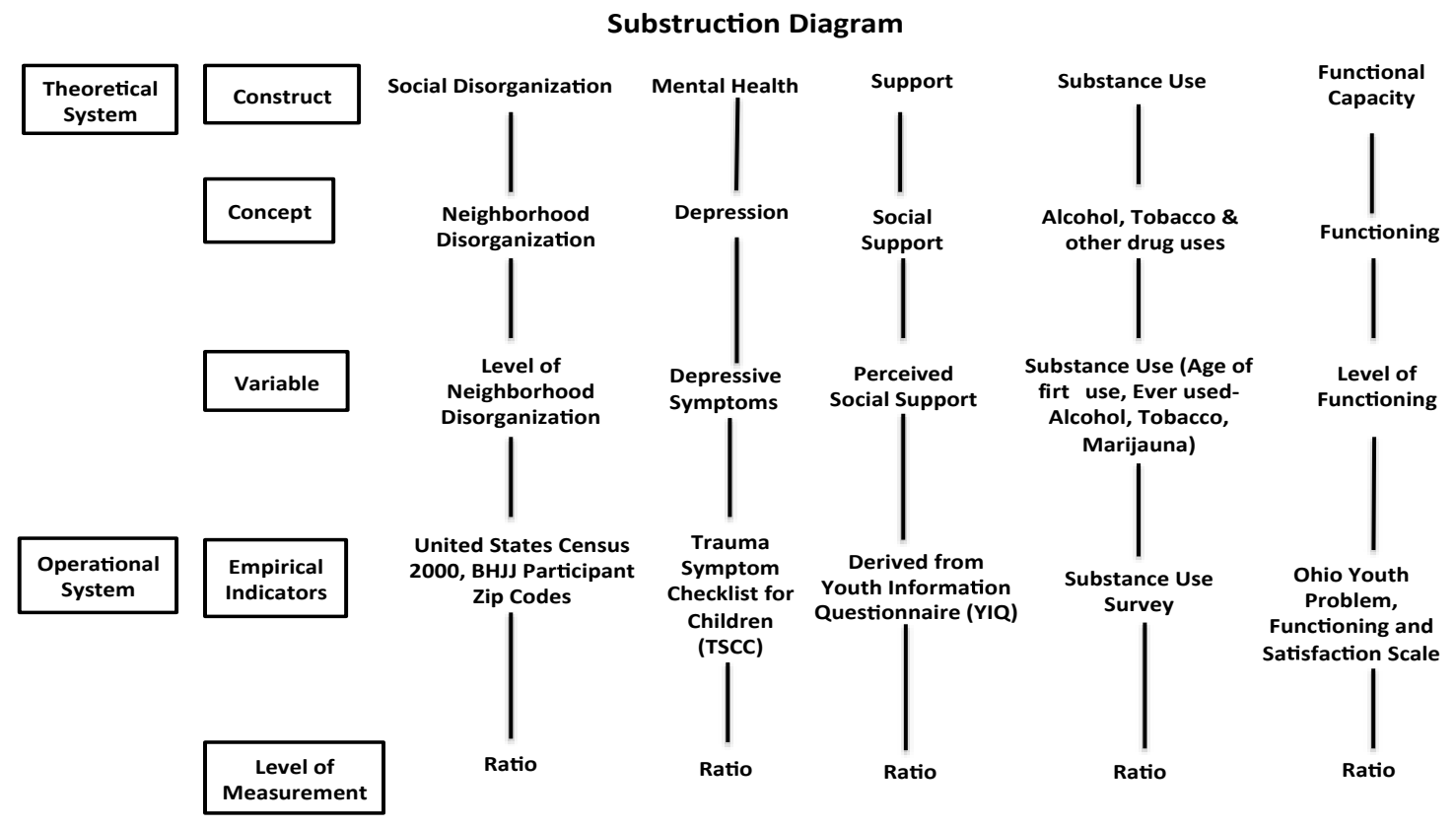

Figure 4. Theoretical Substruction 


\section{Theoretical Framework}

The Bronfenbrenner Ecological Transactional model is a theory of human behavior that guided the theoretical framework for this proposal. This model proposes that nested contexts interact to influence human development. The model is comprised of four systems; the microsystem, mesosystem, macrosystem and exosystem that each indirectly or directly interact with one another to influence growth and development. The microsystem is the setting within which the individual is behaving at any given moment in his or her life. This system directly affects and influences the adolescent's behavior, social relationships, cognition, and physical skills (Handley, Rogosch, Guild, \& Cicchetti, 2015). This system is comprised of family, friends, school, church as well as the neighborhood in which they live. The mesosystem refers to the interrelation between two systems and describes the bridging that is built between the two settings that an adolescent engages (Bronfenbrenner, 2005). The mesosystem includes relationships with family and well as peers or teachers (mentors). The exosystem is an extension of the mesosystem and may not have a direct influence on the developing adolescent but can have an indirect influence on the adolescent's behavior. This system includes family dynamics such as parental conflict with other siblings or the parent's stress from the work environment. Although this does not directly involve the adolescent it can still have an impact of their development. The last system is the macrosystem and includes the cultural environment in which the adolescent resides. This level includes cultural values, public policy, government and laws. This system influences the nature of interaction within all other levels in the model. (Bronfenbrenner, 2005) 
This study focused on some key factors that are nested within the various systems described in this theory. The adolescents are positioned to interact with various contextual levels such as neighborhood, family, school and peers. Bronfenbrenner's model conceptualizes adolescent's development as a collection of factors that will constitute the adolescent experience (Cox, Burr, Blow, \& Parra Cardona, 2011). The model describes that an individual interacts with different contexts to form and guide their development. This study determined whether neighborhood disorganization, social support, substance use and depressive symptoms have a direct or indirect influence on functioning. This project also focused on each system and specifically tested the interrelationships between the microsystem (functioning, substance use), mesosystem (social support) and exosystem (neighborhood disorganization) (Figure 5).

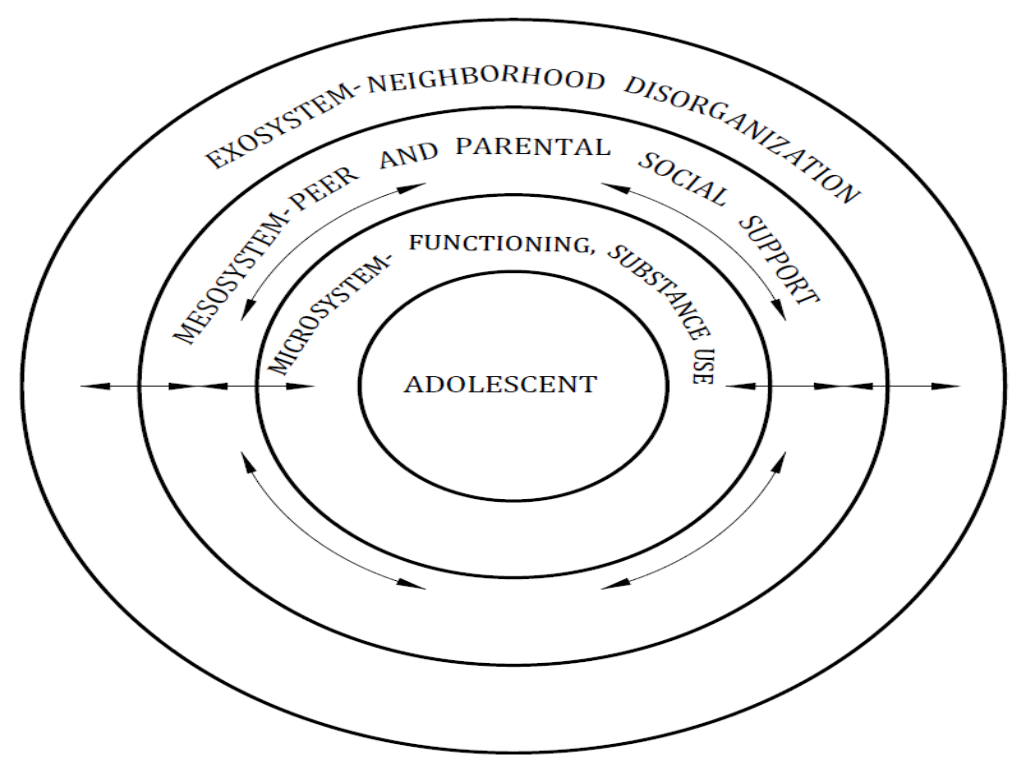

Figure 5. Derived Bronfenbrenner Ecological Model for Study Variables 


\section{Definition of Terms}

Juvenile Delinquency

Juvenile delinquency is defined as participation in illegal behavior by minors (Siegel \&Welsh, 2011). Juvenile delinquents are typically under the age of 17 and commit an act that otherwise would have been charged as a crime if committed by an adult. These acts can include misdemeanors and felonies.

Depression

Depression is a mood disorder characterized by a pervasive and persistent low mood that is accompanied by low self-esteem and loss of interest in pleasure activities (anhedonia) (American Psychiatric Association, 2013; www.nimh.nih.gov). This condition is disabling and adversely affects an individual's appetite, sleep, general health, family and school life. Depressive Symptoms were measured via the Trauma Symptom Checklist for Children (TSCC) and youth were classified as either "depressed" or "nondepressed".

\section{Conceptual and Operational Definitions for Study Variables}

\section{Neighborhood Disorganization}

Conceptual definition- Neighborhood disorganization refers to the observed or perceived physical and social features of neighborhoods that may signal the breakdown of order and social control that can undermine the quality of life (Sampson \& Groves, 1989; Shaw \& McKay, 1942) Social disorder can be exemplified by behaviors such as community violence, substance use, poverty and crime. It's also impacted by gang activity, vacant and abandoned homes, run-down buildings and high levels of police activity also impact it. The concept neighborhood disorganization is made up of seven observed measures that are available from information reported to the U.S Census. This 
includes: percentage of female headed households with children under 18, percentage of residents with less than a high school education, percentage of renter occupied housing, percentage of residents who were unemployed, percentage of residents using supplemental security income, percentage receiving cash public assistance and the percentage of families below the poverty line.

Operational Definition- Neighborhood disorganization was operationalized with the use of the U.S Census at the zip code level. Zip codes were collected for each participating county from all participating adolescents involved in the BHJJ initiative at intake. Each of the seven observed measures were obtained from the 2010 U.S Census as a percentage and converted into a z-score. To reach a final score for neighborhood disorganization Each of the z-scores for the seven observed measures were summed to reach a final level of disorganization. Higher scores indicated greater neighborhood disorder while lower scores indicated that there was less disorder in that area relative to other adolescents in the sample. The z-score method has been validated in research as a valid tool to examine neighborhood disorganization (Dulin-Keita, Casazza, Fernandez, Goran \& Gaver, 2013; Caughy, Nettles \& O’Campo, 2008; Drewnowski, Rehm \& Solet, 2007).

\section{Social Support}

Conceptual definition- Social support is the perception and actuality that one is cared for, has assistance available from other people and is a part of a supportive social network (McGrath, Brennan, Dolan \& Barnett, 2012; Zimmer-Gembeck \& Skinner, 2011; Langford, Bowsher, Maloney \& Lillis, 1997). There are four common functions of social support that includes emotional, tangible, informational, and companionship or intangible support (Cutrona, 2000). Social support can be measured as the perception that one has assistance available or that actual assistance is received (Frison \& Eggermont, 2015; 
Zimmer-Gembeck \& Skinner, 2011; Barrera, 1986). Support is seen as avenues for sharing, attachment, resource allocation, dependence and self-confidence and selfefficacy. Social support includes the degree to which a person is integrated in a social network that can come from various sources including family, friends or adult figures that are involved in the adolescent's life such as a teacher (Frison \& Eggermont, 2015; McGrath, Brennan, Dolan \& Barnett, 2012; Zimmer-Gembeck \& Skinner, 2011). Operational Definition- Social support will be operationalized as one's perception of having an adult or friend present in their life for support or assistance. This form of support is defined as "emotional support" and includes acts of empathy, listening and generally "being there" for someone when needed or in times of distress (McGrath, Brennan, Dolan \& Barnett, 2012; Zimmer-Gembeck \& Skinner, 2011; Cutrona, 2000). This data will be derived from responses provided on the Youth Information Questionnaire (YIQ).

Substance Use

Conceptual definition- Substance use is the use of one or more substances that leads to a clinically significant impairment or distress that is detrimental to one's physical, social, emotional and mental health (American Psychiatric Association, 2013; Pham-Kanter, 2001). Substance use disorders are diagnosed based on the DSM-IV criteria and is characterized by a pattern of continued pathological use that results in repeated adverse social consequences (Pham-Kanter, 2001). This proposal will focus on general substance use focusing specifically on the age of first use and whether someone has ever used alcohol, tobacco and marijuana (American Psychiatric Association, 2013; Pham-Kanter, 2001). 
Operational Definition- Substance use was based on self report from the Substance-Use Survey-Revised a measure adapted from the SAMHSA funded Tapestry Project that collects information regarding frequency of substance use and type of substance used which can include: tobacco, alcohol and other drugs (Kretschmar, Butcher \& Flannery, 2013). This project focused specifically on marijuana use since it's reported as one of the most commonly abused drugs by adolescents and has gained more mainstream attention in response to the push for legalization (www.drugabuse.gov).

\section{Function}

Conceptual definition- Functional capacity is defined as an individual's ability to perform normal daily activities required to meet the basic needs, fulfill specific roles and maintain health and wellness (www.health.gov). Functional status can be influenced by age, biological, mental or physiological impairment as well as one's health perception.

Operational Definition-For this research, functioning will be operationalized based on responses from the Ohio Youth Problem, Functioning and Satisfaction Scale which was designed to assess clinical outcomes for children and adolescents with severe emotional and behavioral disorders (Ogles, Melendez, Davis \& Lunnen, 2001). This scale defines functioning in a variety of daily activity including interpersonal relationships, recreation, self-direction and motivation.

\section{Research Questions}

The purpose of this study will be met by addressing the following research questions: (Q1a-d)- What are the relationships between (a) neighborhood disorganization, (b) social support (peer and parental), (c) substance use (ever using alcohol, tobacco or marijauna) and (d) depressive symptoms and (e) overall functioning in juveniles involved in the behavioral health juvenile justice initiative? 
(Q2a-b)-Are there differences in neighborhood disorganization, social support

and functioning by (a) gender (b) race and (c) depressive symptoms?

(Q3)- Are there difference in social support and functioning by (2) age of first drug use (before or after age 12)?

(Q4a-b) What are the predictors of functioning in youth involved in the Behavioral Health Juvenile Justice Initiative controlling for age, race and gender? What is the final pathway model for the independent and dependent study variables?

(Q5) Does social support mediate the relationship between (a) neighborhood disorganization and substance use (ever using alcohol, tobacco and marijuana) and (b) neighborhood disorganization and functioning?

\section{Assumptions}

The following four assumptions will guide this research study:

(1) Adolescents are in constant, dynamic, reflexive relationship with the environment and respond to that relationship through cognitive processes that are influenced by personal, social, physical and environmental factors. (Tomey \& Alligood, 2002).

(2) Adolescence is a critical developmental period marked with risk taking behaviors that may predispose the adolescent to various stressors that influence their physical, psychological, social and functional health and well being (Luna, Paulsen, Padmanabhan and Geier, 2013; Crone \& Dahl, 2012; Grier \& Luna, 2009).

(3) Adolescents are social beings and through appraisal and coping processes will desire to achieve psychological well-being. This desire for mental stability may be accomplished through individual effort or the guidance and support of family and friends. (Lazarus \& Folkman, 1984) 
(4) Adolescents and caregivers who participated in the Behavioral Health Juvenile Justice diversion program provided valid and honest responses to the questionnaires provided (Kretschmar, Butcher, Flannery \& Singer, 2014; Kretschmar, Butcher \& Flannery, 2013).

\section{Significance to Nursing}

The nursing meta-paradigm includes the following four central concepts: person, health, environment and nursing. This project evaluates a unique subset of adolescents with a mental health diagnosis who are also involved in some capacity with the Ohio juvenile justice system. Some of these youth come from highly disorganized neighborhoods, lack social support and have experimented with alcohol, tobacco or marijuana. These youth are considered at-risk and would benefit from early identification and prompt intervention in order to decrease their chances of enduring long-term adverse health complications.

Health is defined by their physical, social and emotional functioning and is not limited to the absence of disease (Post, 2014). Nurses and advanced practice providers can serve a unique role in identifying youth that may be at risk and perform thorough assessments creating individualized patient treatment plans which addresses their needs. Tailoring the treatment plan to the patient takes into consideration unique contributing factors such as neighborhood conditions, presence or lack of supportive networks and functional capacity.

Environment is recognized as the surroundings or conditions in which adolescents live and operate (Macintyre \& Ellaway, 2003). Environments, particularly disorganized neighborhoods, may influence or predispose an adolescent to risky behaviors through indirect or direct ways. Highly disorganized neighborhoods are plagued with poverty, 
exposure to violence and access to firearms, alcohol and drugs. These environments also lack sufficient access to community resources to foster pro-social adolescent development. Nurses can serve as the liaison to link patients to additional resources or services that are present within the community that can assists them to address their needs.

The American Nurses Association defines nursing as the protection, promotion and optimization of health, prevention of illness and alleviation of suffering through the treatment of human response (www.nursingworld.org). Nurses are a critical component of the healthcare team and have the unique opportunity to address the needs of at-risk youth particularly those who are involved in the juvenile justice system. Nurses are also equipped with the knowledge to provide health education thus improving health literacy amongst these youth and potentially instilling the value of healthy and pro-social behaviors that they can carry on into adulthood.

\section{Long Term Objective}

The long-term objective of this project is to provide a better understanding regarding the relationships amongst variables that may contribute to altered functioning in a group of adolescents involved in the juvenile justice system. These variables specifically address key risks and protective factors that have been identified in this population such as neighborhood disorganization, social support, depressive symptoms and substance use. Having a clearer understanding regarding how these critical factors influence one another can lead to or validate interventions that are designed to prevent adverse health conditions and improve health outcomes among adolescents in the juvenile justice system. In addition to this, the BHJJ initiative has demonstrated much 
success with adolescents that are involved in the juvenile justice system in Ohio and have mental health diagnoses (Kretschmar, Butcher, Flannery \& Singer, 2014; Kretschmar, Butcher \& Flannery, 2013). Over 92\% of caregivers agreed that they were satisfied with the services that their child was receiving through the Behavioral Health Juvenile Justice Program. Nearly 72\% of youth enrolled from 2011-2013 were identified as successful treatment completers and reported an increase in functional capacity and a decrease in problem severity while in the BHJJ program (Kretschmar et al., 2013). These outcomes support the continued funding for diversion programs such as the BHJJ to address the needs of juvenile delinquents who also suffer from a mental health diagnosis. It also provides access to mental health services for adolescents who may not have those resources readily available otherwise. In addition to this, it provides the guardians with additional educational resources, supportive systems and intensive home based counseling that enables them to better address their adolescent's behaviors and individualized needs. 


\section{Chapter II- Review of Literature}

The purpose of this chapter is to summarize and critique the scientific literature related to risk and protective factors to delinquent behaviors, juvenile justice development and an overview of the Ohio Behavioral Health Juvenile Justice Initiative. This chapter will also review the influence of neighborhood conditions, mental health, substance use, social support and functioning in adolescents involved in the juvenile justice system. The empirical literature describing the current state of the science regarding the relationships amongst these variables will also be analyzed and critiqued.

\section{Risk Factors to Delinquent Behaviors}

In the United States, approximately 1 in 8 reported violent crimes are committed by a juvenile offender (FBI, 2012). Incarcerated youth represent a high risk, vulnerable population with disproportionately high rates of physical, emotional, social and mental health needs (Barnert et al., 2015). These youth are at increased risk for reoffending, substance use and adverse health outcomes in adulthood that result in higher mortality. Early and persistent behavioral problems has been linked to poor academic achievement, unemployment and risky health behaviors in adulthood (Bradshaw, Schaeffer, Petras, \& Ialongo, 2010). Therefore, understanding the various causes that may lead to the development of delinquent behaviors is critical in order to better address this public health concern. Each high-risk youth diverted from a life of delinquency saves the government an estimated 2.6-5.3 million dollars in their lifetime (Cohen \& Piquero, 2009). Though a risk factor can increase the probability of offending it does not make offending a certainty. There is no single path that leads to delinquency but the presence of 
several risk factors can increase one's chances of offending. These risk factors can be classified as individual, social and community factors.

\section{Individual Levels}

Psychologists have found that individuals who commit serious violent crimes tend to have experienced higher rates of trauma, maltreatment and abuse in childhood even when controlling for other factors (Fox, Perez, Cass, Baglivio, \& Epps, 2014). Maxfield \& Widom's (1996) study on child abuse found that experiencing trauma and abuse during childhood increases the odds of juvenile violent behavior by more than 200\%. Lansford et al (2007) found that adolescents who had experienced physical abuse in the first five years of life were more likely to have been arrested as a juvenile for violent and nonviolent offenses. In addition to this, African American youth who had been physically abused were twice as likely to have a court record for violent offenses and almost three times as likely to have court records for non-violent offenses. Research also suggests that $90 \%$ of juvenile offenders have experienced a traumatic event during their childhood (Dierkhising et al., 2013). Adverse childhood experiences (ACE's) are categories of commonly experienced trauma and include: emotional abuse, physical abuse, sexual abuse, emotional neglect, physical neglect, witnessing household violence, household substance use, household mental illness and household member incarceration. One method to classify the types of trauma that one experiences is portrayed in a study by Fox et al (2014) that evaluated the impact of (ACE's) on a cohort of juvenile offenders in Florida to better understand the impact that these events have on serious, violent and chronic criminal behaviors. Each ACE reported increased the risk of being a chronic 
offender by more than $35 \%$ even when controlling for known risk factors for delinquent behaviors.

It has long been established that maternal smoking during pregnancy has adverse perinatal consequences that may extend beyond the prenatal period (Wakschlag, Pickett, Cook, Benowitz \& Leventhan, 2002; Olds, 1997). Maternal smoking is a risk factor for conduct disorder, anti-social behaviors and delinquency. Animal studies have established that nicotine crosses the placental barrier and effects neural function and development (Wakschlag et al, 2002; Slotkin, 1998). Moffitt \& Caspi (2001) suggests that neuropsychological impairments disrupt normal in-utero development of verbal and executive functions in the brain. These disruptions have negative effects on learning, reasoning, memory, problem solving, temperament, and impulse control and ultimately increase one's propensity towards delinquent behavior (Murray, Irving, Farrington, Colman \& Bloxsom, 2010; Turner, Hartman \& Bishop, 2007). They also increase ones propensity to exhibit physical aggression and violence towards others (Turner et al., 2007). A study conducted by Murray et al. (2010) identified maternal smoking as a strong predictor of conduct problems and crime emphasizing the effects of toxic nicotine exposure on fetal brain development.

Maternal alcohol use has also been associated with cognitive, behavioral and social deficits. Alcohol is a teratogen that adversely affects central nervous system development (Schonfeld, Mattson \& Riley, 2005). The permanent devastating impact of extreme levels of alcohol exposure in-utero is evidenced in conditions such as fetal alcohol spectrum disorder and fetal alcohol syndrome (Robinson et al., 2010; O’Leary, 2004). Studies suggest that in-utero alcohol exposure has a dose response mechanism 
where light drinking is associated with behavioral dysfunction and heavy drinking is associated with more serious developmental complications (Robinson et al., 2010). There is conflicting research related to the exact amount of alcohol consumption that adversely effects development. A study by Robinson et al (2010) used a longitudinal approach to examine the association of fetal alcohol exposure during pregnancy with child and adolescent behavioral development. The study analyzed 2,370 children exposed to varying amounts of alcohol in-utero and found that low to moderate alcohol intake early in pregnancy was not associated with developmental risks. However, a study conducted by Schonfeld et al (2004) found that participants with prenatal alcohol exposure had a lower level of moral maturity and increased delinquent behaviors compared to the nonexposed control group.

Academic underachievement is a common public health concern that has lasting negative implications for the individual, his or her family and society (Henry, Knight \& Thornberry, 2012). Delinquency is associated with cognitive deficits regardless of sex, race/ethnicity, socioeconomic status, academic achievement and arrest history (Lansing et al., 2014; Moffitt \& Caspi, 2001). Delinquent youth compared to non-delinquent youth may have cognitive deficits related to verbal abilities and perceptual reasoning (Lansing et al., 2014; Moffitt \& Caspi, 2001). A study by Lansing et al (2104) assessed cognitive functioning among participants in an Illinois Juvenile Project and found that for each measure of cognitive functioning including overall intellectual ability, receptive verbal skills and basic academic achievement participants scored below average. The participants exhibited the most deficits in receptive verbal skills, which is the ability to demonstrate one's understanding of a word's meaning without having to describe the 
word. The impact of education on crime rates indicates that there are benefits to education that reduces the propensity to commit a crime (Henry, Knight \& Thornberry, 2012; Weerman, Harland \& van der Laan, 2007). Failure to graduate from high school increases one's likelihood of being involved in criminal behavior (Moretti, 2005). Weerman et al (2007) conducted a longitudinal study in the Netherlands looking at misbehavior conducted with the school and delinquent behavior outside of school. The participants completed a questionnaire that covered 9 misbehaviors at school and 12 types of delinquency outside of school. More than two-thirds of the sample reported at least one of the investigated misbehaviors and almost half reported involvement in delinquent behaviors. The most commonly reported misbehavior and delinquent activities included: fighting without injury, bullying, theft and vandalism. Thus findings revealed a significant relationship between school misconduct in $7^{\text {th }}$ and $9^{\text {th }}$ graders and serious delinquency outside of school one year later. Henry, Knight \& Thornberry (2012) conducted one of the few longitudinal studies that looked at this phenomenon of school disengagement and delinquency over the course of middle adolescence, late adolescence and early adulthood. Researchers used a school disengagement warning index that summated standardized test scores, attendance, failure in one or more core subjects, suspensions and grade retentions. Results indicated that as a student accumulated more school disengagement risk indicators, the likelihood of dropping out of school increased dramatically. They found that dropout served as a significant full mediator on the effects of earlier school disengagement on serious violent crimes, official arrest/police contact, problem alcohol and drug use. Therefore, early school disengagement is associated with 
subsequent high school dropout, which is associated with serious problem behaviors in young adulthood.

More recently, research has been focusing on the association of physical and verbal school bullying and its relationship with long-term criminal behavior. As this topic continues to be integrated into criminological research, school bullying has been identified as an important risk factor that may suggest concern for social deviance (Bender \& Losel, 2011). Bullying is a power differential between the bully and target with the bully intended to do harm on the victim (Nansel, Overpeck, Haynie, Ruan \& Scheidt, 2003). A study by Bender \& Losel (2011) studied 87 young men recruited via the Erlangen-Nuremberg study to examine school bullying and victimization as predictors for offending and other anti-social behaviors in young adulthood. The study concluded that bullying at school was a strong predictor of self-reported violence, delinquency and other anti-social outcomes in young adulthood. Similar to these findings, Nansel et al (2003) conducted a study among 16,000 high schoolers in the United States investigating the relationship between bullying, possession of weapons and violent behaviors. They found a strong and consistent relationship between bullying and involvement in violent and aggressive behavior. Students who frequently bullied other peers had a higher chance for weapon possession and involvement in physical altercations. For boys who bullied peers atleast once a week, 52\% carried a weapon and $39 \%$ were involved in fighting.

Research has identified several risk factors that are linked to juvenile delinquency. These risk factors include: exposure to adverse life events and trauma, maternal smoking, maternal alcohol use, academic underachievement and school bullying. There is no 
evidence to support that a single risk factor will lead a young person to delinquency however the more risk factors that youth are exposed to the greater the likelihood that one will experience adverse outcomes such as delinquency (Barnert et al., 2015; Bradshaw, Schaeffer, Petras, \& Ialongo, 2010). Understanding how these factors influence one another can lead to the implementation of evidence based interventions that address modifiable risk factors such as academic underachievement and school bullying. Providing health education in the first trimester of pregnancy that addresses the impact of tobacco and alcohol abuse can also prevent potential adverse health outcomes in the developing fetus. Future research should further address intrinsic biological factors such as vagal tone and heart rate and its impact on emotional regulation. In addition to this, incorporating cognitive behavioral therapies that are geared towards assisting youth to learn how to cope and effectively address trauma can also be beneficial.

\section{Social Factors}

Antisocial behaviors can include non-adherence to social or family norms and may include: defiance, violence, stealing, property destruction or aggression (Hoeve et al., 2009). The theory of developmental psychopathology model of risky adolescent behavior suggests that children raised in high stress environments (poverty, discrimination, low neighborhood attachment and community disorganization who feel disconnected from teachers and peers and experience high levels of family conflict exhibit more behavioral problems that can result in juvenile justice involvement (Ellis et al., 2012). Although, there are some cases that fit outside of this theory, overall the model provides a framework for what is known about risky behaviors in adolescents. 
Parent-adolescent relationship quality illustrates aspects of parenting as well as aspects of parent involvement. Significant meaningful relationships with guardians, siblings and peers either serve to protect youth from engaging in risky behavior or lead to problems such as substance use and affiliation with deviant peers. Youth who feel close and connected to their guardian tend to value their opinion, support and guidance (Fosco, Stormshat, Dishion \& Winter, 2012; Ackard, Neumark-Sztainer, Story \& Perry, 2006). They also tend to spend more time with their families and have less opportunity to participate in delinquent behavior.

Family contexts characterized by structure, supervision, guidance and support facilitates a successful transition into positive relationships (Fosco et al., 2012). Parents who invest into their child's life, remain informed, supportive, and structure the adolescent's environment report observing fewer delinquent behaviors (Fosco et al., 2012; Hoeve et al., 2009). This process which is defined as parental monitoring, keeps parents aware of their adolescent's activities that allows the to respond appropriately to misbehavior. Parental monitoring decreases youth's experimentation with substances and involvement with delinquent behaviors (Fosco et al., 2012; Hoeve et al., 2009). Fosco et al (2012) investigated the impact of parental monitoring and youth relationships with their mother, father and siblings on problem behaviors. The results indicated that parental monitoring and family relationship each have important preventative roles in preventing anti-social behavior, substance use and engagement with defiant peers. Parental monitoring was also associated with a decrease in youth problem behavior. The study also revealed that sibling conflict contributes to the development of problem behavior. Youth who reported having more physical and verbal altercations with their siblings also 
reported increased problem behaviors by $8^{\text {th }}$ grade independent of the effects of parental monitoring and parent-youth relationships. This suggests relationships between youth; their parents and siblings individually play a critical role in pro-social development. Earlier research conducted by Dishion et al. (2003) found that parental monitoring was a mediating effect by which family interventions decreased adolescent substance use and delinquent behaviors.

Poor parent-adolescent relationship quality can result in disengagement between parents and their children that may further contribute to gravitation towards delinquency and deviant peers groups (Asscher et al., 2014). Barnert et al. (2015) performed a qualitative analysis in which they interviewed incarcerated youth to identify their perception of protective and risk factors in their lives. Participants identified their homes, schools and neighborhoods as chaotic, unstructured and unsafe, characterized by fighting and lack of family cohesiveness. Many youth felt neglected by their parents who were addicted to drugs, incarcerated or outside of the home working. The youth perceived that when their internal needs for love, attention, support, positive role models, discipline and control were not met in their home or school environments, they chose to spend more time in the streets engaging in risky behaviors. Participants identified love and attention as essential to helping youth stay out of trouble because affection from their parents motivated them to want to behave in order to please them. They also expressed a desire to have parents that provided them with a positive and future oriented perspective about life in addition to being supportive role models that guide their behaviors.

Research suggests that there is a relationship between involvement in a delinquent peer group and delinquent behavior (Monahan, Rhew, Hawkins \& Brown, 
2013; Fosco, Stormshat, Dishion \& Winter, 2012). Adolescence is a critical developmental time when the convergence of risky behaviors, substance use and affiliations with deviant peers may culminate thus developing into more pressing behavioral issues. As youth spend less time with families and more time with peers it is critical that they remain connected with their families to receive support and guidance through this vulnerable developmental stage (Fosco et al, 2012). The influence of peers on problem behavior may be more pronounced during adolescence likely because of the increased time spent with peers, the value in peer relationships and the susceptibility to peer influence (Monahan, Rhew, Hawkins \& Brown, 2013). A study conducted by Monahan et al (2013) condensed findings from twelve communities and seven states and found that peer problem behavior was a strong predictor of involvement in either delinquency or substance use. Steketee, Junger \& Junger-Tas (2013) analyzed data from an international self-reported delinquency study and concluded that family disruption and deviant behaviors of friends had more influence on females than males who were more influenced by a lack of self-control. Thus suggesting that females may be more vulnerable to certain peer group interactions and a propensity to want to "fit in and be accepted".

Social risk factors are circumstances related to the adolescent's immediate environment and include relationships with family and peers. Youth who identify having a meaningful relationship with their family and peers tends to avoid involvement with delinquent behaviors. Parents who provide support, guidance and structure set expectations regarding acceptable and unacceptable youth behavior. Households with less structure and parental monitoring fail to model they type of behavior that is expected of 
them. In addition to this, these youth often feel neglected and long for internal needs such as love, support, structure and discipline. As a result, they become exposed to more delinquent behaviors and connect with peer groups that engage in more risky behavior such as crime, violence and substance use. Research has established the value of positive pro-social relationships with parents and peers. However, future research must identify the factors that threaten pro-social relationships and ways to target these at-risk youth early, provide appropriate evidence based interventions and reduce the risk of long-term consequences.

\section{Community Factors}

The impact of neighborhood conditions on youth growth and development is a long-standing public health area of concern (Molnar et al, 2008). Juvenile violence is related to neighborhood characteristics such as high rates of families living in economic deprivation, high crime areas and violence rates, unemployment, female headed households, availability of drugs and large proportions of racial discrimination (Molnar et al., 2008; Wikström \& Loeber , 2000; Sampson \& Groves, 1989; Shaw \& McKay, 1942). The importance of neighborhood context in shaping involvement in crime has been acknowledged for decades. Some of the earliest work on neighborhood predictors of delinquency established the theory of "social disorganization," which linked high rates of concentrated poverty, ethnic heterogeneity, and resident turnover to higher rates of delinquency (Shaw and McKay, 1942 and Shaw and McKay, 1969). Shaw and McKay’s (1942) social disorganization theory, and expansions of it, are the dominant theories used to examine neighborhood influences on various criminological outcomes. Shaw and McKay discussed that three structural factors- low economic status, ethnic heterogeneity 
and residential mobility lead to the disruption of community social disorganization which in turn results in more crime, violence and delinquency. This theory states that the capacity of the community to control group level dynamics amongst peer groups is a key mechanism linking community characteristics with delinquency.

Researchers such as Sampson \& Groves (1989) have expanded this work and found communities characterized by sparse friendship networks; unsupervised teen groups and low organizational participation had disproportionately high rates of crime and delinquency. These factors along with poverty, family disruption and residential instability weaken social networks. Sampson (1997) proposed a social capital/collective efficacy model that links neighborhood context, family characteristics and individuallevel outcomes. He suggests that the extent to which adults are involved in stable social networks with relatives and other parents is a critical feature that affects parental socialization. Social networking is important since these group interactions provide opportunities for discourse related to discipline and parental monitoring, suggesting that family practice are mediated by the social organization of the community (Turner, Hartman \& Bishop, 2007). Neighborhoods plagued with high levels of poverty, family disruption and residential instability tend to weaken these social networks and the children typically are affected by the lack of socialization (Turner, Hartman \& Bishop, 2007). Collective efficacy is comprised of two components: social cohesion, the positive bonds formed between residents of the same neighborhood, and informal social control, the ability or willingness of neighborhood residents to monitor and/or intervene in activities occurring within the neighborhood (Tompsett, Amrhein \& Hassan, 2014; Sampson, 1997). Collective efficacy emphasizes that residents who trust one another and 
have similar values will guide behavior and act on each other's behalf to enforce societal standards. Collective efficacy is much more difficult to activate in areas marked by high rates of poverty and crime because the conditions of the environment discourage interaction amongst the residents (Tompsett, Amrhein \& Hassan, 2014; Losel \& Farrington, 2012; Sampson, 1997).

In addition to the relationships that residents share amongst one another, youth perception related to neighborhood conditions is also a vital factor. A study conducted by Unruh, Povenmire-Kirk \& Yamamoto (2009) used a qualitative design to examine the perceived risk and protective factors and their possible interactions within and across ecological domains from the perceptions of juvenile offenders. More than $50 \%$ of the youth identified access to drugs in their neighborhood as a barrier to successful community adjustment. Youth also identified a lack of healthy leisure activities such as safe parks, gym memberships and church attendance. Respondents reported when community resources were available and accessible they were less likely to engage in deviant behaviors. Building upon this work, a qualitative analysis by Barnert et al (2015) interviewed a group of incarcerated youth who identified their communities as needing more discipline and control to balance their hectic home environments. Participants described their ideal neighborhood as peaceful and quiet with parks and community members who were friendly to one another. Participants stated that their own neighborhoods promoted crime and was plagued with gangs, violence and shootings. The findings identified neighborhood poverty as the most important determinant for predicting juvenile offending because of the prevalence of negative influences in poor neighborhoods. Participants described an interrelationship between race and 
neighborhoods stating that it promoted pathways to jail. Neighborhoods with higher minority presence reported more police presence thus increasing the likelihood of arrests for minority youth.

Community risk factors are related to the impact of environmental factors on juvenile delinquency. Research has identified several features of a neighborhood that increases one's risk of involvement in delinquent behaviors. Poverty, violence, substance use, residential instability, and lack of supervision all weaken the community's cohesiveness and exposes youth to more delinquency and crime. In addition to this, residential instability, lack of social cohesion and poor informal social control discourages trust, interaction between neighbors and the motivation to improve the conditions of the environment. The perception of the neighborhood also illustrates whether residents identify their communities as having opportunities for growth or whether it's seen as a place that promotes crime, violence and delinquency.

\section{Protective Factors of Juvenile Delinquency}

Research on risk factors for delinquency has prompted discussion and identification of influences that may buffer the prevalence of delinquency. Protective factors offset the onset of delinquency through four main processes: reducing risk, reducing negative chain reactions, establishing self esteem and self efficacy and expanding new opportunities (Rutter, 1987). Protective factors can mediate or moderate the effect of exposure to risk factors thus resulting in a reduced incidence of problem behavior. Protective factors predict a low probability of delinquency in the presence of risk. These protective factors can be classified as individual, social and community factors. 


\section{Individual Factors}

Research focusing specifically on individual protective factors related to juvenile delinquency typically mentions the phenomenon of resilience. This concept describes the process of biological psychosocial adaptation to stressors and difficult circumstances in life (Rutter, 2012). The study of resilience was initiated from research on children who were at risk for mental health problems in an attempt to better understand etiologies of disorders (Masten 2007; Masten, Best \& Garmezy, 1990). Resilience refers to individual variations in response to risk. Garmezy was a pioneer in the area of resilience and studied children believed to be at risk for psychopathology due to environmental adversities and genetic vulnerabilities. He discovered that in spite of high- risk stressors or trauma, some children are still able to experience healthy growth and development. Ultimately, resilience research sought to better understand why some people succumb to stress and adversity and others are able to overcome the life (Rutter, 1987). Although resilience research has a broader focus, the phenomenon of juvenile delinquency can benefit from its theoretical implications. Understanding the role of resilience in juvenile offenders can help to better under the features of one's personality that protects their propensity to engage in juvenile delinquency.

High intelligence appears to have a direct protective function against problem behaviors (Vanderbilt-Adriance \& Shaw, 2008; Masten \& Coatsworth, 1998). The protective function of higher intelligence is related to better executive functioning, selfcontrol and social information processing. Executive functioning is an overarching construct encompassing a set of cognitive processing that allows for the achievement of goals and appropriate conduct (Portnoy, Chen \& Raine, 2013). Research also suggests 
that practical intelligence, social competence and realistic planning compared to abstract intellectual capacity protects against delinquent behaviors in the presence of risks. Children with higher intellectual capacity perform better at school, and academic success is associated with adoption of social norms and pro-social peer group development (Vanderbilt-Adriance \& Shaw, 2008; Masten \& Coatsworth, 1998). Early research conducted by Kandel et al., (1988) examined the protective effect of high IQ as tested by the Wechsler Adults Intelligence Scale in protecting high-risk men from criminal involvement. Results revealed that high-risk criminals who resisted criminal behavior had relatively high IQ scores. Similar to these findings, a longitudinal study conducted by Venderbilt-Adriance \& Shaw (2008) also identified child IQ as one factor associated with positive high social adjustment and low levels of delinquent behavior in adolescence. Portnoy, Chen \& Raine (2013) reviewed neuropsychological and psychophysiology factors that reduces the risk of antisocial behavior and identified high IQ as a direct protective effect.

Temperament relates to an individual's interaction with the environment and their ability to self-regulate his or her response. Research related to temperament identifies sociability, positive mood, low irritability and low impulsivity as protective factors against delinquent behaviors. A study conducted by Beaver, Delisi, Mears \& Stewart (2009) examined the relationship between self-control and the criminal justice system using data from the National Longitudinal Study of Adolescent Health. The results suggest that participants that displayed low self-control (which includes negative emotional control over one's temper) engaged in more deviant acts related to violent crime, property crime and substance use than participants who demonstrated more self- 
control. Early work by Moffitt et al (1996) studied a cohort of males from ages 3 to 18 and concluded that children who abstained from violence and antisocial behavior exhibited the easiest temperament during the preschool stage. Those who engaged in criminal activity were described as having more difficulty related to their temperament in childhood. This research along with similar findings led by Delisi \& Vaughn (2014) introduced a temperament-based theory of antisocial conduct with criminal justice behavior that identified effortful control and negative emotionality as two temper based constructs that are predictive of self-regulation deficits and behavioral problems from infancy to adolescence and across adulthood. Effortful control refers to one's ability to inhibit a dominant response and regulate attention, emotion and behavior to act accordingly with societal expectations (Delisi \& Vaughn 2014; Macdonald 2008). Negative emotionality is also known as negative affectivity which describes one's propensity for anger or irritability (Delisi \& Vaughn 2014). Negative emotionality is correlated with difficulties related to one's self-regulation. High levels of negative emotionality are associated with behavioral problems, difficulty disengaging from negative stimuli, disturbances in sleep, and mood dysregulation (Nakagawa \& Sukigara, 2012; Crockenberg, Leerkes, \& Barrig Jo, 2008).

Protective factors decrease the potentially harmful effect of a risk factor. Research has identified several individual protective factors that can reduce one's involvement in juvenile delinquency. Similar to risk factors, there isn't one protective factor that will prevent a youth's chance of involvement in juvenile delinquency. However, the collection of protective factors may decrease the adverse health consequences associated with juvenile delinquency. Individual protective factors related to resiliency, high 
intelligence and temperament have all been studied and identified as factors that reduce risk and promote pro-social development, self-efficacy and positive self-esteem. Future research must identify ways to target these at risk youth who lack the personality characteristics and create interventions which further promotes the development of resiliency and pro-social temperament. In addition to this, supporting youth to value education and the attainment of higher intelligence in order to reduce the risk of involvement in juvenile delinquency is vital.

\section{Social Factors}

A positive parent-child relationship and secure bonding is a direct protective factor in child development and has buffering protective effects in preventing a broad range of behavior problems in the presence of risk factors (Elliot et al., 2014; Losel \& Farrington, 2012). A positive relationship with at least one parent encourages social learning in a structured social environment and promotes positive growth and development (Elliot et al., 2014). A study conducted by Tompsett, Amrhein \& Hassan (2014) found adolescents who perceived adults in their home environment as having more pro-social values reported lower levels of delinquency. Research has also illustrated a positive correlation between parenting behavior and child delinquency (Elliot et al., 2014; Cicchetti, 2010). Parental monitoring strategies such as restrictive curfews, persistent discipline and strong involvement in the child's whereabouts particularly with peer groups has direct protective effects (Cicchetti, 2010). A study conducted by Herrenkohl et al (2005) found that parental disapproval of anti-social behavior predicted a low rate of violence and delinquency in both physically abused and non-abused children. An emotionally warm, supportive, accepting, supervising and structure-giving 
upbringing encourages positive growth and development (Tompsett, Amrhein \& Hassan, 2014; Losel \& Farrington, 2012).

In addition to parental involvement, school achievement, motivation and school bonding all have a direct protective effective against defiant behaviors. A study by Herrenkohl et al (2005) demonstrated that good school achievement had a buffering effect against violence and delinquency in a group of abused children. In children from high-risk backgrounds, sound school achievement and bonding to the school was more positively correlated to successful adjustment than high intelligence (Rutter \& Quinton, 1984). A later study by Herrenkohl, Lee \& Hawkins (2012) examined risk and protective factors associated with youth violence and delinquency. Direct protective factors at ages 10-12 and 13-14 included school attachment and other factors. For youth aged 15-18, one of the direct protective factors was high academic achievement. Although the direct causal relationship between school achievement and delinquent behaviors is unknown, school achievement opens the door to various noncriminal opportunities in one's life thus increasing one's chance to be a productive member of society (Herrenkohl, Lee \& Hawkins, 2012). Academic and professional motivation, desirable work behavior, and job stability has also demonstrated desistance from criminal activity in adulthood (Losel \& Farrington, 2012).

Deviant peers can both model and reinforce violent and delinquent behavior. Having a non-deviant good friend or being a member of a peer group who disapproves of antisocial behavior has a direct protective effect against youth violence and delinquency (Evans, Simons \& Simons, 2014; Herrenkohl, Tajima, Whitney \& Huang, 2005). Antisocial children are often rejected by others peers and as a consequence may join 
deviant peer groups (Losel \& Farrington, 2012). However, belonging to a peer group that encouraged pro-social behavior has a direct protective effect against engaging in delinquent behavior. Hoge, Andrews \& Leschied (1996) identified positive peer relations and effective use of leisure time as protective variables among a sample of youth offenders. A study by Evans, Simons \& Simons (2016) had similar findings and studied the trajectories to delinquent behavior and found respondents who reported having friends with greater delinquent behavior were more likely to be in groups that began offending early in life.

Research supports that having a positive relationship with one's parent/guardian can have a positive buffering effect against involvement in juvenile delinquency. Although, youth develop a sense of autonomy and spend more time with peers during adolescence, parents still hold a pivotal role in a young person's life. In addition to this, motivation to attend school and do well academically encourages youth to avoid involvement with delinquent peers. Belonging to a peer group that engages in pro-social behaviors and effectively uses their leisure time protects youth from the risk associated with juvenile delinquency. Providing after-school activities such as clubs and groups that encourage youth to learn new things, foster their interest and engage in social opportunities with their peers provides opportunities for positive social interaction. Teachers, school administration and community role models serve as examples as to how youth are expected to behave. Ensuring that youth have an opportunity to connect with a role model in the school setting can serve as a valuable resource and protective factor against involvement with deviant peers. 


\section{Community Factors}

Social disadvantage is partially reduced when people move to neighborhoods with improved housing and less violence, gang prevalence, crime and access to drugs.

Research suggests that it is not a single neighborhood characteristic but the accumulation of desirable features and subjective experiences that leads to protective effects against delinquency in a community (Elliot et al., 2014; Tompsett, Amrhein \& Hassan, 2014). Despite the many problems associated with disadvantaged communities, not all youth engage in or support criminal behaviors. Even in high poverty areas, residents can counteract the negative effects of disadvantage by engaging in collective efficacy, which involves making efforts to regulate the behavior of youth in a community (Sampson et al., 1997). Residents who interact and trust one another exercise informal social control to ensure that certain standards are enforced in the community (Tompsett et al, 2014).

Informal social control and social trust/cohesion are key neighborhood characteristics that promote nonviolence in various communities (Sampson et al., 1997).

Catalano \& Hawkins (1996) Social Development Model adds to the research related to social disorganization and states that the opportunity to bond with positive prosocial individuals in a community can be a protective factor against the development of delinquency. The findings from the Communities that Care prevention program revealed that when youth received more support and pro-social neighborhood values within adult figures and perceived that drugs and guns were less readily available they were also less likely to engage in delinquent behaviors (Arthur et al., 2007; Hawkins, Catalano \& Arthur, 2002). Neighborhoods plagued with high rates of violence and poverty offer youth fewer educational, social, and physical resources and fewer opportunities to learn 
new skills or interact with positive role models. These communities tend to be characterized by high rates of unemployment and single parent households that further reduces the time for the child and guardian to positively bond with one another. A study by Molnar et al (2008) also found that living in a neighborhood with a higher concentration of organizations or services serving youth and adults such as mentor resources was associated with lower levels of aggression and delinquent behaviors.

It is critical that neighborhoods are equipped with various community resources that can protect against the potential effects of living in a disordered neighborhood. Finding ways to address poverty, crime and violence within a community will impact one's subjective experience as a resident. Doing so may also encourage residents to interact and trust one another to ensure that certain standards are enforced in the community. Strengthening these relationships among residents and ensuring that they are connected with neighborhood resources can potentially reduce the risks associated with crime, violence and poverty. Future research related to neighborhood disorganization must identify interventions that target at-risk communities, addresses resident concerns and provides specific opportunities for growth via community outlets.

\section{History of Juvenile Delinquency}

The United Nations Convention on the Rights of the Child (UNCRC) was established in 1989 to recognize the rights of children worldwide (Muncie, 2009). The UNCRC proposed 40 specific rights advocating for the special protection of children, particularly those involved in the legal system that takes into account the special considerations associated with their developmental age (Muncie, 2009). In the early part

of the United States Progressive Era (1890-1920) the juvenile justice system was founded 
in order to address the unique needs of youth who had strayed from a moral path (Abrams, 2013; Lambie \& Randall, 2013). The justifications for incarcerating juveniles ranged from rehabilitation and punishment to providing a deterrent for future offending. By 1928, all US states had followed the trend established in Illinois and created their own separate juvenile justice system (Abrams, 2013). The state sets age limits that determine whether a person accused of a crime is treated as an adult or juvenile and most states consider juveniles to be under the age of 18 (Nisar, Ullah, Ali, \& Alam, 2015). Some states have reduced the limit to 16 and 17 , and juveniles charged with a serious crime such as murder or robbery can be transferred to criminal court and tried as an adult (Nisar et al., 2015). In addition to this, juvenile offenders cannot receive the death penalty, or receive a life sentence without parole except in the case of homicide (Lambie \& Russell, 2013; Fagan, 2008).

\section{Trends in Juvenile Diversion Programs}

In response to the increasing number of youth entering the juvenile justice system with mental health and substance use disorders and lack of comprehensive and adequate care many communities have created diversion programs as an alternative to incarceration. These programs provide assessments, thorough evaluations and access to comprehensive treatment. Diversion programs are based on the assumption that involvement in the juvenile justice system may do more harm than good and takes into account that adolescents have cognitive differences from adults, which has been support by adolescent brain development research (Abrams, 2013; Lambie \& Russell, 2013). Modern diversion practices trace their origin to the President's Commission on Law Enforcement and Administration of Justice (Gibbons \& Blake, 1976). The commission 
advocated the creation of formal channels to remove status offenders and first time offenders from traditional juvenile justice processing in order to minimize the effects of labeling and future offenses (Schwabe, Gearing, Mackenzie, Brewer \& Ibrahim, 2012).

Over the years, various diversion programs have focused on providing juvenile delinquents with interventions that are geared towards addressing their individual needs and reducing the chances for future offending. For any intervention to be effective, it must address all risk factors or criminogenic needs, and thus be tailored to the specific needs of the adolescent (Lambie \& Randell, 2013). The intensity and style of treatment must match the client's learning style and be capable of addressing target treatment goals. Interventions should be rehabilitative in nature, draw on evidence based behavioral techniques and focus specifically on the environment in which the adolescent lives (Lambie \& Russell, 2013; Henggeler \& Schoenwald, 2011). Family centered and community based approaches that address mental health needs are ideal because it assist the adolescent to develop the skill set and motivation to function adaptively and prosocially in society (Nisar et al., 2015; Lambie \& Russell, 2013; Henggeler \& Schoenwald, 2011). Interventions that effectively reduce the incidence of offending behaviors among youth are not only positive for the at-risk individual but also for their family and community (Henggeler \& Schoenwald, 2011).

\section{Successful Juvenile Justice Initiatives}

Over the past decade, the juvenile justice system has witnessed a shift from a belief that "nothing works" for youth in the juvenile justice system to a recognition that evidence based community programs have demonstrated positive short and long term outcomes (Phillippi, Cocozza \& Deprato, 2013; Henggeler \& Schoenwald, 2011; Lipton, 
Martinson \& Wilks, 1975). The landmark Juvenile Justice and Delinquency Prevention Act of 1974 promoted both deinstitutionalization and diversion programs particularly for lower level and first time offenders (Abrams, 2013). There is evidence that suggest that the formal processing in the juvenile court system may place adolescents on more of a criminal path than if they were released or diverted (Petitclerc, Gatti, Vitaro \& Tremblay, 2013). These programs are designed for a variety of reasons including reducing future involvement with the court system, decreasing court expenses and reducing the stigma associated with having a criminal record (Seroczynski, Evans, Jobst, Horvath \& Carozza, 2015). Several cities have demonstrated an increase in effective programs designed to improve functioning, decrease association with deviant peers and incorporate behavioral interventions within the adolescent's natural environment (Henggeler \& Schoenwald, 2011). The Blueprints for Healthy Youth Development reviewed over 600 delinquency, drug and violence prevention and intervention programs and identified Multisystemic Therapy (MST), Functional Family Therapy (FFT) and Multidimensional Treatment Fostercare (MTFC) as effective programs that do not involve incarceration (Lambie \& Randell, 2013; Schwalbe et al., 2012; Henggeler \& Schoenwald, 2011; Elliott, 1998). The effectiveness of a diversion program rests on its ability to assess the problems that an adolescent is experiencing and coordinate services that adequately addresses the adolescents needs (Abrams, 2013).

Greenwood (2008) concluded that only about $5 \%$ of juvenile offenders have the opportunity to benefit from programs with proven effectiveness. Effective programs such as Functional Family Therapy (FFT), Multisystematic Therapy (MST), and Multidimensional Treatment Fostercare (MTFC) each use behavioral and cognitive 
behavioral intervention techniques to address and improve the functioning of justice involved youth and their family members. Functional family therapy (FFT) is a family and community based program identified as one of the first evidence based treatments in the juvenile justice field (Henggeler \& Schoenwald, 2011). FFT views the presenting conflict as a factor of dysfunctional family relations and engages and motivates family members to establish new patterns of behavior to replace deviant behaviors (Henggeler \& Schoenwald, 2011). Multisystemic therapy (MST) is also a community and family based treatment that focuses on youth with co-occurring substance and mental health issues. MST functions similarly with the Bronfenbrenner Theory of Social Ecology (1979) and acknowledges that youth are nested within multiple systems that have direct and indirect influences on their behavior. Interventions are comprehensive and designed to address factors at the individual, family, peer, school and community level. Multidimensional treatment foster care provides community-based foster care as an alternative to state detention. This treatment recognizes the critical role of parental supervision and parental monitoring to support youth to engage in pro-social activities and disengaging from deviant peers in order to achieve positive academic achievement (Henggeler \& Schoenwald, 2011).

Several validated programs have demonstrated effectiveness to reducing various concerning outcomes associated with juvenile delinquency. One of the most well-known and respected programs for child delinquents is the Stop Now and Plan (SNAP) (Burke \& Loeber, 2015; Farrington, 2012). SNAP was developed in the 1970's and formalized in 1985 in an attempt to reduce antisocial behavior among children 6-11 years old (Burke \& Loeber, 2015). The program incorporated the Toronto police department, fire 
department, child welfare agencies and schools to establish a single-entry access through the Child Development Institute to conduct assessments and formulate treatment options (Farrington, 2012). SNAP helps children and parents to regulate angry feelings by getting them to stop, think and plan prior to acting out aggressively and impulsively (Farrington, 2012). A study by Burke \& Loeber (2015) conducted a randomized controlled treatment effectiveness study of SNAP compared to standard behavioral health services. The treatment program was delivered to youth with aggressive, rule breaking or antisocial behaviors and measured outcomes at 3,9, and 15 months from baseline. Youth in the SNAP program demonstrated less aggression, conduct problems and externalizing behaviors than the standard behavioral health services group. The program was also more effective in reducing the behavioral problems experienced by youth with more severe problems. Significant differences were also found for internalizing behaviors such as aggressive behavior, ODD (oppositional defiant disorder), ADHD (attention deficit hyperactivity disorder), depression and anxiety symptoms for youth involved in the SNAP program. The findings suggest that the SNAP program was more effective at reducing high-risk behaviors in comparison to other behavioral health interventions.

Some juvenile justice diversion programs have implemented unique and innovative alternatives that do not involve the court system. Serocynski et al. (2015) evaluated the impact of Reading for Life (RFL), a program that allows low-status juveniles to study works of literature in small reading groups led by mentors. The program strives to foster moral development in at-risk adolescents through moral discussion and engaging, educating and empowering its participants. Results demonstrated a statistically significant reduction in the probability of reoffending by 12.1 
percent. RFL was most effective at reducing more serious offenses by groups at higher risk to recidivate and reduced prosecuted offenses by 50.9 percent. Similar to these findings, a longitudinal study conducted by Matthews, Krivelyova, Stephens \& Bilchik, (2011) found that probability of juvenile justice contact decreased from 0.64 to 0.36 for youth involved in the system of care verses the services as usual environment. System of care offers a wide array of services that are accessible, community based, culturally competent and individualized. The findings are suggests that services should be offered in the least restrictive environment. The final study conducted by Wilson \& Hoge (2013) evaluated the Ottawa Community Youth Diversion Program (OCYDP) that targets at risk youth and individualizes the program based on risk and need. The recidivism rate of 170 youth referred to the OCYDP were compared to that of 208 matched youth sentenced to a period of probation. Youth referred to diversion had significantly lower re-offense rates than those referred to probation even when controlling for risk level, age, gender, and nature of the index offense. There were no significant difference in recidivism rates after 6 months or 12 months, however there were significant differences in reconvictions after 18 months and through the entirety of the follow up period. Petitclrc, Gatti, Vitaro \& Tremblay (2012) reviewed longitudinal data from a study that followed over one thousand boys from ages 6-25 years of age. The study showed that male adolescents processed in juvenile court had three times the odds of being convicted of an adult crime by age 25 and committed twice as many violent and non-violent adult offenses compared to matched peers who were arrested but not involved in the court system. 


\section{Outcomes of the Ohio Behavioral Health Juvenile Justice}

Ohio's Behavioral Health Juvenile Justice (BHJJ) initiative was created to address adolescents with behavioral health issues who often have inadequate and limited access to care to address their needs. The BHJJ initiative is funded by a partnership between the Ohio Department of Youth Services (ODYS) and the Ohio Department of Mental Health and Addiction Services (ODMHAS). The BHJJ program diverts youth from local and state detention centers into more comprehensive, intensive, community based mental health treatment. The BHJJ enrolls youth 10-18 year of age who meet the following criteria: DSM IV Axis 1 Diagnosis, substantial mental status impairment, co-occurring substance use/abuse problems, criminal or violent behavior and a history of multi-system involvement. The goal of the BHJJ initiative was to transform and expand the Ohio justice systems ability to identify, assess, evaluate and treat youth with juvenile justice involvement in more effective, evidence-based treatment options.

According to the Ohio Youth Assessment System (OYAS), a criminogenic risk assessment tool, $71 \%$ of the youth enrolled in the BHJJ were identified as moderate or high risk and $35 \%$ of youth had a felony charge 12 months prior to program enrollment. In addition to this, $70 \%$ of the youth were suspended or expelled in the year prior to their enrollment and $94 \%$ of the youth were attending school at termination. At intake, 36.3\% of participants had A's, B's and C's while $34.7 \%$ were earning either a D or F. At termination from the BHJJ program, $49.3 \%$ were receiving A's, B's and C's and 19.1\% were receiving either D's or F's.

An evaluation of the BHJJ program conducted by Kretschmar, Butcher, Flannery and Singer (2014) found that all of the youth involved in the BHJJ program had a mental 
health diagnosis and $40 \%$ were diagnosed with a co-occurring substance use disorder. In addition to this, $15 \%$ of the youth admitted to attempting suicide in the past. The study found that non-white youth, youth with higher scores on the substance use index and youth with co-occurring mental health and substance use disorders were less likely to complete treatment successfully. Caregivers reported that the youth had been exposed to domestic violence and suffered from physical and sexual abuse in the home. Overall, youth involved in the program exhibited statistically significant improvements in trauma symptoms, psychological functioning and problem severity from enrollment to termination. The study also found that $92 \%$ of caregivers were satisfied with the services they received while their children were engaged in the BHJJ program.

\section{Mental Health and Juvenile Delinquency}

Adolescent offenders enter the juvenile justice system with multiple needs that include psychiatric treatment, medication management, intensive case management and outpatient services (Colwell, Villarreal \& Espinosa, 2012). Between 70\% and 95\% of detained youth offenders suffer from mental health problems such as depression, anxiety, ADHD, suicide attempts and ideation, as well as drug and alcohol abuse. (Lambie \& Randall, 2013; Colwell et al, 2012). There is considerable evidence suggesting that the prevalence of emotional and behavioral problems among adolescent offenders may be four to six times greater than the general adolescent population (Lambie \& Randall, 2013; Schubert, Mulvey, \& Glasheen, 2011). For many youth with serious emotional or behavioral problems, the justice system may be their first point of contact for mental health support and often is their only access to care (Chapman, Desai, \& Falzer, 2006). The juvenile justice system was not originally designed to address the challenges 
associated with mental health disorders and juveniles entered a system that was ill equipped to address their psychosocial concerns and complex medical diagnoses (Colwell et al, 2012; Callahan, Cocozza, Steadman \& Tillman, 2012; Cocozza \& Skowyra, 2000). Over the years, the justice system has made great efforts to add additional services and assessment protocols in order to better address the concerns associated with mental health and substance use amongst juvenile offenders (Liebenberg \& Unger, 2014; Hoeve, McReynolds, \& Wasserman, 2014). While research consistently indicates higher levels of mental health needs among youth offenders, evidence suggests that youth offenders do not receive appropriate referrals and interventions to address their mental health challenges (Whitted, Delavega \& Lennon-Dearing, 2013; Wasserman et al., 2008). Youth have also self-reported lower levels of interactions with mental health services and educational support (Liebenberg \& Unger, 2014). This service gap is particularly troubling since youth are at a greater risk of reoffending when they have multiple mental health diagnoses (Hoeve et al., 2014).

\section{Depression among Juvenile Offenders}

Youth involved in the juvenile justice system present with mental health concerns that can include: anxiety, depression, ADHD, ODD, mood disorder and substance use. Among these psychiatric conditions, depression is particularly concerning seeing that unrecognized and untreated depression impacts the criminal course and increases recidivism rates (Schubert, Mulvey \& Glasheen, 2011; McCarty, Stoep, Kuo, \& McCauley, 2006). Depression is often "masked" particularly among youth who present with aggression, violence and disruptive behaviors (Liebenberg \& Unger, 2014; Ryan \& Redding, 2004). Depression may also interfere with a youth's engagement in a treatment 
program and adherence to their medication regimen (McCormick, Peterson-Badali \& Skilling, 2015). A study conducted by Asarnow et al (2014) found that depressive disorder was significantly associated with more high-risk indicators such as tobacco use, drug and alcohol misuse and risky sexual behaviors. Depression treatment was also complicated by high-risk behaviors and reduced substance use was associated with improved depressive symptoms.

\section{Suicidal Ideation among Juvenile Offenders}

Depression is the leading cause of disability worldwide but it is also associated with suicide, the third leading cause of death in adolescents and young adults (McCormick, Peterson-Badali \& Skilling, 2015; Asarnaw et al., 2014). Adolescents in juvenile detention often have higher rates of risk factors for suicide compared to the general population of youth such as mental illness, trauma and adverse life events (Bhatta et al., 2014). Suicide is the leading cause of death among adolescents in juvenile detention centers with rates 3 to 18 times higher than the general population of adolescents (Bhatta et al., 2014; Fazel, Benning \& Danesh, 2003). The prevalence of suicidal ideation ranges from $22-58 \%$ and lifetime suicide attempts ranging from 11-33\% (Bhatta et al., 2014). A study conducted by Bhatta et al (2014) assessed the influence of multiple adverse life experiences (sexual abuse, homelessness, running away and substance abuse in the family) on suicidal ideation and suicidal attempts among youth at a detention center. The findings revealed that over $19 \%$ of the participants reported having suicidal ideation and $11.9 \%$ reported attempted suicide. The key findings revealed that those with a history of sexual abuse, homelessness, running away and substance use was 5.7 to 7.8 times more likely to report suicidal ideation and attempted suicide. 


\section{Mental Illness affect on Functioning}

The majority of youth in the juvenile justice system have psychiatric, social and academic difficulties that can impair their day-to-day functioning (Teplin et al., 2005). Diagnosis of mental illness and substance use disorders can lead to disorganization of thought and impaired, impulsive decision making with a propensity to not fully understand the implications of one's decisions (Colwell, Villareal \& Espinosa, 2012). Youth with depression often experience feelings of hopelessness and worthlessness and show impaired social functioning (Schubert, Mulvey, \& Glasheen, 2011). Functional impairment refers to a youth's day-to-day social, psychiatric, and academic difficulties. Functional impairments may also make it challenging for justice involved youth to follow basic rules of the court, requirements of probation and participate in their own defense (Colwell, Villareal \& Espinosa, 2012; Teplin et al., 2005; Kazdin, 2003). Consequently, these youth are at a higher risk to not successfully complete their court requirements unless systems are put into place that make the appropriate accommodations, such as those considerations found in a diversion program. A well-known longitudinal study conducted by Glueck \& Glueck (1968) analyzed 500 incarcerated juvenile boys (7-11 years) and compared them to 500 non-delinquent males residing in the Boston area. The researchers found that by adulthood, few delinquent males had graduated from high school and they were also less likely to be employed. Abrams et al (2013) was the first large scale longitudinal study that examined youth functional impairment at 3 months and three years after they were initially assessed. More than one-fifth of the sample had marked global functional impairment and more than one-third were severely impaired in the school/work domain. Three years after detention, approximately one of every five 
youth had impaired functioning and needed appropriate intervention and care after release. Early research analyzing the impact that mental illness and depression has on function in juvenile justice involved youth has led to the development of interventions that improve outcomes. A study conducted by Colwell, Villareal \& Espinosa (2012) used specialized juvenile probation officers to divert youth from jail and linked them to community services. The officers participated in courses related to motivational interviewing, family engagement, crisis intervention, and behavioral health management. The researchers discovered that youth who received specialized services had improvements in functioning on the Global Assessments of Functioning and Ohio Scales. They also had improved school attendance and fewer disciplinary actions. Pre and postscores on the Ohio Scales indicated that perceived problem severity not only decreased among the juveniles but also for parents and probation officers. The youth had an improvement in perceived functionality with the youth, parents and juvenile probation officer. Parents and juveniles who received specialized supervision were satisfied with the services provided as well as their intensive case management.

\section{Benefits of Social Support on Mental Illness}

Two important predictors of depressive symptoms among youth are stressful life events and lack of parental support (McCarty, Stoep, Kuo \& McCauley, 2006). There is evidence that low levels of parental support and low family cohesion are associated with depressive symptoms (Patten et al., 1997). Social support has a negative association with emotional distress and a positive association with pro-social development (McCarty et al., 2006). A study conducted by McCarty et al. (2006) sought to examine the relationship between caregiver support, stress and youth self reported depressive symptoms. 
Perceived caregiver support was associated with youth self report of depressive

symptoms regardless of stress levels. Participants who identified having less supportive parents reported more depressive symptoms. The findings are consistent with evidence suggesting that social support has a positive impact on depression even among youth exposed to multiple risk factors.

In addition to parental support, peer groups form a critical context for healthy growth and development. Peer relationships influence various youth behaviors such as aggression, delinquency, depression and substance use (Asscher et al., 2014; Evans, Simons \& Simons, 2014; Assink et al., 2015). A study conducted by Reynolds \& Crea (2015) explored the multiple factors that account for peer influence related to adolescent delinquency and depression. The results revealed that both peer delinquency and depression are predictors of youth delinquency. For delinquency, school connection and attachment to parents intensified peer effects while self-regulation decreased peer effects. This suggests that youth who are able to regulate and control their emotions and behaviors were less likely to be influenced by deviant peers. Therefore, for youth who have depressive symptoms and have less self-regulatory skills they are particularly vulnerable to peer influence and at increased risk for engaging in deviant behavior (Reynolds \& Crea, 2015).

\section{Adolescent Substance Use (Trends and Relationship to Juvenile Delinquency)}

Adolescent substance use is a widespread public health concern that threatens the safety, health and functioning of our youth. It is critical to understand the developmental processes that contribute to adolescents' anti-social behaviors and substance use because of the implications that these problems have on their health and the burden to society 
(Schulenberg, Patrick, Maslowsky \& Maggs, 2014; Fosco, Stormshak, Dishion \& Winter, 2012). The National Center on Addiction and Substance Abuse (2009) reports that the U.S government spends $\$ 467.7$ billion each year on the consequences of substance use and less that two percent of this funding is allocated towards prevention and treatment. Adolescents are at particular risk because the prefrontal cortex and reward pathways are still developing thus explaining the increased propensity to take risks such as smoking, drinking or using drugs (Feinstein, Richter \& Foster, 2012). The earlier an individual engages in substance use, the greater the likelihood of developing a substance use disorder (Feinstein et al., 2012). The National Survey on Drug Use and Health indicates that 9 of 10 people who meet the criteria for a substance use disorder began using tobacco, alcohol or other drugs before the age of 18 (U.S Department of Health and Human Services, Substance Abuse and Mental Health Services Administration, 2014). Those who use tobacco, alcohol or other drugs before age 15 are 6.5 times more likely to develop a substance use disorder than those who delayed use until age 21 or older (U.S Department of Health and Human Services, Substance Abuse and Mental Health Services Administration, 2014).

\section{Substance Use Relationship to Juvenile Delinquency}

Substance use impairs decision-making ability, lowers inhibitions, increases impulsivity and reduces perceptions of personal risk (Schubert, Mulvey \& Glasheen, 2011). Adolescent substance use also impacts academic performance, career achievement and social functioning (Feinstein, Richter \& Foster, 2012). In a study conducted by Hoeve, McReynold \& Wasserman (2009) youth with substance use disorders not only committed more re-offenses but also more severe re-offenses compared to youth who did 
not engage in substance use. In addition to this, youth who had a substance use disorder were a third more likely to commit more property crimes (burglary, larceny, theft, arson, vandalism) compared to counterparts without substance use disorder. Similar findings were found in a study conducted by Schubert et al (2011) that concluded that participants with substance use disorders had higher rates of recidivism and more self-reported antisocial behavior and lower functioning. More than 63 percent of detainees with any substance use disorder, either alone or with a comorbid mental health disorder, engaged in five or more risky health behaviors (Teplin et al., 2005). Teplin et al (2005) found that among youth with comorbid mental and substance use disorders, $96 \%$ had been sexually active, $62 \%$ had multiple partners within the past three months, and $59 \%$ had unprotected vaginal sex in the past month. The high prevalence of substance use amongst juveniles is particularly troubling because youth who engage in these risky behaviors have an increased likelihood of adverse outcomes such as: poor physical health, lower life expectancy, poor psychosocial development and difficulty with transitions into adulthood (Monahan, Rhew, Hawkins \& Brown, 2013). Delisi et al (2013) examined twelve forms of juvenile delinquency (murder, rape, robbery, aggravated assault, burglary, larceny, auto theft, arson, weapons, sexual offense, drug sales and drug use to explore the association with total arrests. The only offense that was significantly associated with all criminal career outcomes was juvenile drug use. Delinquency has also been identified as a gateway to substance use and most youth who engage in deviant behaviors start with delinquency that escalated to co-occurring substance use (Monahan et al., 2013). 
Age of Onset of Substance Use

One consistent predictor of early-adult substance abuse is early initiation of alcohol and drugs. Research suggests that those who use a substance early in adolescence are at a greater risk to have more problems with substances as young adults (Stone, Becker, Huber \& Catalano, 2012; King \& Chassin, 2007; Gil, Wagner \& Tubman, 2004). Gil et al (2004) found that regular substance users who began using substances in early adolescence were 1.5 times more likely to experience alcohol abuse and 2 times more likely to abuse marijuana and be classified as having a substance use disorder in adulthood. Later research by King \& Chassin (2007) resulted in similar findings and concluded that youth who began substance use prior to the age of 13 were 3.16 times more likely to develop drug dependence in adolescence. With alcohol use, age of initiation and chronicity of use have both been associated with negative outcomes such as aggressive behaviors, delinquency and suicidal ideation (Duncan et al., 1997). A study by Guttmannova et al. (2011) examined the association between age at alcohol use onset and adult alcohol misuse and dependency during the critical developmental period of ages 1114. Results suggest that onset of alcohol use before age 11 when compared with initiation between ages 11-14 related to increased chronicity of adult alcohol dependence. Those who initiated alcohol use before age 11 had a 1.62 greater risk of alcohol dependence than those who delayed initiation until they were 15-17 years old and 3.67 times greater risk than those who did not start drinking until they were 18-20 years old. The findings also suggest that frequent marijuana use in adolescence predicted an increased likelihood of lifetime alcohol misuse over the course of young adulthood. Research has suggested that early onset of drug use produces more risk for drug dependence than many familial 
and behavioral risk factors such as familial alcoholism, parental support and family conflict (King \& Chassin, 2007; King \& Chassin, 2004).

\section{Influence of Neighborhood Conditions on Substance Use}

Neighborhoods characterized by areas of economic and social deprivation experience more delinquency, crime, violence and substance use than more affluent neighborhoods (Fagan, Wright, \& Pinchevsky, 2015; Sampson \& Groves, 1989; Shaw \& McKay, 1942). This may in part be due to availability of drugs or the social norms illustrated in more disorganized neighborhoods that give the perception that substance use is accepted. Whether it is through social networks or drug activity, youth living in disorganized neighborhoods reside in environments that both promote and normalize substance use (Handley, Rogosch, Guild \& Cicchetti, 2015; Reboussin, Green, Milam, Furr-Holden \& Ialongo, 2014). Research investigating neighborhood influences rely on both objective (U.S census data) and subjective (youth and/or parent report of neighborhood conditions) to measure the level of disadvantage in a community. In a study conducted by Fagan \& Wright (2012) they examined whether neighborhood characteristics influenced youth's attitudes about tobacco, alcohol and marijuana use and whether neighborhood norms about deviance moderated the impact of youth's perception on their own drug use. Results concluded that youth living in communities that were generally favorable to deviance and drug use believed that substance use was less harmful to their health than youth living in communities that were less tolerant to deviance and drug use. Research that has utilized subjective resident report of neighborhood conditions related to disorder, cohesion and safety have demonstrated a correlation between neighborhood disorganization and adolescent substance use. A study 
conducted by Tucker, Pollard, De la Haye, Kennedy \& Green (2013) examined whether census-based indicators of neighborhood disorganization as well as adolescent subjective report related to their neighborhood predicted their initiation of substance use over a oneyear period. Results revealed that residing in an area with higher unemployment increased an adolescent's risk for initiating marijuana use. Adolescent's who perceive their neighborhoods as offering less opportunity for their future become hopeless and may choose to experiment with substances (Tucker et al., 2013). Substance use may also provide youth with a means of coping with the chronic stress of living in disordered and violent neighborhoods that are poverty stricken in the absence of having protective neighborhood social support. Reboussin et al. (2014) found that disordered neighborhoods, exposure to drug activity, and exposure to violence in the community were associated with higher levels of depressed mood as well as an increased likelihood of transitioning from no use to infrequent and frequent use of substances. Higher levels of depression partially mediated the relationship between disordered neighborhoods and exposure to community violence and completely mediated the relationship between drug activity and sales and initiation of more frequent use.

Neighborhood structure refers to the characteristics of the community while neighborhood social processes refer to a community's social cohesion (Trucco et al., 2014). Both of these factors impact the composition and character of a community. A lack of structured community organizations weaken bonds to pro-social activities that adolescent's can partake in during their unstructured time (Trucco et al., 2014). Neighborhood disorganization may also compromise parental monitoring where there is a lack of consensus regarding social norms and acceptable behavior among neighborhood 
residents. A study conducted by Cleveland, Feinberg, Bontempo \& Greenberg (2008)

compared the relative influence of risks and protective factors across several domains on adolescent substance use and found that although peer influence was a strong risk factor, community was the strongest protective factor against substance use for youth.

Neighborhood attachment, community pro-social activities and laws that are enforced that set clear boundaries and expectations for youth can decrease one's risk of substance use even in the presence of more neighborhood disorder. Lo, Webar \& Cheng (2015) sought to delineate whether social organizational factors mediated and/or moderated structural disadvantages on student substance use. Researchers concluded that community encouragement and rewards for desirable behavior from adolescents reduced adolescent substance use even when the youth attended a school in a disorganized neighborhood. Thus suggesting that even in the midst of a structurally disadvantaged community, investing into the social capital relative to the neighborhood such as community resources and public spaces can reduce adolescence deviance and problematic behavior.

\section{Substance Use Trends (Alcohol, Tobacco and Marijuana)}

Monitoring the Future (MTF) is a longitudinal epidemiological study started in 1975 at the University of Michigan Institute of Social Research and funded by the National Institute on Drug Use (NIDA) (Johnston, O’Malley, Miech, Bachman \& Schulenberg, 2016). The purpose of this study is to explore changes in attitudes, behavior and lifestyles orientations of American youth. Since the leading cause of death among adolescents (15-19 years) is unintentional injury early identification of substance use and intervention decreases the risk for adverse health outcomes in adulthood. While the developing brain may be more resilient to neurotoxic effects, exposure to substances 
during the critical neurological development can disrupt the natural course of brain development (Squeglia et al., 2014). Substance use during adolescence has been associated with alterations in brain structure, function and neurocognition (Moss, Chen \& Yi, 2014; Trucco, Colder, Wieczorek, Lengua, \& Hawk Jr, 2014). Teenage substance use is also associated with various high-risk behaviors that increase their risk for injury or involvement with the juvenile justice system. The MTF study analyses American adolescents and college student's substance use patterns and beliefs. The study also examines perceived availability of drugs and social norms among peers and social groups. Lastly, the study explores the subjects attitudes related to occupational and educational goals, parental influences, self-esteem, religion, attitudes related to gender roles, concerns about the environment, media, victimization and delinquency. Follow up questionnaires administered post graduation covers marriage, family, college attendance, military service and unemployment.

\section{Alcohol Use among Adolescents (Trends \& Effect)}

Alcohol is a commonly abused central nervous system depressant that can impair brain function and motor skills (Moss, Chen \& Yi, 2014; Graydanus \& Patel, 2005). According to the MTF study, the proportion of $8^{\text {th }}, 10^{\text {th }}$ and $12^{\text {th }}$ graders who reported drinking alcohol within the month prior to the study was $10 \%, 22 \%$ and $35 \%$ (Johnston, O’Malley, Miech, Bachman \& Schulenberg, 2016). Alcohol use changes throughout adolescence and differs not only by age but also by gender, race/ethnicity and grade level (Patrick \& Schulenberg, 2014). Although the perceived availability of alcohol use has declined since 1996, there is still concern with adolescents regarding patterns of alcohol consumption and binge drinking. Binge drinking refers to consuming five or more drinks 
or one on more occasions in the past two weeks (Johnston et al., 2016). Research suggests that there are sensitive or vulnerable times during early adolescent brain development when the effects of alcohol onset are particularly detrimental (Guttmannova et al., 2010). Early alcohol use has been associated with several neuropsychological deficits related to memory, executive function, visuospatial skills and sustained attention (Trucco, Colder, Wieczorek, Lengua, \& Hawk, 2014). A study conducted by Squeglia et al (2014) used high-resolution magnetic resonance imaging (MRI) to evaluate cortical volume changes in youth who reported heavy alcohol use versus those who sustained from using alcohol. The study found that heavy drinkers exhibited more brain volume reduction compared to non-drinkers. The regions showing alcohol related volume reductions were important areas for sensory integration, motor control, feedback processing, language comprehension and visual object reductions.

\section{Tobacco Use Among Adolescents (Trends and Prevalence)}

Cigarette smoking is usually initiated in adolescence and is the leading cause of preventable disease and mortality in the United States (Johnston, O’Malley, Miech, Bachman \& Schulenberg, 2016). Nicotine is a chemical compound found in tobacco that is not only highly addictive but also adversely effects lung function and can lead to chronic cough, impaired lung growth, wheezing and shortness of breath (http://tobaccofreekids.org). Long-term effects of tobacco use include chronic lung disease, cancer, gum disease, tooth decay, heart disease and stroke (http://tobaccofreekids.org). According to the U.S Department of Health and Human Services (2014) if current smoking patterns persist, well over 5 million of todays youth in the United States will die prematurely from tobacco related illnesses. Historically, 
reported tobacco use continues to increase in American youth through 8 to $12^{\text {th }}$ grade. In the 1990 's, smoking began to rise among high school students and peaked in 1996 for $8^{\text {th }}$ and $10^{\text {th }}$ graders and 1997 for $12^{\text {th }}$ graders. The last MTF report found $13.3 \%$ of $8^{\text {th }}$ graders, $19.9 \%$ of $10^{\text {th }}$ graders and $31.3 \%$ of $12^{\text {th }}$ graders reported any time use of cigarettes.

Electronic Cigarettes (E-Cigarettes) is a relatively new nicotine delivery system that has become an increasingly popular emerging health hazard. E-Cigarettes are battery powered and have a heating element that produce aerosol and vapor that users inhale. The MTF study included new questions about adolescents using vaporizers such as ecigarettes in 2014. E-cigarette use continues to gain popular, as less youth perceive less risk associated with e-cigarette use. According to the MTF study, e-cigarette use had the lowest perceived risk associated with regular use compared to illicit drugs and alcohol. In 2015, e-cigarettes continued to have higher use among youth than traditional cigarettes. The 30 -day prevalence among $8^{\text {th }}, 10^{\text {th }}$ and $12^{\text {th }}$ graders was $9.5 \%, 14 \%$ and $16.2 \%$. E-cigarettes use among youth offsets a long-term decline in the use of tobacco cigarettes that is at a historic low among U.S adolescents.

\section{Marijuana Use Among Adolescents (Trends and Incidence)}

Marijuana continues to be the most popular illicit drug used among adolescents (Johnston, O’Malley, Miech, Bachman \& Schulenberg, 2016). The health and social consequences from marijuana use are a serious concern for developing adolescents and has shown to be associated with increased rates of addiction, cognitive impairments, poor educational outcomes, risky sexual behaviors and altered brain development (Reboussin, Green, Milam, Furr-Holden \& Ialongo, 2014; Rubino, Zamberletti \& Parolaro, 2012). 
Regular marijuana use effects fine neural refinement, which impacts learning, memory, attention and spatial working memory (Rubino, Zamberletti \& Parolaro, 2012; Medina et al., 2007). Research suggests that heavy marijuana use in adolescents may also cause adverse psychosocial consequences such as cognitive abnormalities (memory loss), psychotic symptoms (paranoia) and increased risk for mental illness (depression and anxiety) (Di Forti et al., 2007). Adolescents who regularly used marijuana may be at heightened risk of developing psychotic symptoms (Bechtold et al, 2016; Mandelbaum \& de la Monte, 2017). A recent study by Bechtold et al (2016) found that for every year adolescent boys engaged in regular marijuana use their expected level of psychotic symptoms increased by $21 \%$. Since schizophrenia and psychosis share similarities and both present in late adolescence there is growing concern that adolescent cannabis use may cause or trigger early psychosis and schizophrenia presentation (Mandelbaum \& de la Monte, 2017). A study conducted by Wilcox \& Anthony (2004) found that marijuana use in girls under the age of 15 years significantly raised their suicidal risk or attempt in the following 15 year follow up. A later study conducted by Squglia, Jacobus \& Tapert (2009) found that adolescents who regularly smoked marijuana performed poorer on performance tests of learning, cognitive flexibility, visual scanning, error commission, and working memory. Annual marijuana use among $8^{\text {th }}$ graders increased from 2007 to 2010 and decreased from 2010-2012 and then leveled. The increase in use is in part related to availability in addition to a low perceived risk associated with marijuana use. Since the MTF study began in 1975 , between $80-90 \%$ of $12^{\text {th }}$ graders stated that marijuana was easily accessible. In 2015 , daily use increased with one in seventeen $12^{\text {th }}$ graders reporting daily marijuana use. 


\section{Substance Use and Depression}

There is evidence supporting the notion that depression has bidirectional relationships with several unhealthy health behaviors such as alcohol, tobacco and marijuana use (Katon et al., 2010; Brook, Brook, Zhang, Cohen \& Whiteman, 2002; Kandel, Raveis \& Davies, 1991). Depression is a mental health disorder that increases one's risk of poor academic performance, teen pregnancy, violent or aggressive behavior, suicide, substance use and involvement with the juvenile justice system (McCormick, Peterson-Badali \& Skilling, 2015; Schubert, Mulvey \& Glasheen, 2011; Paxton, Valois, Watkins, Huebner \& Drane, 2007; McCarty, Stoep, Kuo, \& McCauley, 2006). Untreated depression is particularly concerning in youth due to the risk that it can lead to chronic medical conditions and shortened life span (Katon et al, 2010). Katon et al (2010) used a large population based study on adolescents in an integrated healthcare delivery system and revealed that youth with higher levels of depression had a poorer perception of their health, higher prevalence of academic challenges, strained relationships with family and peers and an increased likelihood of experimenting with substances. Early work by Deykin, Levy \& Wells (1987) found that a diagnosis of major depressive disorder was 34 times more common in students with a history of substance use compared to control subjects who denied using substances. Similar to these findings, a literature review of community studies related to substance use revealed that $60 \%$ of youth with substance use also had a secondary diagnosis of conduct disorder, ADHD or depression (Armstrong $\&$ Costello, 2002). Those who identified themselves as having more depressive symptoms were more likely to smoke, use alcohol at least weekly and report a lifetime use of substances. 
Symptoms of depression may also alter the course of substance use, a study by McKenzie, Olsson, Jorm, Romaniuk \& Patton (2010) found that adolescents who experienced high level of depression and smoked tobacco had more than a three-fold risk of nicotine dependence in young adulthood compared to youth who reported less than daily smoking. Earlier work by Kelder et al (2001) concluded that students who had the most depressive symptoms were 13.5 times more like for cocaine use, 9.1 times more likely for tobacco use, 8.7 times more likely for binge drinking and 6.1 times more likely for marijuana use. Adolescent substance use is significantly associated with an increased risk of substance use and dependence in later adulthood (Brook, Brook, Zhang, Cohen \& Whiteman, 2002). Depressed adolescents with inadequate support networks may use substances as a means to self-medicate, cope with psychological stressors and regulate feelings of depression (Kandel, Raveis \& Davies, 1991). A study conducted by Way, Stauber, Nakkula \& London (1993) used both a qualitative and quantitative method to explore the relationship between substance use and depression in two culturally diverse school environments (urban and suburban). Some youth described substance use as a means to "escape" and "relax" while youth who reported more depressive symptoms identified substance use as an added stressor. Students who attended schools in the suburban community reported being influenced by peers to use alcohol, tobacco and marijuana while none of the depressed youth attending urban schools reported being influenced.

\section{The Impact of Substance Use on Functioning}

The use of alcohol, tobacco and other drugs has a substantial impact on academic performance in adolescence. Research suggests that there are short and longer-term 
effects of drug use that impact one's ability to learn. Due to poor concentration, lack of attention, focus and drive grades tend to ultimately suffer as well as students losing an interest in participation in extra-curricular and pro-social activities. Studies consistently have found that grades, educational expectations, social disengagement and school misbehaviors are all positively correlated with alcohol, tobacco and other drug use (Patrick \& Schulenberg, 2014; Patrick \& Schulenberg, 2011, Patrick et al, 2011). If substance use is not identified and these concerns are not adequately addressed it can lead to truancy and school drop out. A study conducted by Hanson, Medina, Padula, Tapert, \& Brown (2011) examined cognition in a group of adolescents with alcohol and substance use history. The results revealed that cumulative effects of substance use and withdrawal symptoms during mid to late adolescence and into young adulthood related to deficits in cognitive functioning particularly related to visuospatial construction, executive function and visual and verbal memory. In addition to this, in all cases more use or withdrawal symptoms over the follow up period was associated with poorer neurophysiological functioning. Though the reasons to indulge in substance use may change across adolescence and into young adulthood. Understanding the motivation to initiate substance use may serve as a precursor to the development of other problematic behavioral problems and risky behaviors.

\section{Substance Use and Social Support}

\section{Parental Influence}

The role of family functioning has been investigated as both a correlate and predictor of adolescent substance use. Children from homes characterized by poor family functioning are at an increased risk for early substance initiation (Hummel, Shelton, 
Heron, Moore \& Van den Bree, 2012). Family management skills such as parental monitoring, discipline and structure reinforces good behavior. Parental monitoring lays a foundation for adolescent choices including peer selection and adolescent decisionmaking (Dever et al., 2012). Parental monitoring reflects the quality of the parent and child relationship and protects against adolescent substance use through positive supportive interactions and bonding (Schulenberg, Patrick, Maslowsky \& Maggs, 2014). Parenting includes discipline and setting rules regarding acceptable pro-social behavior. Although adolescents spend more time outside of the home with peers, parents still play a pivotal role in influencing adolescent behavior (Schulenberg et al., 2014). Research conducted by King and Chassin (2004) concluded that parental support was associated with 50\% less risk of developing a substance use disorder even after controlling for various risk factors. Adolescents who reside in non-supportive homes that are characterized by conflict between the parent and adolescent may be more likely to engage with deviant peers to gain social support and a sense of belonging (Hummel et al., 2012; Schulenberg et al., 2014). Adolescents may also engage in substance use as a means to cope with the hostility, lack of warmth or monitoring that may be present in their home (Hummel et al., 2012). A study by Simantov, Schoen \& Klein (2006) used a nationally representative survey that found that youth who reported high parental monitoring and support were approximately half as likely to smoke and drink as their peers who reported having less support and infrequent communication with their parents. Similar to these findings, a study by Dever et al (2012) examined the extent to which parental monitoring, school bonding, and sports participation protected against alcohol and marijuana use among adolescents who were identified as high risk takers. Results suggest that 
regardless of grade, gender, and risk-taking level, parental monitoring had the greatest impact on alcohol and marijuana use, thus indicating that parental involvement acts as a protective factor against substance use.

\section{Peer Influence}

Adolescence reflects a developmental stage when youth begin to develop autonomy and independence from their parents and spend more time outside of the home with peers. Characteristics of peer relationships provide adolescents with the opportunity to develop their pro-social skills and learn how to manage pressure from peers that may encourage them to engage in deviant behavior. Adolescent's attitudes regarding the acceptability and health risk associated with drug use are important factors that can impact their initiation of substance use (Tucker, Pollard, De la Haye, Kennedy \& Green, 2013). Youth may also perceive other social or personal benefits from substance use that overrides any identified adverse health outcomes. Developmental factors such as a sense of invulnerability and immortality coupled with a propensity to engage in risk taking behaviors influence the pattern of drug use among peers (Greydanus \& Patel, 2005). Peers serve as a key source of exposure to models of behavior that may promote alcohol, tobacco and marijuana use (Vogel, Rees, McCuddy \& Carson, 2015). When drug use is modeled, reinforced, and accepted by their peer group, youth are more likely to emulate this behavior and believe that it is acceptable and does not pose any negative health outcomes (Wright, Fagan \& Pinchevsky, 2014; Fagan \& Wright, 2012). A study conducted by Cleveland et al (2008) found that peer influence was a particularly strong risk factor for students in the $10^{\text {th }}$ and $12^{\text {th }}$ grade especially for lifetime use of alcohol and marijuana and these findings supported the notion that deviant peers influences the early 
initiation and ongoing use of substances. Later research conducted by Tucker et al (2013) found that adolescents were more likely to initiate marijuana use and binge drinking if a higher proportion of their peers engaged in alcohol and drug use.

Schools serve as a primary agent of socialization where youth can formulate relationships and engage with peers. Schools also provide an environment where youth establish their social status in peer groups. If adolescents perceive a connection between social status and behavior, any change in popularity may influence the initiation or frequency of substance use. A study conducted by Moody, Brynildsen, Osgood, Feinberg \& Gest (2010) used a longitudinal data set from the Promoting School-Community University Partnerships to Enhance Resilience (PROSPER) peers project to describe the linkages between popularity trajectory and substance use. Findings suggests that students with higher popularity were more likely to use substances even when controlling for past substance use. In addition to this, youth who moved in and out of high social status also engaged in more substance use that supports the notion that youth may adjust their substance use in an attempt to regain prior social status.

Schools also serve as a social control mechanism that inhibit adolescents from engaging in drug and alcohol use (Vogel, Rees, McCuddy, \& Carson, 2015). Misconducts tends to occur more readily in less socially cohesive schools where emphasis on academic performance is absent (Vogel et al., 2015). Conversely, high levels of school connectedness are associated with lower rates of drug alcohol and tobacco use (Vogel \& Barton, 2013). Vogel et al (2015) examined the influence of school connectedness and school drug culture on the association between peer network status and marijuana use. Results indicated that the effect of popularity of marijuana use was 
weaker among youth who attended highly connected schools. Social cohesion and connectedness to teachers may encourage students to refrain from engaging in behavior that would negative impact their image or relationship with their teachers or peers.

\section{Directions for Future Research}

In conclusion, adolescence is a critical developmental stage where adolescents seek new experiences, take risks and strive to identify and define their identity. While the adolescent brain continues to develop it is particularly vulnerable to the effects of alcohol, tobacco and marijuana use (Feinstein, Richter \& Foster, 2012; Schubert, Mulvey \& Glasheen, 2011). Since people are more likely to initiate tobacco, alcohol and other drug use during adolescence, early identification is critical in order to prevent the risk of future complications (U.S Department of Health and Human Services, Substance Abuse and Mental Health Services Administration, 2011; Anthony \& Petronis, 1995). According to the Monitoring the Future National Survey Results, by senior year, almost $67 \%$ of youth will have tried alcohol, $31 \%$ will have smoked a cigarette and $49.1 \%$ will have experimented with illicit drugs, marijuana accounts for almost $45 \%$ (Johnston, O’Malley, Miech, Bachman \& Schulenberg, 2016). Factors such as neighborhood conditions, school, parents and peers can all influence whether an adolescent will use alcohol, tobacco or drugs (Assink et al., 2015; Arthur et al., 2007). Substance use poses serious social and health risks that impacts an adolescent's ability to function. School failure, social conflict with parents and peers, mental health problems, sexual risk and involvement with the juvenile justice system as just a few consequences associated with substance use. It is critical that healthcare providers adequately screen adolescent patients for substance use problems in order to identify and treat those at risk or identified as 
having a substance use problem. More research is needed to evaluate the effectiveness of prevention programs in addition to early identification and treatment that is tailored to youth. Current literature has focused primarily on structural factors; however, more information is needed to better understand the contextual influences that affect adolescent substance use. Effective prevention programs should target multiple domains (community, peers and family) in order to better address the ways that each influence adolescent substance use collectively and individually. 


\section{Chapter III -Research Design \& Methods}

This chapter discussed the research methodology including the design, setting, sample, procedure, measurement instruments, data and statistical analysis for this study. The chapter focused on procedures for conducting research and protection of human subjects. This study is a secondary analysis of data from the Ohio Behavioral Health Juvenile Justice (BHJJ) initiative, a diversion program that is intended to address behavioral health and substance use issues in adolescents that are involved in the juvenile justice system.

\section{Design}

The purpose of this descriptive, correlational study was to examine the relationships amongst neighborhood disorganization, social support, substance use (ever used alcohol, tobacco and marijuana), depressive symptoms and functioning in a sample of adolescents involved in the Ohio behavioral health juvenile justice initiative. The study examined whether social support mediated the relationship between neighborhood disorganization and functioning as well as neighborhood disorganization and substance use behavior including type of substances ever used (alcohol, tobacco or marijuana). In addition to this, the study examined whether there were differences in social support and functioning based on age of first use (before or after the age of 12). Ultimately, the study tested a model controlling for age, race and gender that identifed whether neighborhood disorganization, social support, substance use (alcohol, tobacco and marijuana) or depressive symptoms were predictors of functioning.

Pearson-product moment correlation coefficient, independent samples t-test and regression analysis were used to test the proposed research questions. To date, no study 
has examined this set of variables in a population of adolescents involved in the juvenile justice system. This study examined the relationships between the variables depicted below in Figure 6. This descriptive correlational design was selected because it was the most appropriate approach to study the phenomenon of interest. This design is efficient and allowed the researcher to test complex relationships between multiple variables while controlling for potential covariates thus providing a foundation for future intervention studies. It also allowed one to determine the strength and direction of the various relationships. One disadvantage to this type of study design is that a correlation does not indicate causation, however since the purpose of this proposal was to examine potentially predictive and not causal relationships between the variables this study design was still ideal (Lobiondo-Wood, 2002).

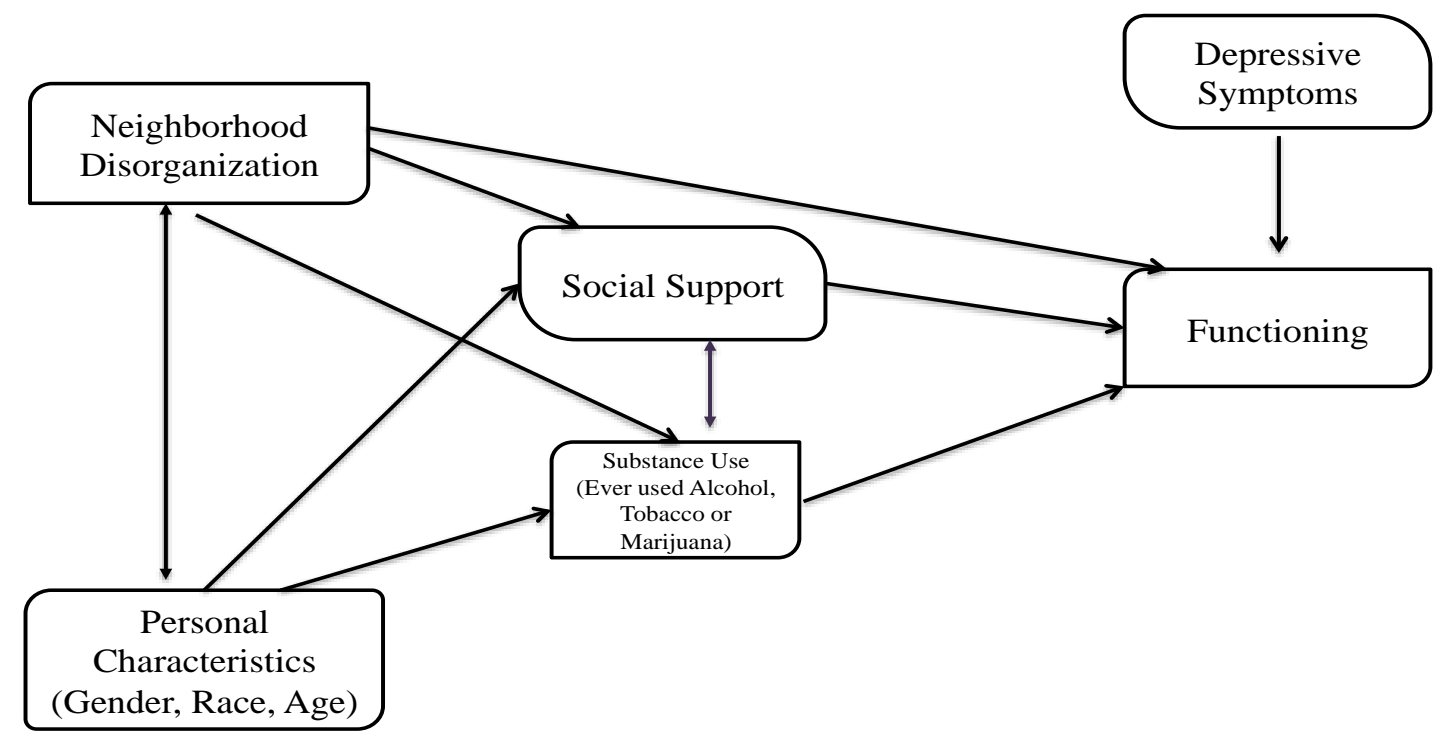

Figure 6: Conceptual Model 


\section{Research Questions}

This study addressed the following research questions:

(Q1a-d)- What are the relationships between (a) neighborhood disorganization, (b) social

support (peer and parental), (c) substance use (alcohol, tobacco and marijuana) and (d)

overall functioning in juveniles involved in the behavioral health juvenile justice initiative?

(Q2a-b)-Are there differences in neighborhood disorganization, social support and functioning by (a) gender (b) race and (c) level of depressive symptoms?

(Q3)- Are there differences in social support and functioning by age of first drug use (before or after age 12)?

(Q4a-b) What are the predictors of functioning in youth involved in the Behavioral Health Juvenile Justice Initiative controlling for age, race and gender? (b) What is the path model for the independent and dependent study variables on functioning?

(Q5) Does social support mediate the relationship between (a) neighborhood disorganization and substance use (alcohol, tobacco and marijuana) and (b) neighborhood disorganization and functioning?

\section{Setting}

The setting encompasses urban and rural counties that have participated in the Ohio BHJJ program since 2006. These counties include: Butler, Champaign, Cuyahoga, Fairfield, Franklin, Hamilton, Holmes, Logan, Lorain, Lucas, Mahoning, Montgomery, Summit, Trumbull, Union, Wayne and Wood. Table 3.1 depicted below provides information regarding the years that each county participated in the BHJJ initiative as well as the total number of youth enrolled in each county. Types of treatment models 
provided for each country was also listed in Table 1 . Counties are able to implement different protocols and procedures involving the juveniles into the program, inclusion criteria for eligibility and courses of their treatment plan. Detailed descriptions of seventeen counties protocols are listed in Table 3.1 and are also made available in the BHJJ evaluation report conducted by the Begun Center for Violence Prevention Research and Education, Mandel School of Applied Social Sciences of Case Western Reserve University.

Table 1. Protocols Implemented by County

\begin{tabular}{|c|c|c|}
\hline County (Sample size) & Participation Dates & Type of Protocol Implemented \\
\hline Butler $(n=28)$ & $2008-2009$ & Information unavailable \\
\hline Champaign $(n=97)^{*}$ & 2006-2009 & Information unavailable \\
\hline Cuyahoga $(n=371)^{*}$ & 2006-present & ICT, MST \\
\hline Fairfield $(n=32) *$ & 2006-2009 & Information unavailable \\
\hline Franklin $(n=470)^{*}$ & 2006-present & FFT, ICT, MDFT, MST \\
\hline Hamilton $(n=225)$ & 2008-present & FFT, A-CRA, Teen intervene \\
\hline Holmes $(n=11)$ & 2013-present & MST \\
\hline $\operatorname{Logan}(n=269)^{*}$ & 2006-2009 & Information unavailable \\
\hline Lorain $(n=35)$ & 2013-present & ICT \\
\hline Lucas $(n=218)$ & 2009-present & MST \\
\hline Mahoning $(n=23)$ & 2013-present & MST, TIP, high fidelity wraparound \\
\hline Montgomery $(n=1,420)^{*}$ & 2006-present & FFT, FFT-CM, Seven Challenges \\
\hline Summit $(n=223)$ & 2009-present & ICT, TF-CBT \\
\hline Trumbull $(n=21)$ & 2013-present & MST, TIP and high fidelity wraparound \\
\hline Union $(\mathrm{n}=31)^{*}$ & 2006-2009 & Information unavailable \\
\hline Wayne $(n=17)$ & 2013-present & MST \\
\hline Wood $(n=4)$ & 2013-2015 & FFT \\
\hline Total $(n=3,495)$ & ------------ & -------------- \\
\hline
\end{tabular}

Total of Youth Enrollment As of June 30, 2015

*Counties included in the subsample analysis

\section{Protocol Codes}

A-CRA=Adolescent Community Reinforcement Approach

FFT $=$ Functional Family Therapy

FFT-CM= Functional Family Therapy- Contingency Management

ICT $=$ Integrated Co-occurring Treatment

MDFT = Multidimensional Family Therapy

MST $=$ Multisystemic Therapy

TF-CBT $=$ Trauma-Focused Cognitive Behavioral Therapy

TIP=Transition to Independence Process 


\section{Sample}

This study analyzed data derived from the Ohio Behavioral Health Juvenile Justice (BHJJ) initiative, a diversionary program for youth 10-18 years of age involved in the juvenile justice system who also have a current DSM mental health diagnosis and substantial impairment in behavioral, cognitive and affective functioning (www.mhs.ohio.gov). A majority of these youth have co-occurring substance use, a history of violence and trauma exposure. These adolescents may also have a multi-system history, which is involvement in the juvenile justice and child welfare services (Kretschmar, Butcher \& Flannery, 2013). These youth are provided a psychological assessment upon entry in order to determine eligibility for the diversion program criteria and determine individualized evidence-based interventions that are appropriate (www.mhs.ohio.gov).

As of June 30, 2015 there were 3,495 youth enrolled in the BHJJ program with the average age of enrollment being 15.6 years. Not all juveniles that are involved in the justice system in Ohio will participate in the BHJJ diversion program; therefore information on the adolescents involved in the juvenile justice system who are not enrolled in the BHJJ program is not available in the evaluation of the sample. However, the study maintains information regarding reasons for termination from BHJJ services prior to successful completion. In the sample, 2,136 (61.4\%) were male and 1,341 (38.6\%) were female, with Caucasians representing $48.1 \%$ of the sample, African Americans representing $40.7 \%$ and $11.2 \%$ being represented by other racial groups. At intake, the majority of these youth, 1,885 (58.4\%), lived with their biological mother. DSM-IV Axis 1 diagnoses was reported at intake in the BHJJ program. A total of 7,599 
Axis diagnoses were identified for 3,342 youth, which equates to a mean of 2.27 diagnoses per youth. Over $43 \%$ of males and $35.2 \%$ of females were identified as having both a DSM-IV Axis 1 mental health diagnosis and a substance use diagnosis.

\section{Procedure for conducting research}

Prior to analyzing the BHJJ data, an application for approval to conduct the secondary analysis was submitted to the Institutional Review Board (IRB) at Case Western Reserve University (CWRU). In addition to the IRB approval, verbal approval was obtained from the Ohio Department of Youth Service (DYS) and the Ohio Department of Mental Health and Addiction Services (OhioMHAS). After approval was received from these sources, the data was analyzed via the Statistical Package for Social Sciences (SPSS) Version 24.

\section{Protection of Human Subjects}

The main purpose of the Institutional Review Board (IRB) is to review research projects in order to ensure that ethical standards are met and the rights of human subjects are protected. Informed consent was obtained from each participant's guardian if they are under the age of 18 and assent was obtained from each adolescent participant. The written assent ensures the adolescent expressed interest in participating, understands the purpose of the research study, and is fully aware of the potential risks and benefits associated with participation.

This project was a secondary analysis of the Ohio Behavioral Health Juvenile Justice Initiative data that was collected from 2006 until 2007, focusing specifically on data collected at the time of entry into the program. Since this is a secondary data analysis, there were no direct foreseeable risk to any of the participants involved in the 
study and all information on the participants has been de-identified. This study was focused on data collected on adolescents involved in the juvenile justice system who have been diagnosed with a mental health disorder, and no direct contact will be made with the participant for the purpose of this study (Kretschmar, Butcher \& Flannery, 2013).

\section{Measurement Instruments}

\section{Demographic Information}

Demographic information was obtained at the time of enrollment via surveys that included information on the date of enrollment, age, gender, race, custody arrangement and risk for out of home placement. Since a small percentage of Asians, Hispanics and Native Americans involved in the BHJJ initiative, the variable race was classified as white and non-white.

\section{Neighborhood Disorganization}

Neighborhood disorganization was defined at the zipcode level using the 2010 U.S Census data focusing entirely on the Ohio reported zipcodes. The seven observed measures for neighborhood disorganization included: percentage of female headed households with children under 18, percentage of residents with less than a high school education, percentage of renter occupied housing, percentage of residents who were unemployed, percentage of residents using supplemental security income, percentage receiving cash public assistance and the percentage of families below the poverty line. There is a percentage available via the U.S Census for each of the seven observed measures by zipcode and each of the percentages were converted to a z-score that was summed to obtain a final score for level of neighborhood disorder (Sampson \& Groves, 1989). Higher scores indicate greater neighborhood disorder while lower scores indicates 
that there is less disorder in that area relative to other participants in the sample. The zscore method has been validated in research as a valid tool to examine neighborhood disorganization (Dulin-Keita, Casazza, Fernandez, Goran \& Gower, 2013; Caughy, Nettles \& O’Campo, 2008; Drewnowski, Rehm \& Solet, 2007)

\section{Social Support}

In this study, social support identified the degree to which an adolescent is integrated in a social network and identifies family, friends or adult figures that are actively involved in their life (Cutrona, 2000). This form of support is defined as "emotional support" and includes acts of empathy, listening and generally "being there" for someone when needed or in times of distress (Cutrona, 2000). Social support was classified as both peer and parental support and was measured via questions derived from the Youth Information Questionnaire (YIQ). Three questions ask the adolescent their perception regarding whether or not they have an adult present in their life they can rely on for support and assistance, three questions asks the same question pertaining to their perception of peer support. Items from each subscale were summed separately and provided information related to the youth's perception of both peer and parental support. An Exploratory Factor Analysis (EFA) as well as a Cronbach's Alpha for both subscales tested internal consistency and reliability. This study examined whether social support had a mediating effect between (a) neighborhood disorganization and substance use (b) neighborhood disorganization and functioning.

\section{Substance Use}

Substance-Use was measured using the Substance-Use Survey-Revised that quantifies information on the onset, type and incidence of drug use (marijuana, cocaine, 
painkillers, etc). This study focused specifically on whether the adolescent had ever used alcohol, tobacco or marijuana and was coded as either "yes" or "no". The study also determined whether initiating substance use before the age of 13 had an impact on functioning and social support.

\section{Depressive Symptoms}

Depressive Symptoms were measured via the Trauma Symptom Checklist for Children (TSCC), and youth were classified as either "depressed" or "non-depressed". The TSCC is a self-report measure of children ages 8-16 and consists of 54 items that yields two validity scales (hyper-response and under-response) and six clinical scales. Each item is rated on a 4-point likert scale with 0 "never" and 3 "almost all of the time". Raw scores are converted into standard scales, and T scores that are above 65 are considered clinically significant outcomes. T scores that are within the range of 60-65 represent subclinical symptomology and were included in the "depressed" group for this analysis while those below a score of 60 were classified as "non-depressed".

\section{Function}

Functioning was measured from the Ohio Youth Problem, Functioning and Satisfaction Scale that was designed to assess clinical outcomes for children and adolescents with severe emotional and behavioral disorders (Ogles, Melendez, Davis \& Lunnen, 2001). This scale defines functioning in a variety of daily activities including interpersonal relationships, recreation, self-direction and motivation. This measure assesses four primary domains of outcomes with four subscales: Problem Severity, Functioning, Hopefulness, and Satisfaction with services. This project used the 20 -item likert functioning scale that ranges from zero "not at all" to five "all the time" in the last 
30 days. A total score is obtained by summing all of the items on the scale based on the social worker's report of how the youth functions. The Ohio Youth Problem, Functioning and Satisfaction Scale demonstrated acceptable internal consistency and reliability (Dowell \& Ogles 2008).

\section{Data Collection}

Data collection for the BHJJ program began in 2006 and continues on an ongoing basis as more youth are added to the program. BHJJ youth are administered surveys at intake, discharge and during regular intervals throughout the program. This study focused specifically on the data that is collected at the time of entry into the program. Youth are provided a packet of surveys by the program staff that are completed by hand, electronically scanned into a database, and manually checked using the Teleform software for inaccuracies. Teleform is a software program that allows for data transmission via fax, scanner or PDF file (Kretschmar, Butcher \& Flannery, 2013). Trained evaluators conduct data entry, and each participating youth is assigned a unique identification number that protects their anonymity. Coded items for each instrument is entered into a SPSS data file and checked for errors and consistency by a trained assistant.

\section{External and Internal Validity}

Threats to Internal Validity. Internal validity referred to the rigor of the study and determined whether an experimental treatment or condition affected an outcome (Briggs, 2008). Therefore, a threat to internal validity compromised one's confidence to conclude that there is a causal relationship between the independent and dependent variables (Fraenkel \& Wallen, 2009; Briggs, 2008). In this study, the threat related to selection 
must be considered and minimized. Random selection typically minimizes this threat; however, considering the nature of this study it would be unethical to randomly select which youth can and can not receive an intervention that has demonstrated successful results (Kretschmar, Butcher \& Flannery, 2013).

Threats to External Validity. External validity referred to the generalizability of the outcomes to various settings and populations (Briggs, 2008; Ferguson, 2004, Cook \& Campbell, 1979). External validity can be classified into two categories, population and ecological validity. Population validity is a threat to one's ability to generalize the study results to a larger population of interest (Fraenkel \& Wallen, 2009; Ferguson, 2004; Cook \& Campbell, 1979). Ecological validity focused on the conditions within the research setting that can influence findings. Campbell \& Stanley (1963) identified four factors that threaten external validity based on study conditions which include: (a) interactional effects of testing (b) interactional effects of selection biases with experimental arrangements (c) reactive effects of experimental arrangements and (d) multiple treatment interference. Threats to external validity included threats related to population validity secondary to the use of a convenience sample, which has the potential to introduce bias and not result in a representative sample. Therefore, generalizability to other populations must be considered with caution.

\section{Data Management}

BHJJ data is password protected and original questionnaires are locked in a secure location at the Begun Center for Violence Prevention Research and Education, Mandel School of Applied Social Sciences, Case Western Reserve University. Jeff Kretschmar $\mathrm{PhD}$ is the principal investigator for this study and oversees the research and evaluation 
services. The Ohio Department of Mental Health and Addiction Services (OhioMHAS)

and the Ohio Department of Youth Services (DYS) work in collaboration with the research team and provide permission for the data to be used for outside analysis.

\section{Statistical Analysis}

This section detailed how the researcher addressed the proposed research questions and analysis plan for the study. The first question used Pearson-product moment correlation coefficient/correlation matrix to examine the relationships between (a) neighborhood disorganization, (b) social support, (c) substance use (ever used alcohol, tobacco or marijuana) and overall functioning in juveniles involved in the behavioral health juvenile justice initiative. If there is evidence of a relationship the project also determined the strength and direction of the relationship. The second and third research question used an Independent samples t-test to determine the mean differences between groups. Question two examined whether there were differences in neighborhood disorganization, social support and functioning by gender, race and depressive symptoms and question three examined whether there were differences in social support and functioning by age of first drug use being before or after the age of 12. Part A of the fourth question used a hierarchical regression analysis to determine the predictors of functioning and part B used a regression analysis to determine the path model. The last question tested whether social support mediated the relationship between neighborhood disorganization and substance use and neighborhood disorganization and functioning. A mediation model is one that seeks to identify and explain the process that underlies an observed relationship between an independent variable and a dependent variable via the inclusion of a third variable known as the mediator. A variable acts as a mediator when 
variation in an independent variable accounts for variations in the proposed mediator (path a), variations in the dependent variable (path b) and when both paths are controlled, there is no longer a significant relationship between the independent and dependent variable (path c). According to Baron \& Kenny (1986) the strongest evidence of mediation is when this path is zero.

\section{Assumptions for Statistical Analysis}

After approval was received from the Case Western Reserve University IRB, Ohio Department of Youth Service (DYS) and Ohio Department of Mental Health and Addiction Services (OhioMHAS), the data was cleaned using the Statistical Package for Social Sciences (SPSS) version 24. In order to proceed with each statistical analysis, certain assumptions were evaluated for each statistical test. In order to carry out the Pearson's product moment correlation coefficient, independent samples t-test and regression analysis the following four assumptions were evaluated. These included:

(1) The variables must be continuous and be measured at the interval or ratio level. (Corty, 2007)

(2) There also needs to be a linear relationship between the variables. Plotting the dependent variable against the independent variable on a scatterplot allows one to visually inspect the scatterplot for linearity. If the relationship in the scatterplot is non-linear, the data can either be transformed or an equivalent non-parametric tests can be used (Corty, 2007).

(3) There should be no significant outliers in the data that can impact the findings. One can detect outliers by visual inspection of the scatterplot and identifying extreme values. If outliers have been identified from the scatterplot, the Cook's 
distance statistic will determine if the outlier influence the model. If the Cook's distance value is greater than 1.0 , the outlier is considered influential and needs to be removed from the analysis (Corty, 2007; Tabachnick \& Fidell, 2007).

(4) The variables should also be normally distributed and this assumption can be tested with the Shapiro-Wilk tests of normality. The null hypothesis is that the data are normally distributed therefore if the alpha $=0.05$ and the $p$-value is less than 0.05 then the null hypothesis that the data are not normally distributed is rejected (Corty, 2007).

(5) For regression analysis, the assumption of homoscedasticity implies that the variance of the residuals is constant for all levels of the independent variable (Corty, 2007; Tabachnick \& Fidell, 2007). This assumption is analyzed by plotting the standardized residuals against the predicted dependent variable and checking for an even spread around the zero line.

\section{Evaluating the Assumptions for Statistical Analysis}

Descriptive statistics were used to report frequencies and variability of the variables. Normality of each variable was determined by analysis of the skewness and kurtosis, central tendency (mean, mode and median) and dispersion of scores (range, variance, standard deviation). Influential cases (outliers) are identified by Cook's D values and visually observing the frequencies and partial plots. Visual inspection of the histogram, normal Q-Q plot, detrended Q-Q plot and boxplot also assists to determine the skewness and kurtosis for each variable. Values for skewness should fall between 3 and 3 and 8 and -8 for kurtosis (Corty, 2007). If the data is determined to be positively or 
negatively skewed or kurtotic it should be transformed by one of the various acceptable methods and retested for skewness and kurtosis (Tabachnick \& Fidell, 2007).

In order to determine whether there are differences in neighborhood disorganization, social support, substance use and functioning by (a) gender (b) race and (c) depressive symptoms, the independent samples t-test will be utilized. This test will also be used to determine whether there are differences in social support and functioning based on age of first use being before or after the age of 12 . This statistical test examines whether the means of two independent groups are statistically different from one another. The assumptions for the tests are similar to the Pearson product moment correlation coefficient and regression analysis.

Hierarchical multiple regression allows you to add sets of variables to a regression equation and determine how much each set of variables uniquely adds to the prediction of the dependent variable, usually expressed as the increase in $R^{2}$ (the variance explained in the dependent variable). These sets are added to the regression equation in a fixed, sequential order determined by theoretical, logical and empirical evidence. The regression analysis will be used to determine the predictors of functioning in addition to evaluating whether social support is a mediator.

In addition to the assumptions previously mentioned, for the hierarchical multiple regression it is also important to determine whether the assumptions of multicollinearity has been violated. This assumption is measured in multiple ways and requires that independent variables not be highly correlated $>0.80$. Tolerance measures the influence of one independent variable on all other independent variables, and the $\mathrm{T}$ values must be $>.10$ to ensure that no multicollinearity is present. The Variance Inflation Factor (VIF) 
assesses the strength of the linear relationship between a specific predictor variable and the other predictors also measures this assumption with scores $>10$ indicating that multicollinearity might be present. Lastly, the condition index that is found in the collinearity diagnostic should not be greater than 15 otherwise it could be an indication that multicollinearity is present. A variable causing multicollinearity should be removed from the analysis if the concept measured by that variable is theoretically measured by another variable.

The visual examination of the residual plots of standardized residuals versus predicted values determined whether the assumption of linearity was met (Mertler \& Vannatta, 2005). If this assumption is not met, as evidenced by a curvilinear pattern, transformation of the independent variables or dependent variable is recommended prior to rerunning the analysis (Mertler \& Vannata, 2005). The Durbin-Watson statistic should be between 1.5 and 2.5 and test whether there is autocorrelation in the residuals. This is a critical assumption that is not robust to violation.

\section{Summary}

In summary, this was a descriptive correlational study designed to test the relationships between neighborhood disorganization, social support (peer and parental), substance use (ever used alcohol, tobacco and marijuana), depressive symptoms and functioning in a group of youth involved in the Behavioral Health Juvenile Justice Initiative. A predictive correlational design was used to test the relationships between the variables and determine the predictors of functioning, controlling for age, race and gender. The analysis included descriptive statistics, demographic information as well as a series of correlation matrix and regression analyses. The mediation roles of social support 
was examined in addition to a pathway model being created that illustrated the path of each independent variable on the outcome variable functioning. 


\section{Chapter IV-Results}

This study was a secondary data analysis of data collected during the first two years of the Behavioral Health Juvenile Justice Initiative, an Ohio diversion program created to enhance services for juvenile offenders with serious behavioral health needs. This chapter summarizes the results and provides an interpretation of the findings.

The purpose of this descriptive exploratory study was to examine the relationship amongst neighborhood disorganization, social support, substance use (alcohol, tobacco and marijuana), depressive symptoms and functioning in a sample of youth involved in the Ohio Behavioral Health Juvenile Justice Initiative. This project also assessed whether these variables differed by race, gender and depressive symptoms. The study determined whether individual or multiple factors function together or individually to predict overall functioning. Lastly, it evaluated whether social support mediated the relationship between neighborhood disorganization and functioning and neighborhood disorganization and substance use.

\section{Description of Study Location}

The Ohio Behavioral Health Juvenile Justice Initiative services youth ages 10-18 with a DSM diagnosis and significant impairment in behavioral, cognitive and/or affective domains. A majority of the youth entering the program have co-occurring substance abuse and a history of violence, exposure to trauma and criminal behavior. Approximately 3,500 youth have been enrolled in the program from 2006-2016. This analysis focuses on a subset of the youth who enrolled in the program from 2006 to 2007 and completed the Youth Information Questionnaire (YIQ), providing information related to their perceptions of parental and peer social support. Since 2006, 17 urban, suburban 
and rural counties in the state of Ohio had been selected to participate in the program.

Table 4.1 illustrates the number of youth who resided in each of the 7 counties that compose this sample.

Table 2. Youth Location by County $(n=408)$

\begin{tabular}{lll}
\cline { 1 - 2 } County & Number of Youth Enrolled & Percentage \\
\cline { 2 - 3 } Cuyahoga & 30 & $7.4 \%$ \\
Fairfield & 9 & $2.2 \%$ \\
Franklin & 134 & $32.8 \%$ \\
Logan & 132 & $32.4 \%$ \\
Montgomery & 48 & $11.8 \%$ \\
Union & 16 & $3.9 \%$ \\
Champaign & 39 & $9.6 \%$ \\
Total & 408 & $100 \%$ \\
\hline
\end{tabular}

\section{Demographic Characteristics of Study Participants}

This subset of 408 adolescents represents 96 zipcodes, of these $52 \%(n=212)$ of the sample resided in an urban area and $48 \%(n=196)$ of the sample resided in a rural area. Table 2 provides a description of the youth who completed the YIQ questionnaire and compose the sample. The majority of the sample was male $(52.9 \%, \mathrm{n}=216)$ and white $(63.5 \%, \mathrm{n}=259)$, with a mean age of 15 years old. The majority of the youth resided with their biological or adoptive parents $(79.9 \%, n=326)$, and over half $(53.2 \%, n=217)$ of the sample were at risk for being placed out of the home due to their delinquent behaviors and court involvement. 


\begin{tabular}{|c|c|c|}
\hline Variable & $\underline{\mathrm{n}}$ & $\underline{\%}$ \\
\hline \multicolumn{3}{|l|}{$\overline{\text { Gender }}$} \\
\hline Male & 216 & $52.9 \%$ \\
\hline Female & 192 & $47.1 \%$ \\
\hline \multicolumn{3}{|l|}{ Race } \\
\hline White & 259 & $63.5 \%$ \\
\hline Non-white & 149 & $36.5 \%$ \\
\hline \multicolumn{3}{|l|}{ Age of the Youth } \\
\hline $8-10$ years & 4 & $1.0 \%$ \\
\hline 11-13 years & 49 & $12.0 \%$ \\
\hline 14-16 years & 262 & $64.2 \%$ \\
\hline $17-18$ years & 93 & $22.8 \%$ \\
\hline \multicolumn{3}{|l|}{ Custody Arrangement } \\
\hline Biological Parents & 326 & $79.9 \%$ \\
\hline Adoptive/Stepparent & 23 & $5.6 \%$ \\
\hline Foster Parent & 2 & $0.5 \%$ \\
\hline Sibling (Biological, Step) & 1 & $0.2 \%$ \\
\hline Aunt or Uncle & 10 & $2.5 \%$ \\
\hline Grandparent & 32 & $7.8 \%$ \\
\hline Other Family Relative & 2 & $0.5 \%$ \\
\hline Friend & 2 & $0.5 \%$ \\
\hline Other & 6 & $1.5 \%$ \\
\hline Missing & 4 & $1.0 \%$ \\
\hline \multicolumn{3}{|l|}{ Risk of Out of Home Placement } \\
\hline Yes & 217 & $53.2 \%$ \\
\hline No & 62 & $15.2 \%$ \\
\hline Missing & 129 & $31.6 \%$ \\
\hline
\end{tabular}

\section{Description of Study Variables}

\section{Independent Variables}

The independent variables in this study include: Neighborhood disorganization, social support (perceptions of peer and parental support), substance use (ever used alcohol, tobacco and marijuana), and depressive symptoms.

Neighborhood disorganization. This variable was defined at the zipcode level using the 2010 U.S Census data focusing entirely on the Ohio reported zipcodes. The seven 
observed measures for neighborhood disorganization included: percentage of female headed households with children under 18, percentage of residents with less than a high school education, percentage of renter occupied housing, percentage of residents who were unemployed, percentage of residents using supplemental security income, percentage receiving cash public assistance and the percentage of families below the poverty line. Each percentage was converted into a $\mathrm{z}$-score that was summed to create a final composite score for level of neighborhood disorganization. This technique has been validated and used in previous research studies such as Sampson \& Morenoff (2004).

For the neighborhood disorganization variable, an exploratory factor analysis was completed to test the internal consistency and reliability of the measure. Prior to examining the internal consistency and reliability of the neighborhood disorganization variable, a Pearson correlation coefficient for each measure was examined ensuring that there were no correlations $<0.3$ and $>0.85$. This step is necessary in order to avoid concerns related to items in the scale being too highly correlated and increasing the risk of redundancy or the items not being correlated enough to suggest a relationship is present (Fields, 2009; Tabachnick \& Fidell, 2007). Correlations were significant at the 0.01 level (2-tailed) with correlations ranging from 0.55 to 0.83 .

An Exploratory Factor Analysis (EFA) was conducted on the 7 neighborhood disorganization items to evaluate parsimony and describe the maximum amount of common variance using the smallest number of explanatory constructs (Field, 2009). There are visual and analytical approaches to determining the number of factors to extract that includes observation of the scree plot and identification of Eigenvalues greater than one. For the neighborhood disorganization variable all 7 items loaded to one factor with 
factor loadings being greater than 0.78 . According to Fields (2009), factors that have four or more loadings with values that are greater than 0.6 are considered reliable regardless of the sample size (Fields, 2009).

The Kaiser-Meyer-Olkin (KMO) measured the sampling adequacy and proportion of variance explained for this factor analysis. Values should fall between 0 to 1 with values closer to 1 indicating that the pattern of correlations are compact and should yield distinct and reliable factors. The KMO was 0.90 , which falls within the range of "great" according to Fields (2009).

Lastly, to evaluate internal consistency a Cronbach's Alpha was run which measured the scale reliability and explained how closely related a set of items were as a group. The Cronbach's Alpha for the neighborhood disorganization variable based on 7 items was $\alpha=0.87$ which is an indicator of "good" internal consistency (Fields, 2009). Social Support. Social support was measured using the Youth Information Questionnaire (YIQ) that asks youth questions related to youths' perceptions of peer and parental support (Kretschmar, Butcher \& Flannery, 2013). To address concerns related to multicollinearity between the items, the Pearson correlation coefficients for parental and peer social support was examined ensuring that there were correlations between 0.3 and 0.85 . Correlations were significant at the 0.01 level (2-tailed) with correlations ranging from $0.50-0.70$ for parental support and 0.47-0.64 for peer support.

An Exploratory Factor Analysis (EFA) was conducted on the social support questions and visual inspection of the scree plot suggested a two-factor solution, thus separating the scale into parental and peer support. For the parental and peer social 
support variable all factor loadings were greater than 0.5 , which is considered "reliable" regardless of sample size (Fields, 2009).

The Kaiser-Meyer-Olkin (KMO) measured the sampling adequacy and proportion of variance explained for this factor analysis. Values should fall between 0 to 1 with values closer to 1 indicating that the pattern of correlations are compact and should yield distinct and reliable factors. The $\mathrm{KMO}$ (peers) $=0.669$ and the $\mathrm{KMO}($ parental $)=0.673$ which falls above the acceptable range of 0.5 (Fields, 2009).

The Cronbach's Alpha for the parental social support variable was $\alpha=0.81$ and the Cronbach's Alpha for peer support was $\alpha=0.77$, which are both acceptable values for internal consistency (Fields, 2009).

Substance use. The substance use scale is a youth self-report measure of whether the adolescent has ever used alcohol, tobacco or marijuana. Each substance is reported and measured as either "yes" or "no" for each substance. The study also examined the impact of age of first substance use being before or after the age of 12 .

Depressive Symptoms. Depressive symptoms were measured using the Trauma Symptom Checklist for Children (TSCC), and youth were classified as either "depressed" or "non-depressed" (Briere, 1996). The TSCC is a self-report measure of depressive symptoms for children aged 8-16 and consists of 54 items that yields two validity scales (hyper-response and under-response) and six clinical scales. Each item is rated on a 4point likert scale with 0 "never" and 3 "almost all of the time". Raw scores are converted into standard scales, and $\mathrm{T}$ scores that are above 65 are considered clinically significant for depressive symptoms. $\mathrm{T}$ scores that are within the range of 60-65 represent subclinical depressive symptomology but were included in the "depressed" group for this analysis, 
while those below a score of 60 were classified as "non-depressed." The TSCC clinical scales have demonstrated good internal consistency and reliability with Cronbach's alpha ranging from .81 to .88 .

Function. Functioning was measured using the 20 item functioning scale of the Ohio Youth Problems, Functioning and Satisfaction scale (Ogles, Melendez, Davis \& Lunnen, 2001). For the measure, workers rated the youth's level of functioning on a variety of daily activity areas such as interpersonal relationships, recreation, self-direction and motivation. Each item is rated on a five-point scale ( 0 "extreme troubles" to 4 "doing very well"). Higher scores are indicative of better functioning. The Ohio Youth Problem, Functioning and Satisfaction Scale has been used in previous research demonstrating excellent internal consistency and reliability, $\alpha=0.94$ (Dowell \& Ogles, 2008).

\section{Preliminary Data Screening}

\section{Normality of Study Variables}

Table 4 displays the values for skewness and kurtosis for the independent and dependent variables. These variables fell within the recommended values for larger samples sizes of -8 to 8 for skewness and -20 to 20 for kurtosis (Tabachnick \& Fidell, 2007). Examination of the histograms, boxplots and Q-Q plots also indicated the data points were normally distributed. The central limit theorem also states that in sample sizes greater than 50 , most of the sample means will be close to the population mean and will demonstrate normal distribution. 


\begin{tabular}{lcccc}
\hline \multicolumn{1}{c}{ Variable } & Mean & $\frac{\text { Standard }}{\frac{\text { Deviation }}{(\text { SD })}}$ & $\underline{\text { Kkewness }}$ & $\underline{\text { Kurtosis }}$ \\
$\begin{array}{lcccc}\text { Neighborhood } \\
\text { Disorganization }\end{array}$ & 0 & & $0.79+/-.12$ & $.47+/-.24$ \\
& & & & \\
$\quad \begin{array}{l}\text { Social Support } \\
\quad \text { Parental }\end{array}$ & 13.0 & 4.02 & $-.59+/-.12$ & $-.55+/-.24$ \\
$\quad$ Peer & 12.7 & 4.05 & $-.51+/-.12$ & $-.60+/-.24$ \\
& & & & \\
Substance Use & .68 & .47 & $-0.76+/-.12$ & $-1.43+/-.24$ \\
$\quad$ Alcohol & .67 & .47 & $-0.73+/-.12$ & $-1.47+/-.24$ \\
$\quad$ Tobacco & .68 & .46 & $-0.78+/-.12$ & $-1.40+/-.24$ \\
$\quad$ Marijuana & & & & \\
Depression & 5.94 & 23.29 & $3.76+/-.12$ & $12.22+/-.24$ \\
Functioning & 42.51 & 14.00 & $-.18+/-.12$ & $-.56+/-.24$ \\
\hline
\end{tabular}

* Indicates significant value, $\mathrm{p}<.05$

\section{Missing data}

This sample $(\mathrm{N}=408)$ represents the youth who completed the YIQ Questionnaire in 2006-2007 and did not have any missing data for the study variables. For each of the variables that were included in the analysis, youth who had missing values were removed prior to analysis. Although there was complete data for age of first substance use being before or after the age of 12 , there was missing data for age of first use by age groupings for alcohol, tobacco and marijuana. Although, this information is included in the descriptive statistics, age of first use was not analyzed by age groupings therefore the missing values did not impact the analysis. Table 5 provides information related to the descriptive statistics for age of alcohol, tobacco and marijuana use separated by age groupings and age of first use being before or after the age of 12 . 


\begin{tabular}{|c|c|c|}
\hline $\begin{array}{l}\frac{\text { Variable }}{\text { Age of First Use }(<12} \\
\text { years })\end{array}$ & $\underline{\mathrm{n}}$ & $\underline{\%}$ \\
\hline Yes & 178 & $43.6 \%$ \\
\hline No & 230 & $56.4 \%$ \\
\hline
\end{tabular}

Age of First Use

\begin{tabular}{lcc}
$\quad$ Alcohol & 2 & \\
<8 years & 18 & $0.6 \%$ \\
8-10 years & 125 & $4.4 \%$ \\
11-13 years & 127 & $30.6 \%$ \\
14-16 years & 3 & $31.1 \%$ \\
$>17$ years & 133 & $0.7 \%$ \\
Missing & & $32.6 \%$ \\
$\quad$ Tobacco & & \\
<8 years & 13 & $3.2 \%$ \\
$8-10$ years & 16 & $3.9 \%$ \\
$11-13$ years & 62 & $15.2 \%$ \\
$14-16$ years & 27 & $6.6 \%$ \\
$>17$ years & --- & $0 \%$ \\
Missing & 290 & $71.1 \%$ \\
$\quad$ Marijuana & & \\
$<8$ years & & \\
8-10 years & 2 & $0.5 \%$ \\
11-13 years & 6 & $1.5 \%$ \\
$14-16$ years & 58 & $14.2 \%$ \\
$>17$ years & 46 & $11.3 \%$ \\
Missing & --- & $0 \%$ \\
\hline
\end{tabular}

Research Question 1. What are the relationships between (a) neighborhood disorganization, (b) social support (peer and parental), (c) substance use (ever used alcohol, tobacco and marijuana) and (d) overall functioning in a sample of youth involved in the behavioral health juvenile justice initiative?

\section{Assumptions for Pearson r}

This question was answered using the Pearson product moment correlation. There are four assumptions that underpin a Pearson's correlation and they were tested prior to 
running the analysis (Fields, 2009; Mertler \& Vannatta, 2005). The first assumption is that the variables must be measured at the continuous level. Each of the independent and dependent variables that were included in the correlation were measured at the ratio level, thus not violating this assumption. The second assumption is the requirement for a linear relationship between the variables. This assumption was checked by visually inspecting the scatterplots to check for linearity, and there were no concerns noted. The third assumption states that there should be no significant outliers in the sample. Potential outliers were examined by noting values that had a $\mathrm{z}$ score greater than +3.00 or less than -3.00 (Mertler \& Vannatta, 2005), in addition to visual inspection of box plots and histograms which suggested there were no significant outliers in the sample. The last assumption states that the variables should be normally distributed and shows homoscedasticity. Homoscedasticity means the variance of the residuals should be the same for all predicted scores (Tabachnick and Fidell, 2007). This assumption was checked by looking at scatterplots between $\mathrm{X}$ and $\mathrm{Y}$ variables, and it was not violated. 


\section{Findings}

Table 6. Correlations among Study Variables $(\mathrm{N}=408)$

\begin{tabular}{|c|c|c|c|c|c|c|c|}
\hline Variable & ND & Parental SS & Peer SS & ETOH ever & Cig ever & MJ ever & Func \\
\hline ND & 1 & -.04 & -.07 & -.09 & -.10 & .06 & $-.15^{* *}$ \\
\hline Parental SS & -.05 & 1 & $.30^{* *}$ & .02 & $.13^{* *}$ & -.01 & $.27^{* *}$ \\
\hline Peer SS & -.06 & $.30^{* *}$ & 1 & $.11^{*}$ & $.10^{*}$ & .06 & $.13^{* *}$ \\
\hline ETOH ever & -.08 & .02 & $.11^{*}$ & 1 & $.45^{* *}$ & $.56^{* *}$ & .02 \\
\hline Cig ever & $-.11^{*}$ & $.13^{* *}$ & $.10^{*}$ & $.45^{* *}$ & 1 & $.42^{* *}$ & .05 \\
\hline MJ ever & .09 & .01 & .06 & $.56^{* *}$ & $.42^{* *}$ & 1 & $-.11^{*}$ \\
\hline Func & $-.21^{* *}$ & $.27^{* *}$ & $.13^{* *}$ & .03 & .05 & $-.11^{*}$ & 1 \\
\hline
\end{tabular}

* Correlation is significant at the 0.05 level (2-tailed)

** Correlation is significant at the 0.01 level (2-tailed)

Note.

$\mathrm{ND}=$ Neighborhood Disorganization

Parental SS= Parental Social Support

Peer SS= Peer Social Support

ETOH ever= Ever used alcohol

Cig ever $=$ Ever used cigarettes

MJ ever=Ever used alcohol

Func $=$ Youth functioning (Social worker report)

The Pearson product moment correlation coefficient matrix were examined to determine if any significant relationships existed between neighborhood disorganization, parental and peer social support, substance use and functioning which is illustrated in Table 6.

Neighborhood Disorganization. There was a significant negative correlation between neighborhood disorganization and youth functioning $(r=-.15, \mathrm{p}<0.01)$, suggesting that youth residing in more disorganized environments demonstrated more impairment with functioning.

Social Support. Parental social support had a significant positive relationship with peer social support $(\mathrm{r}=0.30, \mathrm{p}<0.01)$, cigarette use $(\mathrm{r}=0.14, \mathrm{p}<.01)$ and functioning $(\mathrm{r}=0.28$, $\mathrm{p}<0.01)$. Peer social support was positively correlated with alcohol use $(\mathrm{r}=0.11, \mathrm{p}<0.05)$, cigarette use $(r=0.10, p<0.05)$ and functioning $(r=0.13, p<0.01)$. This suggests that youth who identify having parental social support also had a positive relationship with peers. In 
addition to this, youth who reported having peer social support reported more alcohol use than youth who did not have peer social support. Lastly, youth who identified having parental and peer social support also were more likely to report trying cigarettes and had overall improved functioning compared to youth who reported less parental and peer support.

Substance Use. There was a positive relationship between ever trying alcohol and cigarettes $(\mathrm{r}=0.45, \mathrm{p}<0.01)$ and ever trying alcohol and marijuana $(\mathrm{r}=0.56, \mathrm{p}<0.01)$. This suggests that youth who have tried alcohol are likely to have tried cigarettes and marijuana. There was also a significant positive relationship between cigarette use and marijuana use $(r=0.42, p<0.01)$. Suggesting that those who have tried cigarettes are likely to have tried marijuana.

Research Question 2a-2c: Are there differences in neighborhood disorganization, social support and functioning based on (a) gender (b) race and (c) depressive symptoms?

\section{Assumptions for Independent Samples t-test}

This question was answered using the independent samples t-test. There are four assumptions that underpin an independent samples t-test. The first two assumptions were not violated and state that each observation within the sample is independent of another and the dependent variable is interval or ratio level. The third assumption states that the two populations are from normally distributed samples, which was reviewed earlier with the Pearson $r$ correlation and was not violated. The last assumption states that the variables demonstrate homogeneity of variance, meaning the two populations from which the samples are selected must have equal variances. Observing the significance of the Levene's test for a $\mathrm{p}$ value $<0.05$ checked this assumption. A significant Levene's test 
rejects the null hypothesis is rejected and indicates that the test results include an adjustment for the lack equal variances.

Gender. The Independent Samples t-test reports the difference between two independent means. Gender was dichotomized into male (0) and female (1). For gender, neighborhood disorganization and parental social support were both significant $(\mathrm{p}<.05)$. Since the Levene's test for equality of variances was significant for both parental social support and neighborhood disorganization we rejected the null hypothesis that the variances of the two groups are equal to one another. Males reported higher levels of parental social support than females and the difference was significant $\mathrm{t}(362.77)=2.59$, $\mathrm{p}<.05$. Females reported higher levels of neighborhood disorganization than males and the differences were significant $\mathrm{t}(406)=-3.19, \mathrm{p}<.05$.

There were no statistically significant findings for peer social support and functioning by gender. Table 7 illustrates the means, $95 \%$ confidence intervals and pvalues for the variables. 
Table 7. Independent Samples t-test: Gender

\begin{tabular}{|c|c|c|c|c|}
\hline$\underline{\text { Variable }}$ & $\underline{\text { Mean }}$ & $\underline{\mathrm{t} \text {-value }}$ & $\underline{95 \% \mathrm{CI}}$ & p-value \\
\hline \multicolumn{5}{|c|}{ Neighborhood Disorganization } \\
\hline Female $^{\mathrm{a}}$ & $1.00(6.43)$ & -3.19 & $-3.06-(-0.73)$ & $\mathrm{p}=0.00 *$ \\
\hline Male $^{\mathrm{b}}$ & $-0.89(5.49)$ & & & \\
\hline \multicolumn{5}{|c|}{ Parental Support } \\
\hline Female $^{\mathrm{a}}$ & $12.40(4.46)$ & 2.59 & $.025-1.83$ & $\mathrm{p}=0.01 *$ \\
\hline Male $^{\mathrm{b}}$ & $13.44(3.52)$ & & & \\
\hline \multicolumn{5}{|l|}{ Peer Support } \\
\hline Female $^{\mathrm{a}}$ & $12.53(4.14)$ & 0.81 & $-.46-1.11$ & $\mathrm{p}=0.42$ \\
\hline Male $^{\mathrm{b}}$ & $12.86(4.00)$ & & & \\
\hline \multicolumn{5}{|l|}{ Functioning } \\
\hline Female $^{\mathrm{a}}$ & $42.86(13.8)$ & -0.47 & $-3.38-2.08$ & $\mathrm{p}=0.64$ \\
\hline Male ${ }^{b}$ & $42.21(14.2)$ & & & \\
\hline $\begin{array}{l}* \mathrm{p}<.05 \text { lev } \\
\text { CI=Confide } \\
{ }^{\mathrm{a}} \mathrm{n}=192 \\
{ }^{\mathrm{b}} \mathrm{n}=216 \\
\mathrm{a}=\text { female } \\
\mathrm{b}=\text { male }\end{array}$ & & & & \\
\hline
\end{tabular}

Race. Race was dichotomized into non-white (0) and white (1). For race, neighborhood disorganization, parental social support and functioning were all significant $(\mathrm{p}<.05)$. Since the Levene's test for equality of variances was significant for neighborhood disorganization we reject the null hypothesis that the variances of the two groups are equal to one another and read the equal variances not assumed line to report findings. Therefore, non-whites reported higher levels of neighborhood disorganization than whites, and the difference was significant $\mathrm{t}(237.92)=9.25, \mathrm{p}<.05$. The Levene test for equality of variances was non-significant for parental social support and functioning so we will fail to reject the null hypothesis that the variances of the two groups are equal to one another and will read the equal variances assumed line to report findings. Parental social support was significant therefore whites reported greater social support than non- 
whites, $\mathrm{t}(406)=-2.33, \mathrm{p}<.05$ and whites reported higher functioning than non-whites, $\mathrm{t}$ $(406)=-4.87, \mathrm{p}<.05$.

There were no significant findings for peer social support by race. Table 8

illustrates the means, $95 \%$ confidence intervals and p-values for the variables.

Table 8. Independent Samples t-test: Racial Differences

Variable $\underline{\text { Mean }} \underline{\text { t-value }} \underline{95 \% \mathrm{CI}}$ p-value

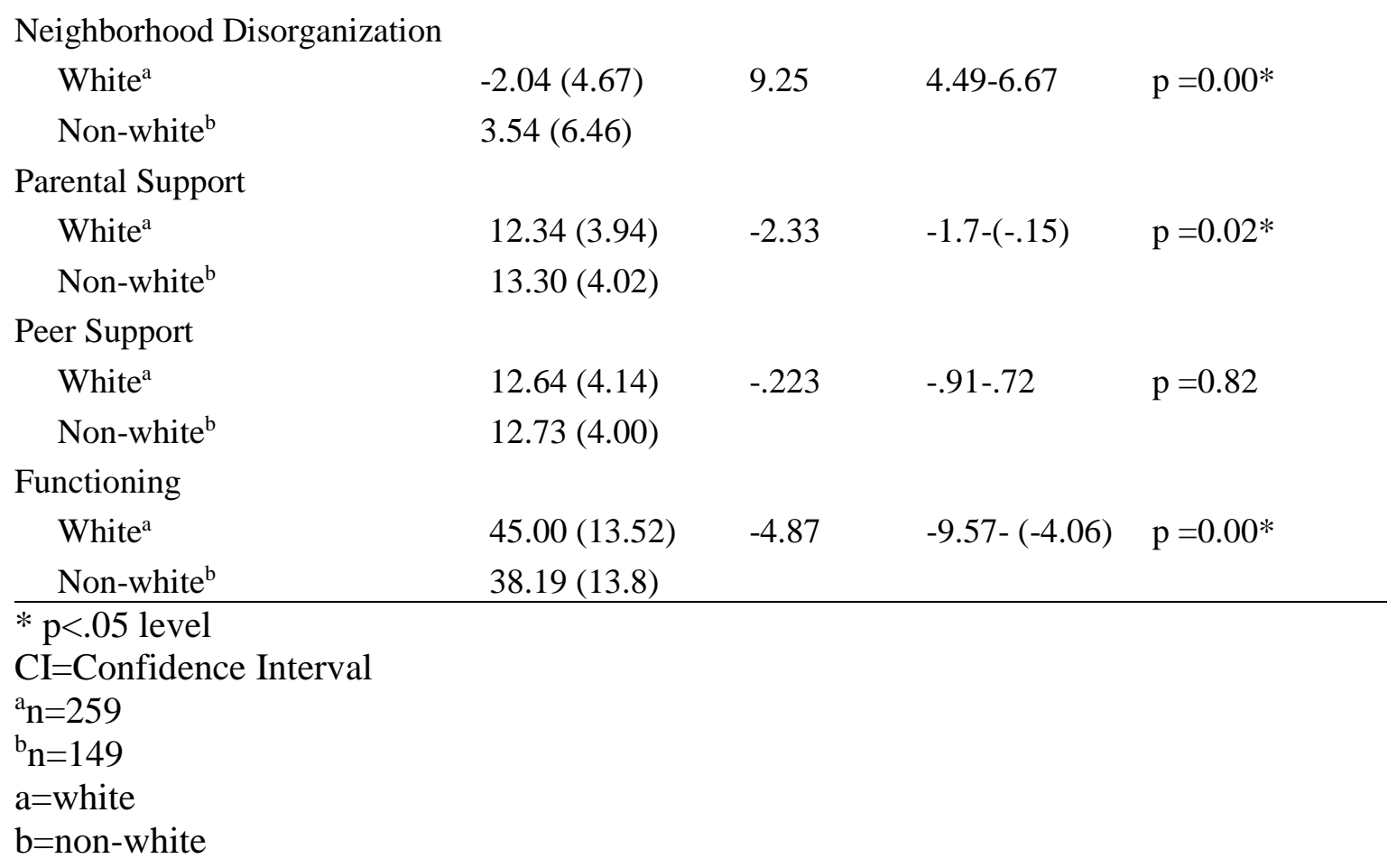

Depressive symptoms. Depressive symptoms were dichotomized into depressed (1) and non-depressed (0). For depressive symptoms, neighborhood disorganization, peer social support and functioning were all significant $(\mathrm{p}<.05)$. Since the Levene's test for equality of variances was significant for neighborhood disorganization we will reject the null hypothesis that the variances of the two groups are equal to one another and read the equal variances not assumed line to report findings. Therefore, non-depressed youth reported higher levels of neighborhood disorganization than depressed youth and this 
difference was significant $\mathrm{t}(71.98)=2.37, \mathrm{p}<.05$. For peer social support and functioning the Levene's test for equality of variances was not significant so we fail to reject the null hypothesis that the variances of the two groups are equal to one another and we will report the equal variances assumed line. Therefore, youth with depressive symptoms reported less social support than youth without depressive symptoms $t(382)=2.32, p<.05$ and youth with depressive symptoms also reported lower functioning $\mathrm{t}(382)=2.31, \mathrm{p}<.05$ than youth who reported less depressive symptoms.

There were no significant findings for parental social support by depressive symptoms, although there was a trend toward significance $(\mathrm{P}=.06)$. Table 9 illustrates the means, $95 \%$ confidence intervals and $p$-values for the variables.

Table 9. Independent Samples t-test: Depressive Symptoms

\begin{tabular}{|c|c|c|c|c|}
\hline Variable & Mean & $\underline{\mathrm{t} \text {-value }}$ & $95 \% \mathrm{CI}$ & p-value \\
\hline \multicolumn{5}{|c|}{ Neighborhood Disorganization } \\
\hline Non-depressed ${ }^{\mathrm{a}}$ & $0.28(6.16)$ & 2.37 & $0.29-3.40$ & $\mathrm{p}=0.02 *$ \\
\hline Depressed $^{\mathrm{b}}$ & $-1.57(4.92)$ & & & \\
\hline \multicolumn{5}{|l|}{ Parental Support } \\
\hline Non-depressed $^{\mathrm{a}}$ & $13.11(3.95)$ & 1.82 & $-.08-2.34$ & $\mathrm{p}=0.07$ \\
\hline Depressed $^{\mathrm{b}}$ & $11.98(4.66)$ & & & \\
\hline \multicolumn{5}{|l|}{ Peer Support } \\
\hline Non-depressed ${ }^{\mathrm{a}}$ & $12.86(4.12)$ & 2.32 & $.22-2.68$ & $\mathrm{p}=0.02 *$ \\
\hline Depressed $^{\mathrm{b}}$ & $11.40(3.82)$ & & & \\
\hline \multicolumn{5}{|l|}{ Functioning } \\
\hline Non-depressed ${ }^{\mathrm{a}}$ & $43.30(13.85)$ & 2.31 & $.72-9.06$ & $\mathrm{p}=0.02 *$ \\
\hline Depressed $^{\mathrm{b}}$ & $38.41(13.92)$ & & & \\
\hline \multicolumn{5}{|l|}{$* \mathrm{p}<.05$ level } \\
\hline $\begin{array}{l}C I=\text { Confidence In } \\
{ }^{a} n=335 \\
{ }^{b} n=49 \\
a=\text { non-depressed } \\
b=\text { depressed }\end{array}$ & & & & \\
\hline
\end{tabular}

Research Question 3: Are there differences in social support and functioning by age of first drug use (before or age after 12)? 
There were no statistical significance for parental social support, peer social support and functioning by age of substance use being before or after the age of 12 . However, p-values suggest a trend towards lower peer social support and functioning for youth who started substances before the age of 12 . Table 8 illustrates the mean's, $95 \%$ confidence intervals and $\mathrm{p}$-values for the variables.

\section{Table 10. Independent Samples t-test: Age of First Substance Use}

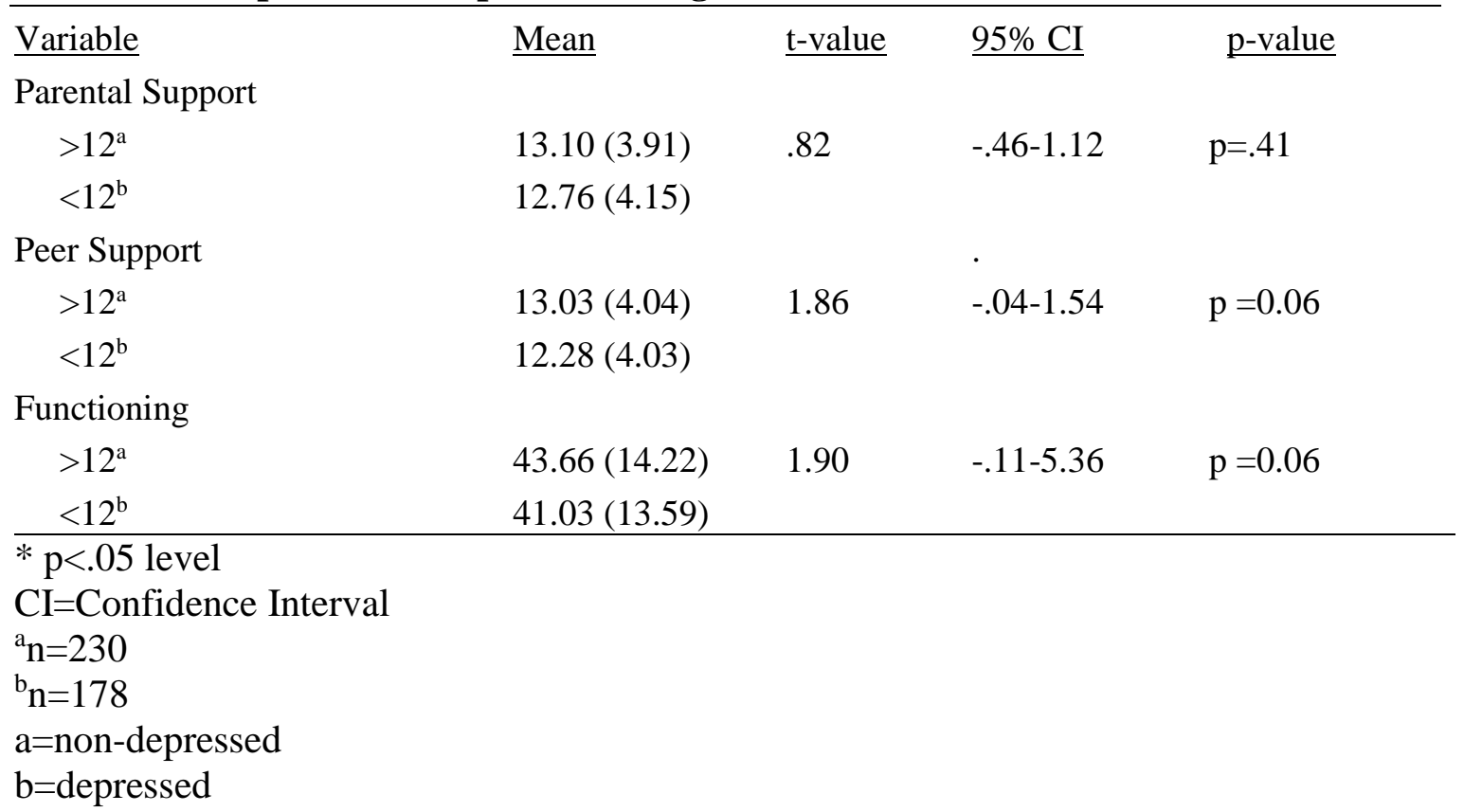

Research Question 4a: What are the predictors of functioning in youth involved in the Behavioral Health Juvenile Justice Initiative controlling for age, race and gender? A hierarchical multiple regression was conducted to examine the predictors of functioning controlling for age, gender and race. Assumptions to test for variance, linearity, absence of influential case, normality or residuals, constant error variance and multicollinearity were all assessed and fell within the acceptable ranges to proceed with analysis. The primary and secondary assumptions for hierarchical regression analysis included the following. 


\section{Primary \& Secondary Assumptions for Hierarchical Multiple Regression}

Linearity. The assumption of linearity requires that the association of the $\mathrm{X}$ variable and Y variable must be linear and when graphed follow a straight line (Tabachnick \& Fidell, 2001). Regression can accurately estimate the relationship between dependent and independent variables when the relationship is linear in nature. If the relationship between the dependent and independent variables is not linear, the results of the regression analysis will under or overestimate the true relationship and increase the risk of Type I and Type II errors (Tabachnick \& Fidell, 2001). The partial regression scatterplots were analyzed for each of the continuous study variables to test this assumption. The partial plots option assessed the effect of each independent variable on the dependent variable controlling for all of the other predictor variables. Comparing the linear and quadratic lines of best fit based on differences in $\mathrm{R}$ squares values were assessed to ensure that the differences did not exceed 1-2\% additional explained variance and no concerns were noted (Fields, 2009).

Homoscedasticity. This assumption requires that the distribution of a y score should have approximately an equal score on $\mathrm{x}$. Homoscedasticity was tested by examining the constant error variance and plotting the Studentized Deleted Residuals (y axis) against the Standard Predicted value (x axis) and determining whether the residuals are in an even, random scatter around the zero line. Values fell within the recommended range with "scatter" falling between a 3:1 ratio of highest to lowest error variance.

Normality. Regression analysis assumes that variables have normal distributions (Fields, 2009). This means that errors are normally distributed, and that a plot of the values of the residuals will approximate a normal distribution curve. Superimposing the normal curve 
and assessing the data plots, and p-plots for indications of skewness and kurtosis ensures that this assumption has not been violated. For larger sample sizes, values that are greater than -8 and 8 for skewness and -20 to 20 for kurtosis suggests that there may be concerns related to the normality of the residuals, however our values fell within the acceptable ranges and was less than -8 and 8 for skewness and -20 and 20 for kurtosis.

Multicollinearity. This assumption is measured in multiple ways and requires that independent variables not be highly correlated $>0.80$. Tolerance measures the influence of one independent variable on all other independent variables and the $\mathrm{T}$ values must be $>.10$ to ensure that no multicollinearity is present. Each of our values fell within the range of 0.77-0.99 thus indicating that there were no concerns with multicollinearity. The Variance Inflation Factor (VIF) also measured this assumption with scores greater that 10 indicating that multicollinearity might be present and the maximum value for this sample was 1.3.

Absence of influential cases. This assumption examined if all cases exert approximately the same influence on the regression coefficients. Influential cases are identified by looking at the Cook's D Residual Outliers table and there were no values in our sample which were greater than 1.0. The partial plots of the dependent variable by each independent variable were also assessed and labeled by standardized deleted residuals, which did not indicate there was a concern for any influential cases.

Independence of Observations. This assumption requires that independent observations were collected and there isn't any nested data or data collected at multiple time points on the same participant. This study only used results at one time point therefore this assumption was not violated. The Durbin Watson is also a test statistic used to test this 
assumption and ensure there is no autocorrelation in the residuals. The value for this sample was 1.9 which is between the acceptable range of $0-4$, and does not suggest that there are any concerns. 
Table 11. Multiple Regression Zero Order Correlation

\begin{tabular}{|c|c|c|c|c|c|c|c|c|c|c|c|}
\hline$\underline{\text { Variable }}$ & $\underline{\text { Age }}$ & $\underline{\text { Race }}$ & $\underline{\text { Gender }}$ & $\underline{\text { ND }}$ & $\underline{\text { Parent SS }}$ & $\underline{\text { Peer SS }}$ & $\underline{\text { ETOH }}$ & $\underline{\text { Cig }}$ & $\underline{\text { MJ }}$ & $\underline{\text { Dep }}$ & $\underline{\text { Func }}$ \\
\hline Age & 1.00 & -.03 & .00 & -.00 & .03 & .07 & $.32 * *$ & $.16^{*}$ & $.33^{* *}$ & $.13^{*}$ & .08 \\
\hline Race & -.03 & 1.00 & $-.09 *$ & $-.49 * *$ & $.11^{*}$ & .01 & $.14^{*}$ & $.24 * *$ & -.08 & .02 & $.23 * *$ \\
\hline Gender & .00 & $-.09 *$ & 1.00 & $.14^{*}$ & $-.13^{*}$ & -.04 & .03 & .07 & -.01 & -.05 & .02 \\
\hline ND & -.02 & $-.45 * *$ & $.16^{*}$ & 1.00 & -.04 & -.07 & -.09 & -.10 & .06 & -.03 & $-.15^{* *}$ \\
\hline Parent SS & .03 & $.11 *$ & $-.13 *$ & -.05 & 1.00 & $.30 * *$ & .02 & $.13^{*}$ & -.00 & -.02 & $.28 * *$ \\
\hline Peer SS & .07 & .01 & -.04 & -.06 & $.30 * *$ & 1.00 & $.11^{*}$ & $.10 *$ & .06 & .03 & $.13 * *$ \\
\hline ЕTOH & $.32 * *$ & $.14 * *$ & .03 & -.08 & .02 & $.11^{*}$ & 1.00 & $.45^{* *}$ & $.56^{* *}$ & .04 & .01 \\
\hline Cig & $.16^{*}$ & $.24 * *$ & -.07 & -.11 & $.13^{*}$ & $.10^{*}$ & $.45^{* *}$ & 1.00 & $.42 * *$ & .04 & .05 \\
\hline MJ & $.33^{* *}$ & $-.08 * *$ & .01 & $.09 *$ & -.00 & .06 & $.56^{* *}$ & $.42 * *$ & 1.00 & .06 & $-.10^{*}$ \\
\hline Dep & $.13 * *$ & .02 & -.05 & -.01 & -.02 & .03 & .04 & .04 & .06 & 1.00 & -.10 \\
\hline Func & .08 & $.24 * *$ & .02 & $-.21 * *$ & $.28 * *$ & $.13^{*}$ & .01 & .05 & $-.10^{*}$ & -.05 & 1.00 \\
\hline
\end{tabular}

Note

$* \mathrm{p}<.05 * * \mathrm{p}<.01$

Age $=$ Age in years Race $=$ White $=0 /$ Non - white $=1$

Gender $=$ Male $=0 /$ Female $=1$

$\mathrm{ND}=$ Neighborhood Disorganization

Parent SS= Parental Social Support Peer SS= Peer Social Support

ETOH $=$ Ever used alcohol Cig= Ever used tobacco MJ= Ever used marijuana

Dep $=$ Non-Depressed $=0 /$ Depressed $=1$

Func $=$ Youth functioning $($ Worker report $)$ 
Table 12. Summary of Hierarchical Regression Analysis for Functioning ( $N=408)$

\begin{tabular}{|c|c|c|c|c|}
\hline \multirow{2}{*}{ Predictor Variable } & \multicolumn{2}{|c|}{ Model A } & \multicolumn{2}{|c|}{ Model B } \\
\hline & $\mathrm{B}$ & $\beta$ & $\mathrm{B}$ & $\beta$ \\
\hline Constant & 25.21 & & 10.88 & \\
\hline Gender & 1.25 & 0.09 & 2.22 & 0.08 \\
\hline Race & 7.01 & $0.24 *$ & 5.22 & $0.18 *$ \\
\hline Age & 0.78 & 0.04 & 1.07 & $0.12 *$ \\
\hline Neighborhood Disorganization & & & -0.15 & -0.07 \\
\hline Ever used alcohol & & & 0.36 & 0.01 \\
\hline Ever used cigarettes & & & -0.35 & -0.01 \\
\hline Ever used marijuana & & & -3.80 & $-0.13^{*}$ \\
\hline Peer Social Support & & & 0.18 & 0.05 \\
\hline Parental Social Support & & & 0.85 & $0.24 *$ \\
\hline Depressive Symptoms & & & -0.03 & -0.05 \\
\hline $\mathrm{R}^{2}$ & 0.06 & & 0.15 & \\
\hline Adjusted $\mathrm{R}^{2}$ & 0.06 & & 0.13 & \\
\hline $\mathrm{F}$ & $9.31 *$ & & $7.18 *$ & \\
\hline
\end{tabular}

4a) First, the correlations table were examined to check for multicollinearity between the predictors, and there were no values greater than 0.8 (Table 11). The highest correlation in the sample was 0.56 , which fell within the acceptable range and did not demonstrate any concern that the assumption was violated. The Cook's distance was examined to ensure that the maximum value falls below 1.0 and our value was 0.02 , which indicates that there were no concerns for influential cases. To test for normality, the standard residuals were observed to ensure that the values fell between -3 and 3 and our max value was 2.64 and our minimum values was -2.91 , which is not indicative of any outliers. Observation of the Normal P-P plot revealed that the majority of the values fell along the line, which suggest the assumption of homoscedasticity was not violated. 
Model's A and model B were both significant with $\mathrm{p}<.01$ (Table 12). The ANOVA test was also significant $(\mathrm{p}<.05)$ for both model A and B which is important because it suggest that the slope of the line was zero. In model A, $6.5 \%$ of the variance in the dependent variable (functioning) was explained by race $(\beta=0.24, p<.01)$ and this equation was statistically significant $(\mathrm{F}=9.31, \mathrm{p}<.01)$. While in Model $\mathrm{B}, 15.3 \%(\mathrm{~F}=7.18$, $\mathrm{p}<.01)$ of the variance in the dependent variable was explained by four independent variables. The independent variable in the regression equation that was the strongest predictor was parental social support $(\beta=0.24, \mathrm{p}<.01)$, followed by race $(\beta=0.18, \mathrm{p}=0.01)$, marijuana use $(\beta=-0.13, p=0.05)$ and age $(\beta=0.12, p=0.02)$. In model $A$, race was statistically significant with $(\beta=0.24, \mathrm{p}=0.00)$. Therefore, when controlling for gender, race and age, an additional $8.8 \%$ of the variance in functioning was explained by parental social support and marijuana use, for a total explained variance of $21.8 \%$; $78.2 \%$ of the variability in functioning remains unexplained.

Research Question 4b) What is the final pathway model between each dependent and independent variable?

\section{Findings}

A series of regression models were run to determine the predictors for each of the study variables. Assumptions for the model were previously tested with question 4a and there were no concerns noted. For each regression, insignificant paths ( $p>.05)$ were removed from the analysis until the final model only contained significant paths. Figure 8 is an overview of the final pathway model with significant paths being illustrated along with their respected beta weights. 
Functioning. The first dependent outcome variable of interest was functioning, Figure 8 illustrates the four significant paths. These paths include: parental social support ( $\beta=.25$, $\mathrm{p}<.01)$, race $(\beta=.20, \mathrm{p}<.05)$, age $(\beta=.12, \mathrm{p}<.05)$, and marijuana use $(\beta=-.13, \mathrm{p}<.05)$. Findings suggest that $15.3 \%(\mathrm{~F}=7.18, \mathrm{p}<.05)$ of the variance in the dependent variable functioning was explained by the independent variables parental social support, race, age and marijuana use.

Depressive symptoms. Similarly, for depressive symptoms, all of the study variables were added to the multiple regression. Figure 8 illustrated the predictors of depressive symptoms which was age $(\beta=.13, \mathrm{p}<.05)$. Approximately $1.7 \%(\mathrm{~F}=7.08, \mathrm{p}<.05)$ of the variance in the dependent variable depressive symptoms was explained by the independent variable age.

Substance Use. For substance use, each independent variable was added to the multiple regression and Figure 8 illustrates the predictors of ever using alcohol, cigarette and marijuana. Predictors of alcohol use were age $(\beta=0.33 \mathrm{p}<.05)$ and race $(\beta=0.15, \mathrm{p}<.05)$. Approximately $12.7 \%(\mathrm{~F}=29.58, \mathrm{p}<.05)$ of the variance in the dependent variable alcohol use was explained by the independent variables age and race. For cigarette use, predictors were race $(\beta=0.25, \mathrm{p}<.05)$, age $(\beta=0.17, \mathrm{p}<.05)$ and gender $(\beta=0.10 \mathrm{p}<.05)$.

Approximately 9.5\% $(\mathrm{F}=14.10, \mathrm{p}<.05)$ of the variance in the dependent variable cigarette use was explained by the independent variables race, age and gender. Lastly, age ( $\beta$ $=0.33, \mathrm{p}<.05)$ was a predictor of marijuana use and approximately $10.7 \%(\mathrm{~F}=48.65$ $\mathrm{p}<.05$ ) of the variance in marijuana use was explained by the adolescent's age. Social Support. All of the variables were added to the multiple regression analysis. Figure 8 illustrated the predictors of parental social support which were cigarette use 
$(\beta=.14, \mathrm{p}<.05)$ and gender $(\beta=-.14, \mathrm{p}<.05)$. Approximately $13.0 \%(\mathrm{~F}=17.89, \mathrm{p}<.05)$ of the variance in the dependent variable parental support was explained by the independent variables cigarette use, gender and peer social support. Peer social support did not have any significant values as a predictor therefore; it was removed from the model.

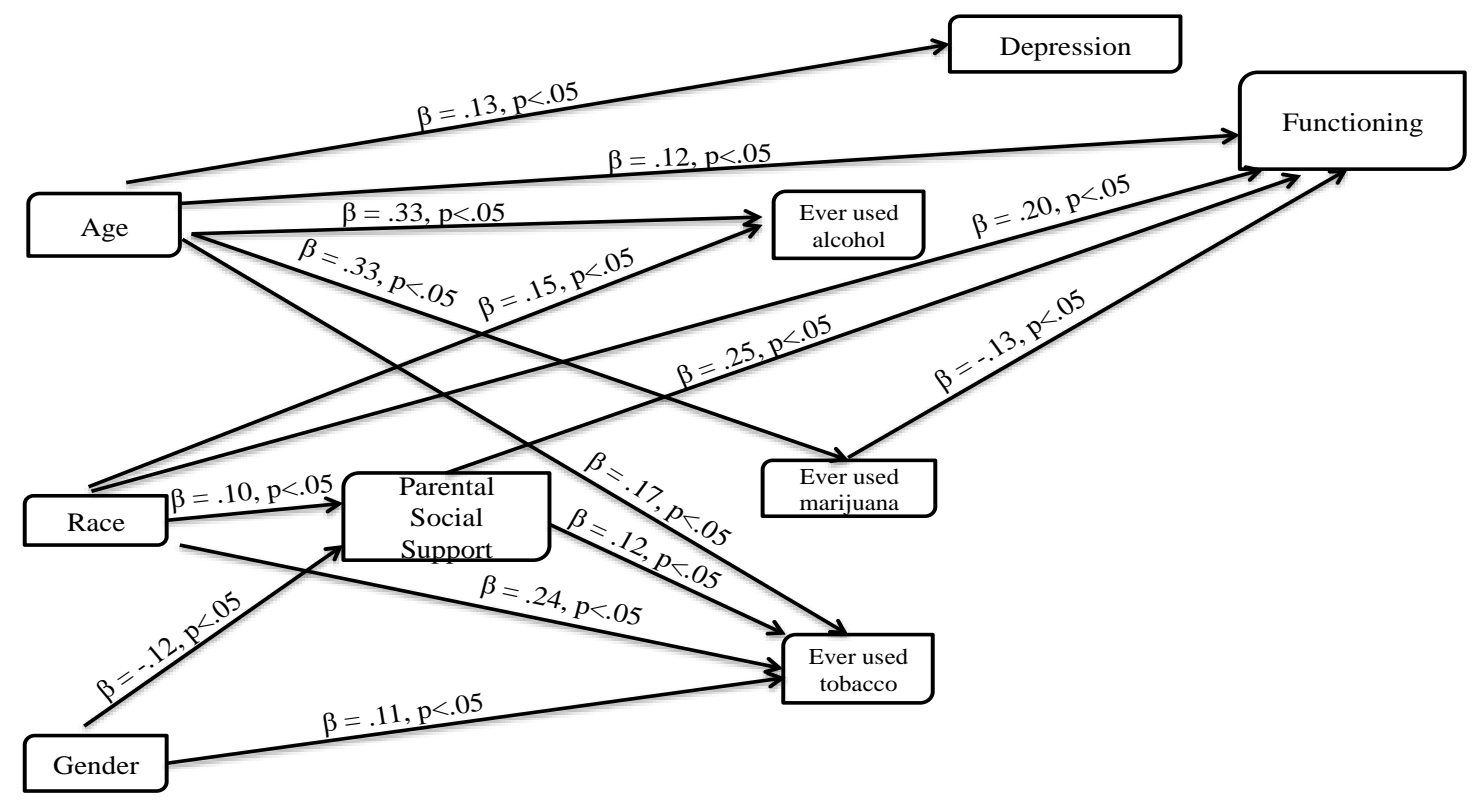

Figure 8. Final Pathway Model for Study Variables $(\mathrm{N}=408)$

Research Question 5: Does social support mediate the relationship between (a) neighborhood disorganization and functioning and (b) neighborhood disorganization and substance use (alcohol, tobacco and marijuana)?

Mediation occurs when variation in the independent variable accounts for variation in the mediator (path a), variation in the dependent variable (path b) and when path $\mathrm{a}$ and $\mathrm{b}$ are controlled the relationship between the independent and dependent variable is reduced or becomes non-significant (path c). The mediating effect of peer and 
parental social support between neighborhood disorganization and functioning was first examined. The goal of mediation analysis is to establish the extent to which some causal variable $\mathrm{X}$ influences an outcome $\mathrm{Y}$ through one or more mediator variables.

\section{Neighborhood Disorganization and Functioning}

The test for mediation effect of parental and peer social support between neighborhood disorganization and functioning was conducted as described by Baron and Kenney (1986). The Pearson product moment correlation table (Table 13) was examined to ensure necessary requirements for mediation to occur was met. In order for peer and parental social support to act as mediators there must be a significant correlation between neighborhood disorganization and functioning and since this requirement was met, mediation was examined via the SPSS add on macro PROCESS which generates direct and indirect effects in mediation models (Hayes, 2012).

\section{Table 13. Test of Support as Mediator between ND and Functioning}

\begin{tabular}{lcccc}
\hline Variable & $\underline{\text { ND }}$ & $\underline{\text { Peer SS }}$ & $\underline{\text { Parental SS }}$ & $\underline{\text { Func }}$ \\
ND & 1 & -.07 & -.04 & $-.15^{* *}$ \\
Peer SS & -.06 & 1 & $.30^{* *}$ & $.13^{* *}$ \\
Parental SS & -.05 & $.30^{* *}$ & 1 & $.27^{* *}$ \\
Func & $-.21^{* *}$ & $.13^{* *}$ & $.27^{* *}$ & 1 \\
\hline
\end{tabular}

$* \mathrm{p}<.0 .05$ level (2-tailed).

$* * p<0.01$ level (2-tailed).

\section{Findings}

For peer social support, paths a-c needed to be significant to illustrate that mediation had taken place. Path a from neighborhood disorganization to social support was found to be non-significant $F(1,406)=1.48, \mathrm{p}>.05, \mathrm{R}^{2}=.0036$. Path $\mathrm{b}$ from social support to functioning was significant $F(2,405)=12.29, \mathrm{p}<.05, \mathrm{R}^{2}=0.06$. Lastly, Path c from neighborhood disorganization to functioning had statistical significance $\mathrm{F}(1,406)=$ 
$18.49, \mathrm{p}<.05, \mathrm{R}^{2}=.04$. In addition to this, the confidence intervals were not statistically different from zero suggesting that peer social support is not a mediator between neighborhood disorganization and functioning.

For parental social support, paths a-c needed to be significant to illustrate that mediation had taken place and path a from neighborhood disorganization to social support was found to be non-significant $F(1,406)=1.20, p>.05, R^{2}=.0029$. Path $b$ from social support to functioning was statistically significant $\mathrm{F}(2,405)=26.00, \mathrm{p}<.05, \mathrm{R}^{2}=$ .11. Lastly, path c from neighborhood disorganization to functioning was found to be statistically significant $\mathrm{F}(1,406)=18.49, \mathrm{p}<.05, \mathrm{R}^{2}=.04$. Although there were significant $\mathrm{b}$ and $\mathrm{c}$ paths, $\mathrm{p}<0.05$ for parental and peer support, path a must also be significant in order for there to be a mediating effect. In addition to this, the confidence interval for both parental and peer social support were not statistically different from zero suggesting parental and peer support is not a mediator between neighborhood disorganizations and functioning.

\section{Neighborhood Disorganization and Substance Use}

The test for mediation effect of parental and peer social support between neighborhood disorganization and substance use was examined as described by Baron and Kenney (1986). The Pearson product moment correlation table (Table 14) was examined to ensure necessary requirements for mediation to occur was met. In order for peer and parental social support to act as mediators there must be a significant correlation between neighborhood disorganization and substance use. Since this requirement was not met, peer and parental social support could not serve as a mediator between neighborhood disorganization and ever using alcohol, tobacco or marijuana. 
Table 14. Test of Support as Mediator between ND and Substance Use

\begin{tabular}{|c|c|c|c|c|c|c|}
\hline$\underline{\text { Variable }}$ & $\underline{\text { ND }}$ & $\underline{\text { Peer SS }}$ & $\frac{\text { Parental }}{\underline{\mathrm{SS}}}$ & $\frac{\text { ETOH }}{\underline{\text { ever }}}$ & $\underline{\text { Cig ever }}$ & $\underline{\text { MJ ever }}$ \\
\hline ND & 1 & -.07 & -.04 & -.09 & -.10 & .06 \\
\hline Peer SS & -.06 & 1 & $.30^{* *}$ & $.11^{*}$ & $.10^{*}$ & .06 \\
\hline Parental SS & -.05 & $.30^{* *}$ & 1 & .02 & $.13^{* *}$ & -.01 \\
\hline ETOH ever & -.08 & $.11^{*}$ & .02 & 1 & $.45^{* *}$ & $.56^{* *}$ \\
\hline Cig ever & $-.11^{*}$ & $.10^{*}$ & $.13^{* *}$ & $.45^{* *}$ & 1 & $.42^{* *}$ \\
\hline MJ ever & .09 & .06 & -.00 & $.56^{* *}$ & $.42^{* *}$ & 1 \\
\hline
\end{tabular}

*. Correlation is significant at the 0.05 level (2-tailed).

**. Correlation is significant at the 0.01 level (2-tailed).

Note.

$\mathrm{ND}=$ Neighborhood Disorganization

\section{Findings}

Table 14 illustrates the Pearson product moment correlation table that illustrates that there is no correlation between neighborhood disorganization and alcohol, tobacco or marijuana use (path c). There is also no relationship present between neighborhood disorganization and parental and peer support (path a) which is also a requirement for mediation to be able to occur. Therefore, parental and peer support was not a mediator between neighborhood disorganization and substance use (alcohol, tobacco and marijuana). 


\section{Chapter V: Discussion}

\section{Introduction}

Few quantitative studies have explored the relationships of neighborhood disorganization, substance use, social support, depressive symptoms and functioning in adolescents involved in the juvenile justice system. This study examined these relationships in a group of adolescents participating in the Ohio Behavioral Health Juvenile Justice diversion program. This chapter will summarize the study findings, implications for nursing policy and practice, recommendations for future research and study limitations.

\section{Summary}

This study is a secondary data analysis that examined multiple risk and protective factors in a sample of adolescents involved in an Ohio juvenile justice diversion program. The purpose of this descriptive correlational study was to examine the relationships amongst neighborhood disorganization, social support and substance use (ever using alcohol, tobacco or marijuana) and functioning. This study also examined whether there were differences between neighborhood disorganization, social support and functioning based on personal characteristics (gender and race) and depressive symptoms. In addition to this, the differences between social support and functioning based on age of first substance use being before or age the age of 12 was explored. A path model was developed identifying the predictors of functioning controlling for age, gender and race. Lastly, the study examined whether social support mediated the relationship between neighborhood disorganization and functioning and neighborhood disorganization and ever using alcohol, tobacco or marijuana. 
Variables of interest for this study included: Neighborhood disorganization, social support (parental and peer), substance use (ever using alcohol, tobacco or marijuana), depressive symptoms and functioning. Neighborhood disorganization was measured using the U.S Census data at the zip code level including the following seven observed measures: percentage of female headed households with children under 18, percentage of residents with less than a high school education, percentage of renter occupied housing, percentage of residents who were employed, percentage of residents using supplemental security income, percentage receiving cash public assistance and the percentage of families below the poverty line. Social support was a self-report measure taken from the Youth Information Questionnaire (YIQ) that looked at the adolescent's perception of peer and parental social support. The Substance-Use Survey Revised provided information regarding whether the adolescent had ever used alcohol, tobacco and marijuana and also provided information related to the age of first substance use. Depressive symptoms was measured via the Trauma Symptom Checklist for Children (TSCC) and youth were classified as either "depressed" or "non-depressed" based on their standardized score. Functioning was measured by the Ohio Youth Problem, Functioning and Satisfaction Scale looking at the social workers report of the adolescent's functional capacity in a variety of daily activities including interpersonal relationships, recreation, self-direction and motivation. Statistical analyses included: Pearson product-moment correlation matrix, independent samples t-test, multiple regression analysis and hierarchical multiple regression.

Bronfenbrenner's Ecological Model was the theoretical framework that guided this study and described how nested contexts influence adolescent growth and 
development. This project explored each system testing the interrelationships between the microsystem (functioning, substance use), mesosystem (social support) and the exosystem (neighborhood disorganization). This theory explains the interconnectedness that exists between each system and explores how the interchange can impact growth and development. The study found parental social support to be the strongest predictor of functioning. Adolescence is recognized as a developmental stage where youth begin to define their identify and foster relationships with peer groups however this finding suggest that parents still have a vital role in their adolescent's pro-social development (Burke, Mulvey, Schubert \& Garbin, 2014). A positive parent-child relationship with secure bonding can potentially buffer the adverse effects that are often associated with juvenile delinquency and deviant behavior (Losel \& Farrington, 2012).

\section{Findings}

\section{Characteristics of Participants}

This study was a secondary data analysis of youth involved in the Behavioral Health Juvenile Justice (BHJJ) Initiative. The BHJJ services youth 10-18 years old with a DSM diagnosis and significant behavioral, cognitive or affective impairment. The subsample ( $\mathrm{n}=408)$ was comprised of participants who completed the Youth Information Questionnaire (YIQ) at intake between 2006-2007. All of the youth resided in the state of Ohio representing 7 counties and 96 zipcodes. The sample was primarily white and male with a mean age of 15 years old. The majority (79.9\%) of the youth lived with their biological parents and over half of the sample (53.2\%) was at risk for out of home placement. 


\section{Neighborhood Disorganization}

Neighborhood disorganization research suggests that there are social and economic characteristics of one's environment that can impact an adolescent's well-being (Browning, Soller \& Jackson, 2015). In addition to this, increased crime, violence, substance use and gang activity in a community can provide increased opportunity for an adolescent to engage in deviant behaviors (Leventhal \& Brooks-Gunn, 2000). This study examined the relationships between neighborhood disorganization, social support, and substance use (ever using alcohol, tobacco or marijuana) and found neighborhood disorganization to be negatively correlated with functioning. This suggests that adolescents who reported higher functioning lived in neighborhoods with less disorder. This finding is consistent with previous research that details that disadvantaged and unstable neighborhoods have fewer resources, job opportunities and less collective efficacy and social control when compared to more advantaged and stable neighborhoods with less disorganization (Wilson et al., 2005; Chen, Sheth, Elliott \& Yeager, 2004).

This study also examined whether there were differences in neighborhood disorganization based on gender and race. The study found that females reported higher levels of neighborhood disorganization than males. There is research suggesting that neighborhood characteristics have a different impact on delinquency in females compared to males. Disorganized neighborhoods are characterized by high residential instability, low socioeconomic status, segregation and isolation that result in weak social control (Sampson, 1997, Sampson \& Groves, 1989). This weak social control network allows criminal activity to go unnoticed by those who may not be immediately impacted. Females may also respond differently to these neighborhood conditions compared to 
males. Girls living in disorganized neighborhoods are exposed to more community violence, intra-familial violence, peer conflict and family dysfunction (Kroneman, Loeber \& Hipwell, 2004). Studies have consistently shown that problem behaviors in females are more prevalent in disadvantaged neighborhoods compared to more stable communities (Moffitt \& Caspi, 2001). Kellam, Ling, Merisca, Brown and Lalongo (1998) found that girls going to schools in neighborhoods with high disorganization were 10 times more likely to be highly aggressive when compared to girls residing in communities with less disorder.

In addition to gender differences, the study also found racial differences suggesting that non-whites resided in neighborhoods with higher disorganization than whites. These findings are consistent with current research related to racial differences in communities with high neighborhood disorganization. Historically, African Americans and Hispanics have experienced prejudice and discrimination in urban housing markets causing residential segregation to rise to unprecedented amounts in these communities (Massey, Gross \& Eggers, 1991). Cultures that experience rapid in-migration and slow socioeconomic mobility tend to display relatively higher levels of segregation while communities with rapid economic mobility and slower rates of in-migration tend to be more integrated. (Massey \& Hajnal, 1995). The measures that are used to define neighborhood disorganization can include poverty, crime, socioeconomic status, unemployment rates and educational level (Sampson, 1997, Sampson \& Groves, 1989). Racial minorities tend to be over-represented in these impoverished neighborhoods, residing in communities characterized by socioeconomic disadvantage, crime, violence and residential instability (De la Roca, Ellen O'Regan, 2014). 


\section{Substance Use}

Substance use among adolescents is a significant public health concern. Substance use increases an adolescent's risk for motor vehicle accidents, suicide, pregnancy and high-risk sexual behavior. Although many adolescents that experiment with these substances do not go on to develop a substance abuse problem (Fields \& Teitelbaum, 2012). Substance use has also been linked to social, economic and societal problems including juvenile delinquency, mental health problems and substance abuse in adulthood. In the study, there was a positive correlation between adolescents who reported ever-trying alcohol and ever-trying cigarettes and marijuana. There was also a positive correlation between ever-trying cigarettes and marijuana. These findings are consistent with research suggesting that adolescents who have used one substance are

more likely to have tried other substances (Fields \& Teitelbaum, 2012; Duncan, Strycker \& Duncan, 1999). Research suggests that if an adolescent uses tobacco or alcohol they are 65 times more likely to use marijuana than children who have never smoked or drank (Nkyi, 2015).

The neuropsychological effects of marijuana use have been well documented in research. Marijuana use has been associated with deficits in cognitive function as well as short-term and working memory deficits (Pardini et al., 2015; Volkow, Baler, Compton \& Weiss, 2014). It can also impact processing speed, attention, working memory and problem solving abilities (Jacobus, Bava, Cohen-Zion, Mahmood \& Tapert, 2008). This study found marijuana use to be negatively correlated with functioning. Adolescent marijuana use has been associated with a variety of school problems such as lower grades, truancy, and school drop-out (Pardini et al, 2015; Schweinsburg, Brown \& 
Tapert, 2008). A study by Fergusson \& Boden (2008) found statistically significant associations between marijuana use in adolescence and a range of adverse outcomes in later life. Increased marijuana use was associated with poorer educational outcomes, lower income, increased dependence on the welfare system, poor social relationships and decreased overall life satisfaction.

Research also suggests the younger a child begins to use tobacco, alcohol, marijauna or other drugs, the higher the risk for severe health problems and abuse carrying over into adulthood. Conditions such as low self-esteem, depression, aggression, delinquency and poor academic performance have all been linked to early substance use (Moss, Chen \& Yi, 2014; Dishion, Nelson \& Kavanagh, 2003). A study conducted by Sung, Erkovili, Angold \& Costello (2004) examined the effects of age of first use and a history of a psychiatric disorder on the development of substance use disorders by the age of 16 . The study found that among users without early conduct problems substance use before the age of 13 led to an $11 \%$ prevalence of substance use disorders by the age of 16. Similar to these findings, Moss, Chen \& Yi (2014) found the elevated risk of adult substance use disorders to be linked to multiple types of substances tried before the age of 16. Early regular use of cigarettes appeared to protect against alcohol use and early users of alcohol, marijuana and cigarettes had a lower prevalence of binge drinking. However, adolescents with early use of alcohol and marijuana with or without early use of cigarettes appeared to be at a significantly increased risk for both abuse and dependence in young adulthood. Even though there was no statistical significance between social support and functioning by age of first drug use in this study, the p-value 
of 0.06 suggests a trend toward lower peer support and functioning for youth who used drugs before the age of twelve.

\section{Social Support}

Although there are many forms of support, perceived support is the most relevant for well-being throughout one's lifetime (Barrera, 1986). Support serves as a protective factor by enhancing personal resources such as self-esteem, confidence and personal control (Turner \& Marino, 1994). The study found peer and parental support to be correlated to one another as well as functioning. This suggests that adolescents who identified themselves as having a supportive adult figures in their life also had a strong support network with their peers. In addition to this, the presence of peer and parental social support was positively correlated with functioning. Youth thrive in environments with supportive parents who promote pro-social behaviors and values (Reynolds \& Crea, 2015; Parker \& Benson, 2004). These youth understand what is expected of them and will gravitate to peer groups who share similar beliefs and values. Lack of strong positive relationships with parents increases one's opportunity to engage in deviant behaviors with peers (Reynolds \& Crea, 2015).

Social support can serve as a protective or risk factor with substance use. There is some evidence to suggest that parental and peer support can both be correlated to substance use and function but operates in opposite directions to one another. Peer and parental support networks may have different influences toward health-related behaviors suggesting that adolescent behaviors might be best predicted from the combination of their perception of parental and peer support. These findings are inconsistent with some research regarding cigarette use in adolescents. Social support is typically viewed as a 
positive influence on health and students with negative social support networks is often at risk to develop poor health behaviors (Wills, Resko, Ainette, Mendoza, 2004). Youth are less likely to smoke when their parents are involved and supportive. Several studies have also investigated the intergenerational influences of adolescent cigarette use finding it to be both heritable and influenced by environmental and social factors. In particular, adolescent's risk of smoking increased if they had a parent who smoked (Vuolo \& Staff, 2013). A study conducted by Vuolo \& Staff found that even in light smokers who reduced or quit in adulthood had children who were 3.5 times more likely to smoke than children of non-smokers. Although, this study did not capture evidence related to the parent's cigarette use, one possible explanation for our finding is that youth who reported higher parental social support may be modeling the behavior from their parents. The findings also suggest that adolescent substance use is a complex process that may involve several pathways for initiation that are not unidirectional or completely understood at this time.

Peer Support

Peer social support was positively correlated with both cigarette and alcohol use. Peer social support has been linked as a primary factor for adolescents to initiate cigarette smoking and reduce their attempts to quit smoking (Hall \& Valente, 2007; Von Ah, Ebert, Ngamvitroj, Park, \& Kang, 2005). Adolescents with friends who use substances are more likely to initiate smoking than those with friends who do not smoke (Vuolo \& Staff, 2013). A study by Wills, Resko, Ainette \& Mendora (2004) tested comparative effects of parental and peer support on adolescent substance use and found peer support to be positively related to substance use. Youth also tend to associate and socialize with 
peers who have similar interest thus increasing the risks of adolescents to adopt maladaptive behaviors and traits from their social groups. Adolescence is a developmental period where youth have a strong interest in social comparison, acceptance and reward and may partake in unacceptable behaviors in order to be accepted. Youth may also adopt attitudes and behaviors of peers that they may want to associate with regardless of whether or not the behavior is viewed as socially acceptable.

\section{Parental Support}

This study also found differences in parental social support based on gender and race. Males reported higher levels of parental social support than females and whites reported more parental social support than non-whites. Variations in the levels of support based on personal characteristics such as gender and race are largely influenced by a collection of factors versus one signal pathway (Evans-Chase, 2014; Espinosa, Sorensen \& Lopez, 2013). The availability and perception of social support is highly influenced by cultural norms and can vary across various populations. Females as well as youth of color who are involved in the juvenile justice system come from complex social environments often disproportionately exposed to violence, trauma and adverse childhood events (Evans-Chase, 2014; Espinosa, Sorensen \& Lopez, 2013; Vaux, 1985). Youth of color are also exposed to a range of risk factors within their communities that includes coming from single parent homes. Teenagers that are left unsupervised while their parent is working may be more inclined to engage in more defiant behaviors outside of the home due to the lack of supervision and parental monitoring. Each of these factors can directly impact the adolescent's ability to develop positive pro-social relationships with their peers. 
Peer and parental social support was hypothesized to serve as a mediator between neighborhood disorganization and functioning and neighborhood disorganization and substance use. A hierarchical regression model was used to test this potential mediating relationship. There are no previous research studies that have examined the mediating effect of peer and parental social support between neighborhood disorganization and functioning and neighborhood disorganization and substance use in juvenile justice involved youth. Although, previous research studying similar concepts has demonstrated that social support functioned as a mediator our results were not consistent with those finding (Nilsen, Karevold, Roysamb, Gustavson \& Mathiesen, 2013).

\section{Depressive Symptoms}

Adolescents are exposed to several challenges and stressors that can jeopardize their capacity to regulate affect and successfully manage relationships with peers (Caprara, Gerbino, Paciello, Di Giunta \& Pastorelli, 2010). Academic pressure, gaining independence from parents, relationships with peers and physical maturation are accompanied by a drastic increase in the frequency at which stressful life events can occur. Chronic exposure to stressors and trauma is associated with psychological stress and physiological consequences that may elevate to maladaptive behaviors and problems, such as depression (Frison \& Eggermont, 2014; Brenner, Zimmerman, Bauermeister \& Caldwell, 2013). Depression occurs more frequently with this age group, particularly after the onset of puberty (Cocozza, \& Skowyra, 2000). Age was found to be a predictor of depressive symptoms, which is consistent with research findings related to the presentation of depressive symptoms in this population. 
When comparing depressed to non-depressed youth, this study found depressed youth to reside in neighborhoods with less disorganization. These findings are not consistent with other research that has found an association between residing in disorganized neighborhoods and poorer mental health outcomes (Kim, 2010). Neighborhood disorganization may undermine the buffering effect of family and peer social support through a stress pathway. Adolescents living in disadvantaged neighborhoods encounter more frequent and sustained stressful events that can potentially last over the course of several years (Brenner, Zimmerman, Bauermeister \& Caldwell, 2013). Some research suggest that children exposed to continued violence may experience a desensitization process such that stressors have little impact on their overall well-being (Wright \& Fagan, 2013, Steward, Simons \& Conger, 2002; Farrell \& Bruce, 1996). Therefore, youth reporting more depressive symptoms in the sample may attribute their symptomology to reasons other than the conditions of the neighborhoods in which they reside. Mental illness is a complex health condition that often results from a combination of several risk factors in comparison to one clearly defined pathway (Frison \& Eggermont, 2014: Cocozza, \& Skowyra, 2000).

Researchers have documented positive associations between social support and psychological well-being among youth and inverse associations between social support and depression (Hussong 2000). Social support plays a critical role in health, life satisfaction and positive adjustment (Cheng et al, 2014). Contact with individuals who share a close, affectionate bond is associated with feelings of belonging, pleasure and reduced anxiety (Newman, Newman, Griffin, O'Connor \& Spas, 2007). In this study, youth with depressive symptoms reported having less peer social support than those who 
were not depressed. They also reported lower functioning compared to non-depressed youth. This is consistent with research findings suggesting that adolescents who were less socially connected were also less likely to receive emotional and physical support in times of stress that also had a direct impact on their daily functioning (Hussong, 2000). Similar to these findings, a study by Newman, Newman, Griffen, O’Connor \& Spas (2007) found the transition to high school to be accompanied by a decline in a sense of belonging and an increase in depressive symptoms. Cheng et al (2014) found social support from family and neighborhood resources to be positively associated with an adolescents' hope score universally, suggesting that the presence of social support in an adolescent's life is an important resilience factor from which to build.

\section{Implications for Nursing Practice}

Judicial processing provides a critical opportunity to intervene in an adolescents life and potentially prevent the long-term adverse outcomes of delinquency (Skowyra \& Cocozza, 2007). The conditions associated with detention such as separation from loved ones, solitary confinement and overcrowded facilities may increase one's risk for mental distress and suicidality (Gallagher \& Dobrin, 2006). Nurses are ideal providers to identify and advocate for youth who may benefit from alternative sentencing options such as diversion. Nurses can serve an integral role on a multi-disciplinary team providing health education and performing comprehensive assessments for youth involved in the juvenile justice system. This population is more likely to have a mental health diagnosis, engage in drug, alcohol or tobacco use and also have a higher incidence of suicide. Research has documented that 70-90 percent of youth in the juvenile justice system meet criteria for atleast one psychiatric disorder (Assink et al, 2015). This population is also at increased 
risk for various medical conditions that may require ongoing follow up care. A study by Feinstain, Lampkin, Lorish, Kleiman \& Masiak (1998) found that 1/5 of the juvenile delinquents reported a history of hospitalization for a medical or surgical reason. The majority of hospitalizations were from trauma, fights, gunshot wounds or an unrelated athletic activity. Only $33.6 \%$ of the respondents reported having an established medical provider. This is particularly alarming in a population of youth at risk for substance use, sexually transmitted diseases and pregnancy. National surveys have also consistently found higher rates of traumatic injury, violence exposure, tuberculosis and dental problems amongst this population. Therefore, identifying these adolescents who may be at risk early and providing them with interventions that are tailored to their individualized needs is vital in order to reduce adverse health outcomes in adulthood. Assessment and treatment must incorporate developmentally, culturally and contextually appropriate interventions (Penney \& Moretti, 2005). Effectively identifying and treating psychiatric disorders not only serves the adolescent but also the communities to which they will return.

Similar to previous research, parental social support was identified as a predictor of functioning. The parent-child relationship, whether positive or negative is a significant factor in adolescent development (Cutrona, 2000). Development of close relationships with parents and other adult role models provides encouragement, support and clear expectations regarding one's behavior (Asscher, Wissink, Dekovic, Prinzie \& Stams, 2014). In general, youth who identify themselves as having an adult figure they can rely on are able to more effectively manage their behaviors and possess the skills and opportunities to be successful. A recent survey by the Centers for Juvenile Justice reform 
(2008) identified family involvement as one of the most important and challenging issues to address in juvenile justice reform. Effective programs address the needs of the family while also ensuring familial stability and financial resources are available (Washburn et al, 2015). Nurses can continue to help facilitate and encourage the involvement of the family unit while also advocating for the needs of the adolescent.

\section{Study Limitations}

The following limitations need to be considered when one interprets the study findings. The Behavioral Health Juvenile Justice Initiative is a convenience sample of Ohio adolescents referred to the juvenile justice program. Although there is some diversity present related to race, gender and age, the sample was comprised entirely of Ohio youth residing mostly in urban and rural areas. Youth who met the eligibility criteria were recommended for enrollment and there is no control group available for comparison. Therefore, due to the convenience sample, generalizability of the findings and comparison to other populations should be interpreted with caution. These results are consistent with other studies that have examined similar concepts in this population and add to the growing literature related to juvenile diversion programs specifically those that target youth with behavioral health concerns.

Another limitation is related to the fact that this study was a secondary data analysis focusing specifically on data collected at intake in 2006-2007. A major limitation of a secondary analysis is that the researcher did not participate in the data collection process and is limited to what variables they are able to use and the way in which certain phenomena can be analyzed. Since the researcher did not participate in the collection process they had to make inferences that appropriate protocol and procedures 
were followed. The researcher also utilized documentation from the original study, published finding and consultation with the primary investigator and research team to address any methodological concerns they may have encountered during data collection.

Adolescents particularly those involved in the juvenile justice system represent a vulnerable population in research. Therefore, it is important that the researcher is aware of the studies IRB process in addition to obtaining information regarding how parental consent and adolescent assent was obtained for the project. Participants should be well informed, know the objectives of the study in addition to any foreseeable risks associated with participation. Providing assent ensures that the participant does not feel coerced or forced to participate in the research study. Adolescents who are being diverted from formal court processing must understand that their participation in a research study is completely independent of their court involvement and participation will not impact the course of their sentencing or programming.

The third limitation is related to measurement scales and the way in which social support was measured. Since the YIQ was only administered in 2006-2007 using this measure as a means to examine social support significantly limited the sample size and did not allow analysis of the youth who enrolled in the study after the questionnaire was omitted from the study packet. The questionnaire asks the youth six questions related to their perceptions of whether or not they have an adult or friend in their life that they can talk to, ask for help or have fun with. Although this questionnaire demonstrated acceptable reliability and internal consistency, using a standardized scale that has been tested in similar populations would have been more ideal. 
An additional limitation related to a measurement tool is associated with the way that substance use was measured for this study. Ideally, one would want to gather information describing substance use behaviors and trends particularly related to frequency and duration of one's substance use. The researcher originally wanted to examine the use of alcohol, tobacco and marijuana over the course of the last 30 days but was unable to continue with this variable for analysis due to the considerable amount of missing data that was present in the subsample. There were also concerns related to the adolescent's recall, accuracy and willingness to provide an honest report to a sensitive question.

The fourth limitation is related to the way in which neighborhood disorganization was measured. Currently there is no clear consensus in the literature related to whether objective (U.S Census data) or perceptual (neighborhood response) is the most accurate means to capture this phenomenon. Objective measures provide insight into the structural characteristics of the community while perceptual measures detail the social cohesion and structural conflicts that may exist in one community compared to another (Hart \& Waller, 2013; Hayes, 2007). Census block, block groups and tracts are the most common boundaries used as neighborhood proxies in neighborhood disorganization research (Sperling, 2012). Operationally defining "neighborhoods" in terms of administrative boundaries makes sense especially since indicators of social disorganization that are used to explain neighborhood effects on crime are contained in census data and can easily be obtained. However, doing so does not take into account that communities are socially constructed and may vary from one street to another depending on the views and perspectives of those who reside there. Compared to census blocks, zip codes cover a 
larger geographic area and are designed to meet the needs of the U.S postal delivery service and are subject to change at any time. Zip codes do not have a maximum and minimum population threshold and often cover wide demographic and socioeconomic characteristics.

The last limitation is related to using the 2010 U.S Census data to obtain values for each of the observed neighborhood characteristics. U.S Census data is supported and funded by the U.S government and collects a broad range of information to help one understand community traits and trends (Sperling, 2012; Hayes, 2007). Although informative and useful, Census data does have its limitations. U.S Census data does not provide individual level information and though it can help one to understand how two places differ from one another it does not allow one to study the individual influences of one household compared to another. Since data is only gathered on a portion of households rather than the full population there are risks for sampling errors being introduced. In addition to this, if a person changes residences during the data collection period they could possibly receive and return a census form for a household that's already been accounted for in the data. Lastly, this form of data collection excludes high-risk youth who may be homeless, living in shelters, residing with family or in the foster care system.

\section{Recommendations for Future Research}

Juvenile diversion redirects youth from formal processing in the juvenile justice system into intensive treatment and therapeutic programming. Diversion programs are cost-effective; reduce the burden on the court system and free-up limited resources and services for more high risks offenders. Diversion is designed to reduce the stigma and 
negative consequences that youth may experience if they are labeled a delinquent (Becker, 1963). It also is intended to reduce the negative impacts of formal system processing. Research suggests that reoffending increases as youth are further processed in the juvenile justice system (Guckenburg, 2010). Although diversion programs have illustrated its benefits further research should address specific types of treatment modalities that are most effective. The Behavioral Health Juvenile Justice (BHJJ) has demonstrated promising results for Ohio youth in the juvenile justice system however there isn't much consistency related to the type of programs that are offered from one county to the next. Although, there is consensus that intensive family treatment works best for these youth future research should explore which modality is most advantageous and how to better address the mental health concerns that often presents in this population.

Parent involvement is widely recognized as a key factor for promoting positive child outcomes in education, mental health and juvenile justice (Burke, Mulvey, Schubert \& Garbin, 2014). The inclusion of parents has improved the effectiveness of treatment for youth, particularly those with mental health needs (Cohen \& Mannarino, 2000). Future research should continue to focus on exploring ways to better address the family as a unit instead of tailoring interventions to only the youth. Arising from a system of care approach, "family driven care" empowers parents and guardians to have a central role in the treatment goals and objectives of their children's care (Welsh \& Greenwood, 2015) This approach encourages collaboration between service providers and the parents are viewed as the active and equal participant in the treatment. Focus groups have revealed that parents often feel a sense of guilt and blame as a result of their adolescent's behavior 
(Luckenbill \& Yeager, 2009). Exploring ways to better address the family unit and empower the guardian will assist them to better support their adolescents progress throughout the program and decrease the rate of recidivism.

\section{Conclusion}

This descriptive, correlational secondary analysis of the Behavioral Health Juvenile Justice Initiative examined the relationships of neighborhood disorganization, social support, substance use and functioning in a group of adolescents. The study also assessed the impact of depressive symptoms on overall functioning in these youth. The finding support previous research and contributes to the growing body of research related to juvenile offenders particularly those in diversion programs. Juvenile diversion programs are an effective alternative to traditional judicial court processing and helps to address some of the complex issues these youth face. Strengths of this study include the use of comprehensive, psychometrically sound instruments to measure complex phenomena amongst a high-risk population. The study also followed Bronfenbrenner's Social-Ecological Model, which is an established theoretical framework to guide the research design. This study contributes to the field of nursing research and examines concepts and social justice concerns that are not traditional explored in this field. Delinquency occurs in a social context influenced by communities, schools and families. Although the justice system is intended to be just and neutral there are instances when racial and gender biases occur. African American and Hispanic males have the greatest odds to be processed in the adult criminal court than non-Hispanic white youth even after adjusting for felony-level violent crimes (Washburn et al., 2008). Nurses serve a vital role as a patient advocate to ensure that appropriate programs and interventions are being 
offered for all youth involved in the juvenile justice system regardless of race or gender. Utilizing the nurse or advanced practice provider as a means to identify these at-risks youth in order to provide early individualized treatment options is critical to address their complex health needs. Researchers should continue to explore ways to improve the short and long-term behavioral health outcomes for these youth in addition to focusing on interventions that continue to foster, support and develop health relationships amongst families. 


\section{REFERENCES}

Abram, K. M., Choe, J. Y., Washburn, J. J., Romero, E. G., Teplin, L. A., \& Bassett, E. D. (2013). Functional impairment in delinquent youth. Juvenile Justice Bulletin, $1-12$.

Abrams, L. S. (2013). Juvenile justice at a crossroads: Science, evidence, and twentyfirst century reform. Social Service Review, 87(4), 725-752.

Ackard, D. M., Neumark-Sztainer, D., Story, M., \& Perry, C. (2006). Parent-child connectedness and behavioral and emotional health among adolescents. American Journal of Preventive Medicine, 30, 59-66.

Allen, J. P., Chango, J., Szwedo, D., Schad, M., \& Marston, E. (2012). Predictors of susceptibility to peer influence regarding substance use in adolescence. Child Development, 83(1), 337-350.

Anthony, J., \& Petronis, K. (1995). Early-onset drug use and risk of later drug problems. Drug and Alcohol Dependence, 40, 9-15.

Armstrong, T. D., \& Costello, E. J. (2002). Community studies on adolescent substance use, abuse, or dependence and psychiatric comorbidity. Journal of Consulting and Clinical Psychology, 70(6), 1224-1239.

Arthur, M.W, Briney, J.S, Hawkins, R.D, Abbott, B.L., Brooke-Weiss, R.F., Catalano. (2007). Measuring risk and protection in communities using the communities that care youth survey. Evaluation and Program Planning, 30 (2):197-211.

Asarnow, J. R., Zeledon, L. R., D'Amico, E., LaBorde, A., Anderson, M., Avina, C., ... Arslanian, T. (2014). Depression and health risk behaviors: Towards optimizing primary care services strategies for addressing risk. Primary Health Care, 4(1), $1-15$.

Asscher, J. J., Wissink, I. B., Dekovic, M., Prinzie, P., \& Stams, G. (2014). Delinquent behavior, poor relationship quality with parents, and involvement with deviant peers in delinquent and non-delinquent adolescents: Different processes, informant bias or both? International Journal of Offender Therapy and Comparative Criminology, 58(9), 1001-1019.

Assink, M., Van der Put, C. E., Hoeve, M., De Vries, S., Stams, G., \& Oort, F. (2015). Risk factors for persistent delinquent behaviors among juveniles: A metaanalytic review. Clinical Psychology Review, 42, 47-61.

Bachman, J., Patrick, J., O'Malley, M., \& Freedman-Doan, P. (2013). Adolescent work intensity, school performance, and substance use: Links vary by race/ethnicity and socio-economic status. Developmental Psychology, 49(11), 1-16. 
Barnert, E. S., Perry, R., Azzi, V. F., Shetgiri, R., Ryan, G. W., Dudovitz, R. N., . . Zima, B. T. (2015). Incarcerated youth perspectives on protective factors and risk factors for juvenile offending: a qualitative analysis. American journal of public health, 105(7), 1365-1371.

Baron, R. M., \& Kenny, D. A. (1986). The moderator-mediator variable distinction in social psychological research: Conceptual, strategic, and statistical considerations. Journal of Personality and Social Psychology, 51, 1173-1182.

Barrera, M (1986). "Distinctions between social support concepts, measures, and models". American Journal of Community Psychology 14 (4): 413-445.

Beaver, K. M., Connolly, E. J., Schwartz, J. A., Al-Ghamdi, M. S., \& Kobeisy, A. N. (2013). Genetic and environmental contributions to stability and change in levels of self-control. Journal of Criminal Justice, 41(5), 300-308.

Beaver, K. M., DeLisi, M., Mears, D. P., \& Stewart, E. (2009). Low self-control and contact with the criminal justice system in a nationally representative sample of males. Justice Quarterly, 26, 695-715.

Beaver, K. M., DeLisi, M., Vaughn, M. G., \& Wright, J. P. (2010). The intersection of genes and neuropsychological deficits in the prediction of adolescent delinquency and low self-control. International Journal of Offender Therapy and Comparative Criminology, 54, 22-42.

Bechtold, J., Hipwell, A., Lewis, D. A., Loeber, R., \& Pardini, D. (2016). Concurrent and sustained cumulative effects of adolescent marijuana use on subclinical psychotic symptoms. The American Journal of Psychiatry, 173(8), 781-789.

Bender, D., \& Losel, F. (2011). Bullying at school as a predictor of delinquency, violence and other anti-social behavior in adulthood. Criminal behaviour and Mental Health, 21, 99-106.

Bergseth, K. J., \& Bouffard, J. A. (2012). Examining the effectiveness of a restorative justice program for various types of juvenile offenders. International Journal of Offender Therapy and Comparative Criminology, 1-22.

Bhatta MP, Jefferis E, Kavadas A, Alemagno SA, Shaffer-King P (2014) Suicidal Behaviors among Adolescents in Juvenile Detention: Role of Adverse Life Experiences. PLoS ONE 9(2): e89408

Blakemore, S., Burnett, S., \& Dahl, R. (2010). The role of puberty in the developing adolescent brain. Human Brain Mapping, 31, 926-933.

Blakemore, S.J., Robbins, T.W., 2012. Decision-making in the adolescent brain. Nature 
Neuroscience 15, 1184-1191.

Bloom, D.E., Cafiero, E.T., Jané-Llopis, E., Abrahams-Gessel, S., Bloom, L.R., Fathima, S., Feigl, A.B., Gaziano, T., Mowafi, M., Pandya, A., Prettner, K., Rosenberg, L., Seligman, B., Stein, A.Z., \& Weinstein, C. (2011). The Global Economic Burden of Noncommunicable Diseases. Geneva: World Economic Forum.

Bradshaw, C. P., Schaeffer, C. M., Petras, H., \& Ialongo, N. (2010). Predicting negative life outcomes from early aggressive-disruptive behavior across trajectories: Gender differences in maladaptation across life domains. Journal of Youth and Adolescence, 39, 953-966.

Briere, J. (1996). Trauma Symptom Checklist for Children: Professional Manual. Odessa, FL: Psychological Assessment Resources, Inc.

Briggs, D. C. (2008). Comments on Slavin: Synthesizing causal inferences. Educational Researcher, 37, 15-22.

Bright, C. L., Hergenroeder, N. S., \& Morris-Compton, D. (2014). Diversion from the juvenile justice system: Observations of a teen court program. Journal of Community Practice,22(3), 385-401.

Bronfenbrenner, U. (1979). The ecology of human development: Experiments by design and nature. Cambridge, MA: Harvard University Press.

Bronfenbrenner, U. (2005). Making human beings human: Bioecological perspectives on human development. Thousand Oaks, CA: Sage.

Brook, D. W., Brook, J. S., Zhang, C., Cohen, P., \& Whiteman, M. (2002). Drug use and the risk of major depressive disorder, alcohol dependence, and substance use disorders.Archives of General Psychiatry, 59(11), 1039-1044.

Browning, C. R., Soller, B., \& Jackson, A. L. (2015). Neighborhoods and adolescent health-risk behavior: An ecological network approach. Social Science and Medicine, 125, 163-172.

Bruinsma, G., Pauwels, L., Weerman, F., \& Bernasco, W. (2013). Social disorganization, social capital, collective efficacy and the spatial distribution of crime and offenders. British Journal of Criminology, 53, 942-963

Burke, J. D., \& Loeber, R. (2015). The effectiveness of the Stop Now and Plan (SNAP) Program for boys at risk for violence and delinquency. Prevention Science, 16, 242-253.

Burrell, T., Cahalan, C., Cimaglio, B., Dennis, M. L., Head-Dunham, R., Henggeler, S. 
W., ... Levy, S. (2014). Principles of Adolescent Substance Use Disorder Treatment: A Research-Based Guide. Retrieved from https://www.drugabuse.gov/publications/principles-adolescent-substance-usedisorder-treatment-research-based-guide/acknowledgements

Byrnes, H. F., Miller, B. A., Chamratrithirong, A., Rhucharoenpornpanich, O., Cupp, P. K., Atwood, K. A., Fongkaew, W et al. (2011). The roles of perceived neighborhood disorganization, social cohesion, and social control in urban Thai adolescents substance use and delinquency. Youth \& Society, 45(3), 404-427.

Cahill L. (2006): Why sex matters for neuroscience. Nature Reviews Neuroscience, 7 , 477-484.

Callahan, L., Cocozza, J., Steadman, H., \& Tillman, S. (2012). A national survey of the U.S juvenile mental health courts. Psychiatric Services, 63(2), 130-134.

Campaign for Tobacco-Free Kids. Smoking's immediate effects on the body. http://tobaccofreekids.org/research/factsheets/pdf/0264.pdf

Campbell, D.T., \& Stanley, J.C. (1963). Experimental and quasi-experimental designs for research. Boston: Houghton Mifflin.

Caprara, G. V., Gerbino, M., Paciello, M., Di Giunta, L., \& Pastorelli, C. (2010). Counteracting depression and delinquency in late adolescence. European Psychologists, 15, 34-48.

Catalano RF, Hawkins JD. The social development model: a theory of antisocial behavior. In: HawkinsJD, ed. Delinquency and Crime: Current Theories. New York, NY: Cambridge University Press; 1996: 149-197.

Caughy M.O., Nettles S.M, O'Campo P.J. (2008) The effect of residential neighborhood on child behavior problems in first grade. American Journal of Community Psychology. 42(1-2):39-50.

Chapman, J. F., Desai, R. A., \& Falzer, P. R. (2006). Mental health service provision in juvenile justice facilities: Pre- and postrelease psychiatric care. Child and Adolescent Psychiatric Clinics of North America, 15(2), 445-458.

Chandra, A.; Minkovitz, C. S. 2006. Stigma Starts Early: Gender Differences in Teen Willingness to Use Mental Health Services. Journal of Adolescent Health 38: $754 \mathrm{e} 1-754 \mathrm{e} 8$.

Chein, J., Albert, D., O'Brien, L., Uckert, K., \& Steinberg, L. (2010). Peers increase adolescent risk taking by enhancing activity in the brain's reward circuitry. 
Developmental Science,14(2), F1-F10.

Chen, K., Sheth, A. J., Elliott, D. K., \& Yeager, A. (2004). Prevalence and correlates of past-year substance use, abuse, and dependence in a suburban community sample of high school students. Addictive Behaviors, 29(2), 413-423.

Children's Defense Fund, Juvenile Detention Reform in Ohio- Fact Sheet (2010), available at http://www.childrenslawky.org/wpcontent/uploads/2012/07/rethinking-juvenile-detention-fact- sheet.pdf.

Choudhurry, S. (2010). Culturing the adolescent brain: What can neuroscience learn from anthropology? Social Cognitive and Affective Neuroscience, 5, 159-167.

Cicchetti, D. (2010). Resilience under conditions of extreme stress: A multilevel perspective. World Psychiatry, 9, 145-154.

Cleveland, M., Feinberg, M., Bontempo, D., \& Greenberg, M. (2008). The role of risk and protective factors in substance use across adolescence. Journal of Adolescent Health, 43, 157-164.

Cocozza, J., \& Skowyra, K. (2000). Youth with mental health disorders: Issues and emerging responses. Juvenile Justice, 7, 3-13.

Coffey, C., Vert, K., Wolfe, R., Cini, E., \& Pattson, G. C. (2003). Mortality in young offenders: Retrospective cohort study. British Medical Journal, 326(1064), 1-4.

Cohen, J. A., \& Mannarino, A. P. (2000). Predictors of treatment outcome in sexually abused children. Child Abuse \& Neglect, 24(7), 983-994.

Cohen, M. A., \& Piquero, A. R. (2009). New evidence on the monetary value of saving a high risk youth. Journal of Quantitative Criminology, 25, 25-49.

Colwell, B., Villarreal, S. F., \& Espinosa, E. M. (2012). Preliminary outcomes of preadjudication diversion initiative for juvenile justice involved youth with mental health needs in Texas. Criminal Justice and Behavior, 39(4), 447-460.

Cook, T. D., \& Campbell, D. T. (1979). Quasi-experimentation: Design \& analysis issues for field settings. Boston: Houghton Mifflin.

Corty, E. W. (2007). Using and interpreting statistics: A practical text for the health, behavioral, and social sciences. St. Louis, MO: Mosby.

Costello, E. J., He, J. P., Sampson, N. A., Kessler, R. C., \& Merikangas, K. R. (2013). Services for adolescents with psychiatric disorders: 12-month data from the National Comorbidity Survey-Adolescent. Psychiatric Services, 65(3), 459. 
Cox, R. B., Burr, B., Blow, A. J., \& Parra Cardona, J. R. (2011). Latino adolescent substance use in the United States: Using the Bioecodevelopmental model as an organizing framework for research and practice. Journal of Family Theory \& Review, 96-123

Crockenberg, S.C., Leerkes, E. M., \& Barrig Jo, P.S. (2008). Predicting aggressive behavior in the third year from infant reactivity and regulation as moderated by maternal behavior. Development and Psychopathology, 20, 37-54.

Crone, E., \& Dahl, R. (2012). Understanding adolescence as a period of social-affective engagement and goal flexibility. Nature Reviews Neuroscience, 13, 636-650.

Cronk, C. E., \& Sarvela, P. D. (1997). Alcohol, tobacco, and other drug use among rural/small town and urban youth: A secondary analysis of the monitoring the future data set. American Journal of Public Health, 87(5), 760-764.

Cuellar, A. E., McReynolds, L. S., \& Wasserman, G. A. (2005). A cure for crime: Can mental health treatment diversion reduce crime among youth? Journal of Policy Analysis and Management, 25(1), 197-214.

Cutrona, C.E (2000) Social support principles for strengthening families: Messages from America. In: Family Support: Direction from Diversity. Jessica Kingsley Publishing, London.

De la Roca, J., Ellen, I., \& O'Regan, K. (2014). Race and neighborhoods in the 21st century. What does segregation mean today? Regional Science and Urban Economics, 47, 138-151.

DeLisi, M., \& Vaughn, M. G. (2014). Foundation for a temperament-based theory of antisocial behavior and criminal justice system involvement. Journal of Criminal Justice, 42, 10-25.

DeLisi, M., Angton, A., Behnken, M. P., \& Kusow, A. M. (2013). Do adolescent drug users fare the worst? Onset type, juvenile delinquency, and criminal careers. International Journal of Offender Therapy and Comparative Criminology, 1-16.

Dembo, R., Briones, R., Gulledge, L., Koras, L., Winters, K. C., Beienko, S., \& Greenbaum, P. E. (2012). Stress, mental health, and substance abuse problems in a sample of diversion program youth: An exploratory latent class analysis. Journal of Child and Adolescent Substance Use, 19(34), 130-155.

Dever, B. V., Schulenberg, J. E., Dworkin, J. B., O'Malley, P. M., Kloska, D. D., \& Bachman, J. G. (2012). Predicting risk-taking with and without substance use: The effects of parental monitoring, school bonding, and sports participation. Prevention Science, 13, 605-615. 
De Vries, S. L., Hoeve, M., Assink, M., Stams, G. J., \& Asscher, J. J. (2015). Practitioner review: Effective ingredients of prevention programs for youth at risk of persistent juvenile delinquency- recommendations for clinical practice. Journal of Child Psychology and Psychiatry, 56(2), 108-121.

Depression (PDF). National Institute of Mental Health (NIMH). Retrieved 12 February 2015.

E Y Deykin, J C Levy, and V Wells. (1987). “Adolescent depression, alcohol and drug abuse. American Journal of Public Health 77, (2), 178-182.

Dierkhising, C. B., Ko, S. J., Woods-Jaeger, B., Briggs, E. C., Lee, R., \& Pynoos, R. S. (2013). Trauma histories among justice-involved youth: Findings from the National Child Traumatic Stress Network. European Journal of Psychotraumatology, 4, 1-12.

Di Forti, M., Morrison, P. D., Butt, A., \& Murray, R. M. (2007). Cannabis use and psychiatric and cognitive disorders: the chicken or the egg? Current Opinion in Psychiatry, 20, 228-234.

Dishion, T. J., Nelson, S. E., \& Kavanagh, K. (2003). The Family Check-Up with highrisk young adolescents: Preventing early-onset substance use by parent monitoring. Behavior Therapy, 34, 553-571.

Dowell, K. A. \& Ogles, B. M. (2008). The Ohio Scales Youth Form: Expansion and validation of a self-report outcome measure for young children. Journal of Child \& Family Studies, 17(3), 291-305.

Drake, R. E., Mueser, K. T., Brunette, M. F., \& McHugo, G. J. (2004). A review of treatments for people with severe mental illnesses and co-occurring substance use disorders. Psychiatric Rehabilitation Journal, 27, 360-374.

Drewnowski A, Rehm CD, Solet D (2007). Disparities in obesity rates: analysis by ZIP code area. Social Science and Medicine. 65(12): 2458-63.

Dulin-Keita, A., Casazza, K., Fernandez, J., Goran, M., \& Gower, B. (2012). Do neighborhoods matter? Neighborhood disorder and long-term trends in serum cortisol levels. Journal of epidemiology and community health, 66(1), 24-29.

Duncan, S. C., Strycker, L. A., \& Duncan, T. E. (1999). Exploring associations in developmental trends of adolescent substance use and risky sexual behavior in high-risk population. Journal of Behavioral Medicine, 22(1), 21-34.

Duncan S. C., Alpert A., Duncan T.E., Hops H. (1997). Adolescent alcohol use development and young adult outcomes. Drug Alcohol Dependence, 49:39-48. 
Eberhardt MS, Ingram DD, Makuc DM, et al. Urban and Rural Health Chartbook. Health, United States, 2001. Hyattsville, Maryland: National Center for Health Statistics. 2001.

Elliott, D. S. (1998). Blueprints for violence prevention (Series Ed.). University of Colorado, Center for the Study and Prevention of Violence. Boulder, CO: Blueprints Publications.

Elliot, M. C., Leventhal, T., Shuey, E. A., Lynch, A. D., \& Coley, R. L. (2014). The home and the "hood': Associations between housing and neighborhood contexts and adolescent functioning. Journal of Research on Adolescence, 1-13.

Elliott, D. S., Wilson, W. J., Huizinga, D., Sampson, R. J., Elliott, a., \& Rankin, B. (1996). The effects of neighborhood disadvantage on adolescent development. Journal of Research in Crime and Delinquency, 33, 389-426.

Ellis, B. J., Dishion, T. J., Gray, P., Hawley, P. H., Volk, A. A., \& Figeredo, A. J. (2012). The evolutionary basis of risky adolescent behavior: Implications for science, policy and practice. Developmental Psychology, 48(3), 598-623.

Erin M. Espinosa, Jon R. Sorensen, and Molly A. Lopez. (2013) "Youth Pathways to Placement: The Influence of Gender, Mental Health Need and Trauma on Confinement in the Juvenile Justice System.” Journal of Youth and Adolescence 42,1824-36.

Espinosa, E. M., Sorensen, J. R., \& Lopez, M. A. (2013). Youth pathways to placement: The Influence of gender, mental health need and trauma on confinement in the juvenile justice system. Empirical Research, 42(12), 18241836.

Evans, S. Z., Simons, L. G., \& Simons, R. L. (2014). Factors that influence trajectories of delinquency throughout adolescence. Journal of Youth and Adolescence.

Evans-Chase, M. (2014). Addressing trauma and psychosocial development in juvenile justice-involved youth: A synthesis of the developmental neuroscience, juvenile justice and trauma literature. Laws, 3, 744-758.

Fagan, J. (2008). Juvenile crime and criminal justice: Resolving border disputes. The Future of Children, 18(2), 81-118.

Fagan, A. A., \& Wright, E. M. (2012). The effects of neighborhood context on youth violence and delinquency: Does gender matter? Youth Violence and Juvenile Justice, 10(1), 64-82.

Fagan, A. A., Wright, E. M., \& Pinchevsky, G. M. (2015). A multi-level analysis of the 
impact of neighborhood structural and social factors on adolescent substance use. Drug and Alcohol Dependence, 153, 180-186.

Farrington, D. P. (2012). Should the juvenile justice system be involved in early intervention.American Society of Criminology, 11(2), 265-273.

Fazel S, Benning R, Danesh J (2003) Suicide in male prisoners in England and Wales, 1978-2003. Lancet 366: 1301-1302.

Federal Bureau of Investigation (FBI). (2012). Crime in the United States, 2011. Washington, DC: U.S. Government Printing Office.

Feinstain RA, Lampkin A, Lorish CD, Klerman LV, Masiak R. (1998). Medical status of adolescents at time of admission to a juvenile detention center. Journal of Adolescent Health. 1998; 22:190-196.

Feinstein, E., Richter, L., \& Foster, S. (2012). Addressing the critical health problem of adolescent substance use through healthcare, research, and public policy. Journal of Adolescent Health, 50, 431-436.

Ferguson, L. (2004). External validity, generalizability and knowledge utilization. Journal of Nursing Scholarship, 36(1), 16-22.

Field, A. (2009). Discovering statistics using SPSS (3rd ed.). London: Sage.

Fields, Douglas (2008). "White Matter". Scientific American 298 (3): 54-61.

Fields J.B, \& Teitelbaum, S.A (2012). The Physician's Approach to Substance Use in Adolescents Northeast Florida Medicine, 63, 1, 22- 27

Fosco, G. M., Stormshak, E. A., Dishion, T. J., \& Winter, C. E. (2012). Family relationships and parental monitoring during middle school as predictors of early adolescent problem behavior. Journal of Clinical Child and Adolescent Psychology, 41(2), 202-213.

Fox, B. H., Perez, N., Cass, E., Baglivio, M. T., \& Epps, N. (2014). Trauma changes everything: Examining the relationship between adverse childhood experiences and serious, violent crimes and chronic juvenile offenders. Child abuse \& Neglect, 2015, 163-173.

Fraenkel, J., \& Wallen, N. (2009). How to design and evaluate research in education(8th ed.). New York, NY: McGraw-Hill.

Frison, E., \& Eggermont, S. (2015). The impact of daily stress on adolescent's depressed mood: The role of social support seeking through Facebook. Computers in Human Behavior,44, 315-325. 
Furr-Holden, C. D. M., Lee, M. H., Milam, A. J., Johnson, R. M., Lee, K. S., \& Ialongo, N. S. (2011). The growth of neighborhood disorder and marijuana use among urban adolescents: A case for policy and environmental interventions. Journal of Studies on Alcohol and Drugs, 72, 371-379.

Gallagher, C.A., and Dobrin, A. 2006. Deaths in juvenile justice residential facilities. Journal of Adolescent Health 38(6):662-668.

Galvan, A., Hare, T., Voss, H., Glover, G., \& Casey, B. J. (2007). Risk-taking and the adolescent brain: Who is at risk? Developmental Science, 10, F8-F14.

Gardner, M., \& Steinberg, L. (2005). Peer influence on risk taking, risk preference, and risky decision making in adolescence and adulthood: An experimental study. Developmental Psychology, 41(4), 625-635.

Geier, C., \& Luna, B. (2009). The maturation of incentive processing and cognitive control. Pharmacology Biochemistry and Behavior, 93, 212-221.

Gibbons, D. C., \& Blake, G. F. (1976). Evaluating the impact of juvenile diversion programs. Crime \& Delinquency, 22, 411-420.

Gil, A.G., Wagner, E.F., \& Tubman, J.G. (2004). Associations between earlyadolescent substance use and subsequent young-adult substance use disorders and psychiatric disorders among a multiethnic male sample in South Florida. American Journal of Public Health, 94, 1603-1609.

Gladwin, T. E., Figner, B., Crone, E. A., \& Wiers, R. W. (2011). Addiction, adolescence, and the integration of control and motivation. Developmental Cognitive Neuroscience, 1(4), 364-376.

Glueck, S., and Glueck, E. 1968. Delinquents and Non-delinquents in Perspective. Cambridge, MA: Harvard University Press.

Gogtay, N., Giedd, J.N., Lusk, L., Hayashi, K.M., Greenstein, D., Vaituzis, A.C., et al. (2004). Dynamic mapping of human cortical development during childhood through early adulthood. Proceedings of the National Academy of Sciences of the United States of America, 101, 8174-9.

Goldstrom, I., Jaiquan, F., Henderson, M., Male, A., \& Manderscheid, R.W. (2000). The availability of mental health services to young people in juvenile justice facilities: A national survey. In R.W Manderscheid \& M.J Henderson (Eds.), Mental Health, United States, 2000. Washington, DC: U.S Government Printing Office.

Greenwood, P. (2008). Prevention and intervention programs for juvenile offenders. 
The Future of Children, 18(2).

Geier, C. F., \& Luna, B. (2009). The maturation of incentive processing and cognitive control. Pharmacology Biochemistry and Behavior, 93, 212-221.

Greydanus, D., \& Patel, D. (2005). The adolescent and substance abuse: Current concepts. Current Problems in Pediatric and Adolescent Health Care, 35, 78-98.

Guttmannova, K., Bailey, J., Hill, K., Lee, J., Hawkins, J., Woods, M., \& Catalano, R. (2011). Sensitive periods for adolescent alcohol use initiation: Predicting the lifetime occurrence and chronicity of alcohol problems in adulthood. Journal of Studies on Alcohol and Drugs,72, 221-231.

Hall JA, Valente TW. (2007). Adolescent smoking networks: The effects of influence and selection on future smoking. Addictive Behaviors. 32:3054-3059.

Hampton, A. S., Drabick, D. A., \& Steinberg, L. (2014). Does IQ moderate the relation between psychopathy and juvenile offending. Law and Human Behavior, 38(1), 23-33.

Handley, E. D., Rogosch, F. A., Guild, D. J., \& Cicchetti, D. (2015). Neighborhood disadvantage and adolescent substance use disorder: The moderating role of maltreatment. Child Maltreatment, 1-10.

Hansell, S., White, H. R. (1991). Adolescent drug use, psychological distress, and physical symptoms. Journal of Health and Social Behavior. 32: 288-301.

Hanson, K., Medina, K., Padula, C., Tapert, S., \& Brown, S. (2011). Impact of adolescent alcohol and drug use on neuropsychological functioning in young adulthood: 10-Year outcomes. Journal of Child and Adolescent Substance Abuse, 20, 135-154.

Hart, Timothy C. \& Waller, Jeremy. 2013. "Neighborhood Boundaries and Structural Determinants of Social Disorganization: Examining the Validity of Commonly Used Measures." Western Criminology Review 14(3): 16-33.

Hawkins, J.D., and Weis, J.G. 1985. The social development model: An integrated approach to delinquency prevention. Journal of Primary Prevention 6:37-97.

Hawkins, J.D., Catalano R.F., Arthur, M.W (2002). Promoting science-based prevention in communities. Addictive Behaviors, 27 (6), 951-976.

Hayes, A. F. (2012). PROCESS: A versatile computational tool for observed variable mediation, moderation, and conditional process modeling [White paper]. Retrieved from http://www.afhayes.com/ public/process2012.pdf 
Haynes R, Daras K, Reading R, Jones A. (2007). Modifiable neighborhood units, zone design and residents' perceptions. Health Place, 13 (4), 812-825.

Henry, K. L., Knight, K. E., \& Thornberry, T. P. (2012). School disengagement as a predictor of dropout, delinquency, and problem substance use during adolescence and early adulthood. Journal of Youth and Adolescence, 41, 156-166.

Henggeler, S. W., \& Schoenwald, S. K. (2011). Evidence-based interventions for juvenile offenders and juvenile justice policies that support them. Social Policy Report, 25(1), 1-27.

Herrenkohl, T. I., Lee, J., \& Hawkins, J. D. (2012). Risks versus direct protective factors and youth violence Seattle Social Development Project. American Journal of Preventative Medicine, 43(21), S41-S56.

Herrenkohl, T. I., Tajima, E. A., Whitney, S. D., \& Bu Huang, M. A. (2005). Protection against antisocial behavior in children exposed to physically abusive discipline. Journal of Adolescent Health, 36(6), 457-465.

Hockenberry, S., and Puzzanchera, C.. 2014. Juvenile Court Statistics 2011. Pittsburgh, PA: National Center for Juvenile Justice.

Hoeve, M., McReynold, L., \& Wasserman, G. (2014). Service referral for juvenile justice youths: Associated with psychiatric disorder and recidivism. Administration and Policy in Mental Health and Mental Health Services Research, 41(3), 379-389.

Hoeve, M., McReynolds, L., Wasserman, G., \& McMillian, C. (2013). The influence of mental health disorders on severity of reoffending in juveniles. Criminal Justice and Behavior, 40(3), 289-301.

Hoeve, M., Dubas, J. S., Eichelsheim, V. I., van der Laan, P. H., Smeenk, W., \& Gerris, J. R. M. (2009). The relationship between parenting and delinquency: A metaanalysis. Journal of Abnormal Child Psychology, 37, 749-775.

Hoge, R. D., Andrews, D. A., \& Leschied, A. W. (1996). An investigation of risk and protective factors in a sample of youthful offenders. Journal of Child Psychology and Psychiatry,37(4), 419-424.

Hummel, A., Shelton, K. H., Heron, J., Moore, L., \& Van den Bree, M. B. (2012). A systematic review of the relationship between family functioning, pubertal timing and adolescent substance use. Addiction, 108, 487-496.

Huttenlocher, P.R., Dabholkar, A.S. (2008). Regional differences in synapto-genesis in human cerebral cortex. Journal of Neuroscience, 387, 167-178. 
Jacobus, J., Bava, S., Cohen-Zion, M., Mahmood, O., \& Tapert, S. F. (2009). Functional Consequences of Marijuana Use in Adolescents. Pharmacology, Biochemistry, and Behavior, 92(4), 559-565.

Johnston, L. D., O’Malley, P. M., Miech, R. A., Bachman, J. G., \& Schulenberg, J. E. ( (2016). Monitoring the Future national survey results on drug use, 1975-2015: Overview, key findings on adolescent drug use. Ann Arbor: Institute for Social Research, The University of Michigan.

Jones, A. M. (2015). When in Rome: Testing the moderating influence of neighborhood composition on the relationship between self-control and juvenile offending. Crime \& Delinquency, 1-27.

Joshi, P.T. (2014). Mental Health Services for Children and Adolescents: Challenges and Opportunities. JAMA Psychiatry, 71(1), 17-18.

Kandel, D.B., Raveis, V.H., Davies, M. (1991). Suicidal ideation in adolescence: Depression, substance use and other factors. Journal of Youth and Adolescence, 20(2), 289-309.

Kandel, E., Mednick, S. A., Kirkegaard-Sorensen, L., Hutchings, B., Knop, J., Rosenberg, R., \& Schulsinger, F. (1988). IQ as a protective factor for subjects at high risk for antisocial behavior. Journal of Consulting and Clinical Psychology, 56(2), 224-226.

Katon, W., Richardson, L., Russo, J., McCarty, C. A., Rockhill, C., McCauley, E., ... Grossman, D. C. (2010). Depressive symptoms in adolescence: the association with multiple health risk behaviors. General Hospital Psychiatry, 32(3), 233-239.

Kazdin, A. (2003). Adolescent development, mental disorders, and decision making of delinquency youths. In T. Grisso \& R.Schwartz (Eds.), Youth on trial: A developmental perspective on juvenile justice. Chicago: University of Chicago Press.

Kelder, S. H., Murray, N. G., Orpinas, P., Prokhorov, A., McReynolds, L., Zhang, Q., $\&$ Roberts, R. (2001). Depression and substance use in minority middle-school students.American Journal of Public Health, 91(5), 761-766.

Kellam, S. G., Ling, X., Merisca, R., Brown, C. H., \& Ialongo, N. (1998). The effect of the level of aggression in the first grade classroom on the course of malleability of aggressive behavior in $\mathrm{mi}$

Kerig, P. K., \& Becker, S. P. (2014). 12 early abuse and neglect as risk factors for the development of criminal and antisocial behaviors. The Development of Criminal and Antisocial Behavior, 181-199. 
Kessler, C. (2002). Need for attention to mental health of young offenders. The Lancet, $359,1956-1957$.

King, K. M., \& Chassin, L. (2007). A prospective study of the effects of age of initiation of alcohol and drug use on young adolescent substance dependence. Journal of Studies on Alcohol and Drugs, 68, 256-265.

King, K. M., \& Chassin, L. (2004). Mediating and moderated effects of adolescent behavioral undercontrol and parenting in the prediction of drug use disorders in emerging adulthood. Psychology of Addictive Behaviors, 18, 239-249

Kingston, B., D. H., \& Elliot, D. S. (2009). A test of social disorganization theory in high risk urban neighborhoods. Youth \& Society, 1-27.

Konrad, K., Firk, C., \& Uhlhaas, P. (2013). Brain development during adolescence. Deutsches Arzteblatt International, 110(25), 425-431.

Kretschmar, J., Butcher, F., Flannery, D., \& Singer, M. (2014). Diverting juvenile justice-involved youth with behavioral health issues from detention: Preliminary findings from Ohio's Behavioral Health Juvenile Justice (BHJJ) Initiative. Criminal Justice Policy Review, 1-24.

Kretschmar, J.M., Butcher, F., \& Flannery, D. (2013). An evaluation of the behavioral health/juvenile justice initiative. Behavioral Health in Ohio-Current Research Trends, 1(2), 18-30.

Kroneman, L., Loeber, R., \& Hipwell, A. (2004). Is neighborhood context differently related to externalizing problems and delinquency for girls compared with boys? Clinical Child and Family Psychology Review, 7(2), 109-122.

Lambert, S. F., Brown, T. L., Phillips, C. M., \& Ialongo, N. S. (2004). The relationship between perceptions of neighborhood characteristics and substance use among urban African American adolescents. American Journal of Community Psychology, 34(3/4), 205-218.

Lambie, I., \& Randell, I. (2013). The impact of incarceration on juvenile offenders. Clinical Psychology Review, 33(3), 448-459.

Langford, C.P.H.; Bowsher, J.; Maloney, J.P.; Lillis, P.P. (1997). "Social support: a conceptual analysis". Journal of Advanced Nursing 25: 95-100.

Lansford, J. E., Miller-Johnson, S., Berlin, L. J., Dodge, K. A., Bates, J. E., \& Pettit, G. S. (2007). Early physical abuse and later violent delinquency: A prospective longitudinal study. Child Maltreatment, 12(3), 233-245.

Lansing, A. E., Washburn, J. J., Abram, K. M., Thomas, U. C., Welty, L. J., \& Teplin, 
L. A. (2014). Cognitive and academic functioning of juvenile detainees: Implications for correctional populations and public health. Journal of Correctional Health Care, 20(1), 18-30.

Lazarus, R., \& Folkman, S. (1984). Stress, Appraisal, and Coping. New York: Springer.

Leventhal, T., \& Brooks-Gunn, J. (2000). The neighborhoods they live in: The effects of neighborhood residence on child and adolescent outcomes. Psychological Bulletin, 126(2), 309-337.

Levine, S. B., \& Coupey, S. M. (2003). Adolescent substance use, sexual behavior and metropolitan status: is "urban" a risk factor? Journal of Adolescent Health, 32(5), 350-355.

Liebenberg, L., \& Unger, M. (2014). A comparison of service use among youth involved with juvenile justice and mental health. Children and Youth Services Review, 39, 117-122.

Lipton, D., Martinson, R., \& Wilks, J. (1975). The effectiveness of correctional treatment: A survey of treatment evaluation studies. New York: Praeger.

Lo, C. C., Webar, J., \& Cheng, T. C. (2015). Community's role and school's role in protecting against substance use: A spatial analysis. Journal of Child \& Adolescent Substance Abuse, 24(4), 198-206.

Lobiondo-Wood, G. (2002). Introduction to quantitative research. In G. LobiondoWood \& J. Haber (Eds.), Nursing research: methods, critical appraisal, and utilization (5th ed., pp.187-201). St. Louis: Mosby.

Loeber, R. (1990). Development and risk factors of juvenile anti-social behavior and delinquency. Clinical Psychology Review, 10, 1-41.

Losel, F., \& Farrington, D. P. (2012). Direct protective and buffering protective factors in the development of youth violence. American Journal of Preventative Medicine, 43(2), S8-S23.

Luna, B., Paulsen, D., Padmanabhan, A., \& Geier, C. (2013). The Teenage Brain: Cognitive Control and Motivation. Current Directions in Psychological Science, 22(2), 94-100.

Macintyre S, Ellaway A. (2003). Neighborhoods and health: Neighborhoods and Health. New York: Oxford University Press; 20-42.

MacDonald, K. B. (2008). Effortful control, explicit processing, and the regulation of human evolved predispositions. Psychological Review, 115(4), 1012-1031. 
Mandelbaum, D. E., \& De la Monte, S. M. (2017). Adverse structural and functional effect of marijuana on the brain: Evidence reviewed. Pediatric Neurology, 66, 1220.

Massey, D., Gross, A., Eggers., M. (1991). Segregation, the concentration of poverty, and the life chances of individuals. Social Science Research, 20, 397-420.

Massey, D., \& Hajnal., Z. (1995). The changing geographic structure of Black-White segregation in the United States. Social Science Quarterly,76: 527-542.

Masten, A. S. (2007). Resilience in developing systems: Progress and promise as the fourth wave rises. Development and Psychopathology, 19, 921-930.

Masten, A. S., Best, K. M., \& Garmezy, N. (1990). Resilience and development: Contributions from the study of children who overcome adversity. Development and Psychopathology, 2, 425-444.

Masten, A. S., \& Coatsworth, J. D. (1998). The development of competence in favorable and unfavorable environments: Lessons from successful children. American Psychologist, 53, 205-220.

Matthews, S. K., Krivelyova, A., Stephens, R. L., \& Bilchik, S. (2011). Juvenile justice contact of youth in systems of care: Comparison study results. Criminal Justice Policy Review,24(2), 143-165.

Maxfield, M. G., \& Widom, C. S. (1996). The cycle of violence revisited 6 years later. Archives of Pediatrics and Adolescent Medicine, 150, 390-395.

McCarty, C. A., Stoep, A. V., Kuo, E. S., \& McCauley, E. (2006). Depressive symptoms among delinquent youth: Testing models of association with stress and support. Journal of Psychopathology and Behavioral Assessment, 28(2), 85-93.

McCormick, S., Peterson-Badali, M., \& Skilling, T. A. (2015). Mental health and justice system involvement: A conceptual analysis of the literature. Psychology, Public Policy and Law, 21(2), 213-225.

McGrath, B., Brennan, M., Dolan, P., \& Barnett, R. (2012). Adolescents and their networks of social support: Real connections in real lives? Child and Family Social Work; 19, 237-248.

McGrath, J., Welham, J., Scott, J., Varghese, D., Degenhardt, L., Hayatbakhsh, M. R., Najman, J. M. (2010). Association between cannabis use and psychosis-related outcomes using sibling pair analysis in a cohort of young adults. Archives of General Psychiatry, 67(5), 440-447. 
McKenzie, M., Olsson, C. A., Jorm, A. F., Romaniuk, H., \& Patton, G. C. (2010). Association of adolescent symptoms of depression and anxiety with daily smoking and nicotine dependence in young adulthood: Findings from a 10 year longitudinal study. Addiction,105, 1652-1659.

Medina, K. L., Hanson, K. L., Schweinsburg, A. D., Cohen-Zion, M., Nagel, B. J., \& Tapert, S. F. (2007). Neuropsychological functioning in adolescent marijuana users: Subtle deficits detectable after a month of abstinence. Journal of the International Neuropsychological Society, 13, 807-820.

Mendel, R. A. (2011). No place for kids: The case for reducing juvenile incarceration. Baltimore, MD: Annie E. Casey Foundation.

Mental Illness exacts heavy toll, beginning in youth. (2005, January 1). Retrieved February 1, 2015, from http://www.nimh.nih.gov/news/sciencenews/2005/mental-illness-exacts-heavy-toll-beginning-in-youth.shtml

Mertler, C. A. \& Vannatta, R. A. (2005). Advanced and multivariate statistical methods: practical application and interpretation (3rd ed.). Glendale, CA: Pyrczak Publishing.

Miller, A. K. H.; Alston R.L., Corsellis, J.A. (1980). Variation with age in the volumes of grey and white matter in the cerebral hemisphere of man: measurements with an image analyser. Neuropathology and Applied Neurobiology 6 (2): 119-132

Moffitt, T. E., \& Caspi, A. (2001). Childhood predictors differentiate life-coursepersistent and adolescence-limited antisocial pathways among males and females. Development and Psychopathology, 13, 355-375.

Moffitt, T. E., Caspi, A., Dickson, N., Silva, P., \& Stanton, W. (1996). Childhood-onset versus adolescent-onset antisocial conduct problems in males: Natural history from ages 3 to 18 years. Development and Psychopathology, 8(2), 399-424.

Molnar, B. E., Cerda, M., Roberts, A. L., \& Burk, S. L. (2008). Effects of neighborhood resources on aggressive and delinquent behaviors among urban youth. American Journal of Public Health, 98(6), 1086-1093.

Monahan, K., Rhew, I., Hawkins, D., \& Brown, E. (2013). Adolescent pathways to cooccurring problem behavior: The effects of peer delinquency and peer substance use. Journal of Research on Adolescence,24(4), 630-645.

Moody, J., Brynilden, W. D., Osgood, D. W., Feinberg, M. E., \& Gest, S. (2011). Popularity trajectories and substance use in early adolescence. Social Networks, $33,101-112$.

Moretti, E. (2005). Does education reduce participation in criminal activities? In H. M. 
Levin (Chair), Symposium on the social costs of inadequate education conducted at Teachers College. New York: Columbia University.

Moss, H. B., Chen, C. M., \& Yi, H. Y. (2014). Early adolescent patterns of alcohol, cigarettes, and marijuana polysubstance use and young adult substance use outcomes in a nationally representative sample. Drug and Alcohol Dependence, $136,51-62$.

Most Commonly Used Addictive Drugs | National Institute on Drug Abuse (NIDA). (2014). Retrieved from http://www.drugabuse.gov/publications/mediaguide/most-commonly-used-addictive-drugs

Muncie, J. (2009). The United Nations, children's rights and juvenile justice. In W. Taylor, R. Earle, \& R. Hester (Eds.), Youth justice handbook: Theory, policy and practice.

Murray, J., Irving, B., Farrington, D. P., Colman, I., \& Bloxsom, C. A. J. (2010). Very early predictors of conduct problems and crime: Results from a national cohort study. Journal of Child Psychology and Psychiatry, 51, 1198-1207.

Nakagawa, A., \& Sukigara, M. (2012). Difficulty in disengaging from threat and temperamental negative affectivity in early life: A longitudinal study of infants aged 12-36 months. Behavioral and Brain Functions, 8(1), 1-8.

Nansel, T. R., Overpeck, M. D., Haynie, D. L., Ruan, J., \& Scheidt, P. C. (2003). Relationships between bullying and violence among US youth. Archives of Pediatrics and Adolescent Medicine, 157, 348-353.

National Institute on Drug Abuse. (2014). The Science of Drug Abuse \& Addiction. Retrieved from http://www.drugabuse.gov

Nilsen, W., Karevold, E., Roysamb, E., Gustavson, K., \& Mathiesen, K. (2013). Social skills and depressive symptoms across adolescence: Social support as a mediator in girls versus boys. Journal of Adolescence, 36, 11-20.

Nkyi, A. K. (2015). Adolescent use of alcohol, tobacco and marijuana: The gateway to other drugs. International Journal of Psychology and Behavioral Sciences, 5(4), 158-168.

Nisar, M., Ullah, S., Ali, M., \& Alam, S. (2015). Juvenile delinquency: The influence of family, peer and economic factors on juvenile delinquents. Applied Science Reports, 9(1), 37-48.

Ogles, B. M., Melendez, G., Davis, D. C., \& Lunnen, K. M. (2001). The Ohio Scales: Practical outcome assessment. Journal of Child and Family Studies, 10(2), 199212. 
Ohio Mental Health and Addiction Services: Promoting Wellness and Recovery. (n.d). Retrieved June 15, 2015, from http://mha.ohio.gov

O’Leary CM. (2004). Fetal alcohol syndrome: diagnosis, epidemiology, and developmental outcomes. Journal of Paediatric and Child Health 40:2-7.

Olds D. (1997). Tobacco exposure and impaired development: a review of the evidence. Mental Retardation and Developmental Disabilities Research Review. 3:257-269.

Osgood, D. W. \& Chambers, J. M. (2000). Social disorganization outside the metropolis: An analysis of rural youth violence. Criminology, 38(1), 81-116.

Pardini, D., White, H., Xiong, S., Bechtold, J., Chung, T., Loeber, R., \& Hipwell, A. (2015). Unfazed or Dazed and Confused: Does Early Adolescent Marijuana Use Cause Sustained Impairments in Attention and Academic Functioning? Journal of Abnormal Child Psychology, 43(7), 1203-1217.

Parker JS \& Benson MJ. (2004). Parent-adolescent relations and adolescent functioning: Self-esteem, substance abuse, and delinquency. Adolescence, 39(155):519-530.

Patrick, M. E., \& Schulenberg, J. E. (2014). Prevalence and predictors of adolescent alcohol use and binge drinking in the United States. Alcohol Research: Current Reviews, 35(2), 193-200.

Patrick, M., \& Schulenberg, J.E. (2011) How trajectories of reasons for alcohol use relate to trajectories of binge drinking: national panel data spanning late adolescence to early adulthood. Developmental Psychology 47(2):311-317.

Patrick, M., Schulenberg, J., O'malley, P., Johnston, L., \& Bachman, J. (2011). Adolescents' reported reasons for alcohol and marijuana use as predictors of substance use and problems in adulthood. Journal of Studies on Alcohol and Drugs, 72, 106-116.

Patten C.A, Gillin C.J, Farkas A.J, Gilpin E.A, Berry C.C, \& Pierce J.P. (1997). Depressive symptoms in California adolescents: Family structure and parental support. Journal of Adolescent Health. 20:271-278.

Paus, T. (2005). Mapping brain maturation and cognitive development during adolescence. Trends in Cognitive Sciences, 9, 60-8.

Paxton, R. J., Valois, R. F., Watkins, K. W., Huebner, E. S., \& Drane, J. W. (2007). Associations between depressed mood and clusters of health risk behaviors. American Journal of Health Behaviors, 31(3), 272-283. 
Pedhazur, E. J. (1982). Multiple regression in behavioral research (2nd ed.). New York: Harcourt Brace College Publishers.

Penney, S.R., and Moretti, M.M. 2005. The transfer of juveniles to adult court in Canada and the United States: Confused agendas and compromised assessment procedures. International Journal of Forensic Mental Health 4(1):19-37.

Perrin J. S, Leonard G., Perron M., Pike G. B, Pitiot A., Richer L., Veillette S., Pausova Z., Paus T. (2008): Growth of white matter in the adolescent brain: Role of testosterone and androgen receptor. Journal of Neuroscience 28:9519-9524.

Perrin, J., Herve, P., Leonard, G., Perron, M., Pike, G., Pitiot, A., Paus, T. (2008). Growth of white matter in the adolescent brain: Role of testosterone and androgen receptor. The Journal of Neuroscience, 28(38), 9519-9524.

Petitclerc, A., Gatti, U., Vitaro, F., \& Tremblay, R. E. (2013). Effects of juvenile court exposure on crime in young adulthood. Journal of Child Psychology and Psychiatry, 54(3), 291-297.

Petraitis, J., Flay, B., \& Miller, T. (1995). Reviewing theories of adolescent substance use: Organizing pieces in the puzzle. Psychological Bulletin, 117(1), 67-86.

Pham-Kanter, Genevieve. (2001). "Substance abuse and dependence." The Gale Encyclopedia of Medicine. Second Edition. Jacqueline L. Longe, Ed. 5 vols. Farmington Hills, MI: Gale Group.

Phillippi, S. W., Cocozza, J., \& Deprato, D. K. (2013). Advancing evidence-based practices for juvenile justice reform through community development. Journal of Community Practice, 21(4), 434-450.

Physical Activity has many Health Benefits. (n.d.). Retrieved January 7, 2015, from http://www.health.gov/paguidelines/guidelines/Chapter2.aspx

Pickett KE, Pearl M. (2001) Multilevel analyses of neighborhood socioeconomic context and health outcomes: a critical review. Journal of Epidemiology and Community Health. 55(2):111-122.

Pinto, H., R., Logsdon, M.C., Burant, C. (2012). Psychometric evaluation of the revised attribution questionnaire to measure mental illness stigma in adolescents. Journal of Nursing Measurement, 20(1), 47-58.

Portnoy, J., Chen, F. R., \& Raine, A. (2013). Biological protective factors for antisocial and criminal behavior. Journal of Criminal Justice, 41(5), 292-299.

Post, M. W. M. (2014). Definitions of Quality of Life: What Has Happened and How to 
Move On. Topics in Spinal Cord Injury Rehabilitation, 20(3), 167-180. http://doi.org/10.1310/sci2003-167

Preamble to the Constitution of the World Health Organization as adopted by the International Health Conference, New York, 19-22 June, 1946; signed on 22 July 1946 by the representatives of 61 States (Official Records of the World Health Organization, no. 2, p. 100) and entered into force on 7 April 1948.

Puzzanchera,C. (2009). Juvenile arrests 2007. Washington, DC: U.S. Department of Justice, Office of Juvenile Justice and Delinquency Prevention.

Ragan, D. T., Osgood, D. W., \& Feinberg, M. E. (2013). Friends as a bridge to parental influence: Implications for adolescent alcohol use. Social Forces, 1-25.

Ramo, D., Liu, H., \& Prochaska, J. (2012). Tobacco and marijuana use among adolescents and young adults: A systematic review of their co-use. Clinical Psychology Review, 32, 105-121.

Reboussin, B. A., Green, K. M., Milam, A. J., Furr-Holden, C. D., \& Ialongo, N. S. (2014). Neighborhood environment and urban African American marijuana use during high school. Journal of Urban Health: Bulletin of the New York Academy of Medicine, 91(6), 1189-1201.

Redonnet, B., Chollet, A., Fombonne, E., Bowes, L., \& Melchior, M. (2012). Tobacco, alcohol, cannabis and other illegal drug use among young adults: The socioeconomic context. Drug and Alcohol Dependence, 121, 231-239.

Reitz-Krueger, C. L., Nagel, A. G., Guarnrea, L. A., \& Dickon Reppucci, N. (2014). Community influence on adolescent development. Handbook of Adolescent Behavioral Problems, 71-84.

Reppucci, N. (1999). Adolescent Development and Juvenile Justice. American Journal of Community Psychology, 27(3).

Reynolds, A. D., \& Crea, T. M. (2015). Peer influence processes for youth delinquency and depression. Journal of Adolescence, 43, 83-95.

Robinson M, Oddy W, McLean N, Jacoby P, Pennell C, de Klerk N, Zubrick S, Stanley F, Newnham J. (2010) Low-moderate prenatal alcohol exposure and risk to child behavioural development: a prospective cohort study. BJOG: An International Journal of Obstetrics and Gynecology, 117:1139-1152.

Rubino, T., Zamberletti, E., \& Parolaro, D. (2012). Adolescent exposure to cannabis as a risk factor for psychiatric disorders. Journal of Psychopharmacology, 26(1), $177-188$. 
Rutter, M. (2012). Resilience as a dynamic concept. Development and Psychopathology, 24(2), 335-344.

Rutter, M. (1987). Psychosocial resilience and protective mechanisms. American Journal of Orthopsychiatry 57(3):316-331.

Rutter, M., \& Quinton, D. (1984) Long-term follow-up of women institutionalized in childhood-factors promoting good functioning in adult life. British Journal of Developmental Psychology, 2, 191-204.

Ryan, E. P., \& Redding, R. E. (2004). A review of mood disorders among juvenile offenders. Psychiatric Services, 55(12), 1397-1407.

Sampson, R., \& Morenoff, J. (2004). Spatial (dis)advantage and homicide in Chicago neighborhoods. In M. Goodchild, \& D. Janelle (Eds.), Spatially integrated social science (pp. 145e170).

Sampson R, Morenoff J, Gannon-Rowley T. (2002) Assessing "neighborhood effects": Social processes and new directions in research. Annual Review of Sociology. 28, 443-478.

Sampson, R. J. (1997). Collective regulation of adolescent misbehavior: Validation results from eighty Chicago neighborhoods. Journal of Adolescent Research, 12, 227-244.

Sampson, R., Raudenbush, S., \& Earls, F. (1997). Neighborhood and violent crimes: A multilevel study of collective efficacy. Science, 277(15), 918-924.

Sampson, R., \& Groves, B. (1989). Community structure and crime: Testing socialdisorganization theory. American Journal of Sociology, 94(4), 774-802.

Sawyer, S. M., Afifi, R. A., Bearinger, L., Blakemore, S. J., Dick, B., Ezeh, A. C., \& Patton, G. C. (2012). Adolescence: a foundation for future health. Lancet, 379, 1630-1640.

Schonfeld, A. M., Mattson, S. N., \& Riley, E. P. (2005). Moral maturity and delinquency after prenatal alcohol exposure. Journal of Studies on Alcohol, 66, 545-554.

Schubert, C. A., Mulvey, E. P., \& Glasheen, C. (2011). Influence of mental health and substance use problems and criminogenic risk on outcomes in serious juvenile offenders. Journal of the American Academy of Child and Adolescent Psychiatry, 50(9), 925-937.

Schulenberg, J., Patrick, M. E., Maslowsky, J., \& Maggs, J. L. (2014). Handbook of Developmental Psychopathology. New York: Springer Science + Business Media. 
Schwalbe, C. S., Gearing, R. E., Mackenzie, M. J., Brewer, K. B., \& Ibrahim, R. (2012). A meta-analysis of experimental studies of diversion programs for juvenile offenders. Clinical Psychology Review, 32(1), 26-33.

Schwarz, S. W. (2009). Adolescent mental health in the United States: Facts for Policymakers. Retrieved February 5, 2015, from http://nccp.org/publications/pdf/text_878.pdf

Schweinsburg, A. D., Brown, S. A., \& Tapert, S. F. (2008). The Influence of Marijuana Use on Neurocognitive Functioning in Adolescents. Current Drug Abuse Reviews, 1(1), 99-111.

Scott, E., \& Grisso, T. (1997). The Evolution of Adolescence: A developmental perspective on juvenile. The Journal of Criminal Law and Criminology, 88(1), 137-189.

Seroczynski, A. D., Evans, W. N., Jobst, A. D., Horvath, L., \& Carozza, G. (2015). Reading for life and adolescent re-arrest: Evaluating a unique juvenile diversion program. Wilson Sheehan Lab for Economic Opportunities, 1-52.

Shader, M. (2001). Risks factors for delinquency: An overview. Retrieved from http://www.behavioralinstitute.org/uploads/Risk_Factors_for_Delinquency_OJJD P.pdf

Shaw, C. R., \& McKay, H. D. (1942). Juvenile delinquency and urban areas. Chicago: University of Chicago Press.

Siegel, L. J.\& Welsh, B.(2011). Juvenile Delinquency: The Core (4th ed.). Belmont, CA: Wadsworth/cengage Learning.

Simantov E, Schoen C, Klein JD. (2006). Health-compromising behaviors: Why do adolescents smoke or drink? Archives of Pediatrics and Adolescent Medicine, 154:1025-1033.

Skowyra, K.R., and Cocozza, J.J (2007). Blueprint for Change: A Comprehensive Model for the Identification and Treatment of Youth with Mental Health Needs in Contact with the Juvenile Justice System. Delmar, NY: National Center for Mental Health and Juvenile Justice, Policy Research Associates, Inc.

Skowya, K., \& Cocozza, J. J. (2006). A blueprint for change: Improving the system response to youth with mental health needs involved with the juvenile justice system. Retrieved from http://www.ncmhjj.com/wpcontent/uploads/2013/07/2006_A-Blueprint-for-Change.pdf

Skowyra, K. \& Powell, S. (2006). Juvenile diversion: Programs for justice-involved 
youth with mental health disorders. Delmar, NY: National Center for Mental Health and Juvenile Justice.

Slotkin T. (1998) Fetal nicotine or cocaine exposure: which one is worse? Journal of Pharmacology and Experimental Therapeutics. 285: 931-945.

Snedker, K. A., Herting, J. R., \& Walton, E. (2009). Contextual effects and adolescent substance use: Exploring the role of neighborhoods. Social Science Quarterly, 90(5), 1272-1297.

Squeglia, L. M., Jacobus, J., \& Tapert, S. F. (2009). The influence of substance use on adolescent brain development. Clinical EEG and neuroscience, 40(1), 31-38.

Squeglia, L. M., Rinker, D. A., Bartsch, H., Castro, N., Chung, Y., Dale, A. M., Jernigan, T. L. (2014). Brain volume reductions in adolescent heavy drinkers. Developmental Cognitive Neuroscience, 9, 117-125.

Steinberg, L. (2005). Adolescence. 7th ed. Boston, MA: McGraw Hill.

Steketee, M., Junger, M., \& Junger-Tas, J. (2013). Sex differences in predictors of juvenile delinquency: Females are more susceptible to poor environments; males are influenced more by law self-control. Journal of Contemporary Criminal Justice, 29(1), 88-105.

Stone, A., Becker, L., Huber, A., \& Catalano, R. (2012). Review of risk and protective factors of substance use and problem use in emerging adulthood. Addictive Behaviors, 37, 747-775.

Sung, M., Erkanli, A., Angold, A., \& Costello, E. J. (2004). Effects of age at first substance use and psychiatric co-morbidity on the development of substance use disorders. Drug and Alcohol Dependence, 75, 287-299.

Tabachnick, B. G. \& Fidell, L. S. (2007). Using multivariate statistics (5th ed.). Boston: Pearson.

Teplin, L. Abram, K.M., Washburn J.J, Welty, L.J. Hershfield J.A., and Dulcan, M.K (2013). The Northwestern Juvenile Project: Overview. U.S. Department of Justice.

Teplin, L. A., Elkington, K. S., McClelland, G. M., Abram, K. M., Mericle, A. A., \& Washburn, J. J. (2005). Major mental disorders, substance use disorders, comorbidity, and HIV-AIDS risk behaviors in juvenile detainees. Psychiatric Services, 56(7), 823-828.

Tomey A.M, Alligood. MR. Nursing theorists and their work. (5th ed.). Mosby, Philadelphia, 2002. 
Tompsett, C. J., Amrhein, K. E., \& Hassan, S. (2014). Travel beyond the home neighborhood for delinquent behaviors: Moderation of home neighborhood influence. Journal of Adolescence, 37(4), 325-333.

Trucco, E. M., Colder, C. R., Wieczorek, W. F., Lengua, L. J., \& Hawk Jr., L. W. (2014). Early adolescent alcohol use in context: How neighborhoods, parents and peers impact youth. Developmental Psychopathology, 26(2), 425-436.

Tucker, J. S., Pollard, M. S., De la Haye, K., Kennedy, D. P., \& Green, H. D. (2013). Neighborhood characteristics and the initiation of marijuana and binge drinking. Drug and alcohol dependence, 128(1-2), 83-89.

Turner, M. G., Hartman, J. H., \& Bishop, D. M. (2007). The effects of prenatal problems, family functioning, and neighborhood disadvantage in predicting lifecourse-persistent offending. Criminal Justice and Behavior, 34(10), 1241-1261.

Turner, R. J., \& Marino, F. (1994). Social support and social structure: A descriptive epidemiology. Journal of Health and Social Behavior, 35, 193-212.

Unruh D, Povenmire-Kirk T, Yamamoto S. (2009) Perceived barriers and protective factors of juvenile offenders on their developmental pathway to adulthood. Journal of Correctional Education, 60(3):201-225.

U.S. Department of Health and Human Services. The Health Consequences of Smoking - 50 Years of Progress: A Report of the Surgeon General. Atlanta, GA: U.S. Department of Health and Human Services, Centers for Disease Control and Prevention, National Center for Chronic Disease Prevention and Health Promotion, Office on Smoking and Health, 2014. http://www.surgeongeneral.gov/library/reports/50-years-of-progress/fullreport.pdf.

Vakalahi, H. (2001). Adolescent substance use and family-based risk and protective factors: A literature review. Journal of Drug Education, 31(1), 29-46.

Vanderbilt-Adriance, E., \& Shaw, D. S. (2008). Protective factors and the development of resilience in the context of neighborhood disadvantage. Journal of Abnormal Child Psychology, 36, 887-901.

Vaux, A. (1985). Variations in social support associated with gender, ethnicity and age. Journal of Social Issues, 41(1), 89-110.

Viner, R. M., Ozer, E. M., Denny, S., Marmot, M., Resnick, M., Fatusi, A., \& Currie, C. (2012). Adolescence and the social determinants of health. Lancet, 379, 16411652 . 
Vogel, M., Rees, C. E., McCuddy, T., \& Carson, D. C. (2015). The Highs That Bind: School Context, Social Status and Marijuana Use. Journal of Youth and Adolescence, 44, 1153-1164.

Vogel, M., \& Barton, M. (2013). Impulsivity, school context, and school misconduct. Youth \& Society, 45(4), 455-479.

Volkow, N. D., Baler, R. D., Compton, W. M., \& Weiss, S. R. (2014). Adverse health effects of marijuana use. The New England Journal of Medicine, 370(23), 22192227.

Von Ah, D., Ebert, S., Ngamvitroj, A., Park, N., \& Kang, D. (2005). Factors related to cigarette smoking initiation and use among college students. Tobacco Induced Diseases, 3(1), 1-27.

Vuolo, M., \& Staff, J. (2013). Parent and child cigarette use: A longitudinal, multigenerational study. Pediatrics, 132(3), 568-577.

Wagner, K. D., Ritt-Olson, A., Chou, C., Pokhrel, P., Duan, L., Baezconde-Garbanati, L. Soto, D. (2010). Associations between family structure, family functioning and substance use among Hispanic/Latino Adolescents. Psychology of Addictive Behaviors, 24(1), 98-108.

Wakschlag, L. S., Pickett, K. E., Cook, E., Benowitz, N. L., \& Leventhan, B. L. (2002). Maternal smoking during pregnancy and severe anti-social behavior in offspring: A review. American Journal of Public Health, 92(6), 966-974.

Washburn, J.J., Teplin, L.A., Voss, L.S., Simon, C.D., Abram, K.M., and McClelland, G.M (2008) Psychiatric disorders among detained youths: A comparison of youths processed in juvenile court and adult criminal court. Psychiatric Services 59:965-973.

Wasserman, G. A., McReynolds, L. S., Whited, A. L., Keating, J. M., Musabegovic, H., \& Huo, Y. (2008). Juvenile probation officers' mental health decision making. Administration and Policy in Mental Health and Mental Health Services Research, 35(5), 410-422.

Way, N., Stauber, H., Nakkula, M. J., \& London, P. (1994). Depression and substance use in two divergent high school cultures: A quantitative and qualitative analysis. Journal of Youth and Adolescence, 23(3), 331-357.

Weerman, F. M., Harland, P., \& van der Laan, P. H. (2007). Misbehavior at school and delinquency elsewhere: A complex relationship. Criminal Justice Review, 32(4), 358-379.

White, L. M., Aalsma, M. C., Monahan, P. O., \& Pekins, A. (2014). Adolescents 
involved in the juvenile justice system: Epidemiologic study of trends from 19992011. Journal of Adolescent Health, 54(2), s43-s44.

Whitted, K. S., Delavega, E., \& Lennon-Dearing, R. (2013). The youngest victims of violence: Examining the mental health needs of young children who are involved in the child welfare and juvenile justice systems. Child and Adolescent Social Work Journal, 30, 181-195.

Wikström P-OH, Loeber R. (2000) Do disadvantaged neighborhoods cause welladjusted children to become adolescent delinquents? A study of male juvenile serious offending, individual risk and protective factors, and neighborhood context. Criminology, 38(4):1109 - 1142.

Wilcox, H. C., \& Anthony, J. C. (2004). The development of suicide ideation and attempts: An epidemiologic study of first graders followed into young adulthood. Drug and Alcohol Dependence, 7, S53-S67.

Wills, T., Resko, J. A., Ainette, M. G., \& Mendoza, D. (2004). Role of parent support and peer support in adolescent substance use: A test of mediated effects. Psychology of Addictive Behaviors, 18(2), 122-134.

Wilson, H. A., \& Hoge, R. D. (2013). The effect of youth diversion programs on recidivism: A meta-analytic review. Criminal Justice and Behavior, 40(5), 497518.

Wilson, H. A., \& Hoge, R. D. (2012). Diverting our attention to what works: Evaluating the effectiveness of a youth diversion program. Youth Violence and Juvenile Justice, 11(4), 313-331.

Wilson, N., Syme, S., Boyce, W., Battistich, V. A., \& Selvin, S. (2005). Adolescent alcohol, tobacco and marijuana use: The influence of neighborhood disorder and hope. American Journal of Health Promotion, 20(1), 11-19.

World Health Organization (WHO). (2014). Health for the World's Adolescents: A second change in the second decade. Geneva, Switzerland.

What is nursing? (n.d.). Retrieved February 2, 2015, from http://www.nursingworld.org/EspeciallyForYou/What-is-Nursing

Wright, E. M., Fagan, A. A., \& Pinchevsky, G. M. (2014). Penny for your thoughts? The protective effect of youth's attitudes against drug use in high risk communities. Youth Violence and Juvenile Justice, 1-20.

Yen I, Syme SL. (1999) The social environment and health: A discussion of the epidemiologic literature. Annual Review of Public Health. 20:287- 308. 
Zajac, K., Sheidow, A. J., \& Davis, M. (2015). Juvenile justice, mental health, and the transition to adulthood: A review of service system involvement and the unmet needs in the U.S. Children and Youth Services Review, 56, 139-148.

Zimmer-Gembeck, M. J., \& Skinner, E. A. (2011). Review: The development of coping across childhood and adolescence: An integrative review and critique of research. International Journal of Behavioral Development, 35(1), 1 